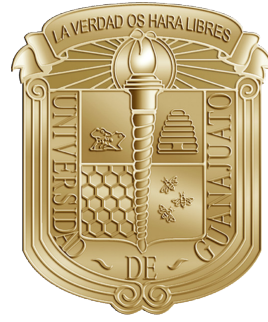

UNIVERSIDAD DE GUANAJUATO

División de ingenierías

Campus Irapuato - Salamanca

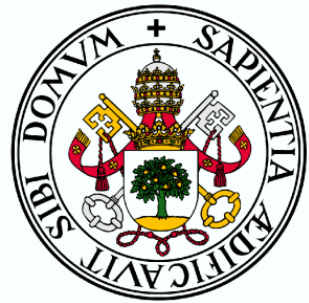

UNIVERSIDAD DE VALLADOLID

Escuela de ingenierías industriales

\title{
Diagnóstico de motores eléctricos para la localización de fallas incipientes
}

\section{TESIS}

En el formato de doble titulación para obtener el grado de:

\section{DOCTOR}

EN INGENIERÍA ELÉCTRICA

por la Universidad de Guanajuato
EN INGENIERÍA INDUSTRIAL por la Universidad de Valladolid

Presenta:

Paulo Antonio Delgado Arredondo

Directores:

Dr. René de Jesús Romero Troncoso

Dr. Daniel Morínigo Sotelo 


\section{Agradecimientos}

\section{A mi esposa:}

Jannet Diosdado G. que ha estado conmigo durante todo este tiempo apoyándome incondicionalmente, gracias por ser como eres y por tu ayuda en esos momentos difíciles.

\section{A mis Padres:}

J. Antonio Jorge Delgado Martinez y Celia Arredondo Armenta por apoyarme durante toda mi vida en todo lo que he hecho, gracias.

\section{A mis Hermanos:}

Juan, Miguel y Ana por todo el apoyo que me han brindado y ser tan buenos hermanos conmigo.

\section{A mis Abuelos:}

Hermilo Arredondo, Enedina Armenta y Carmen Martínez que siempre me han cuidado y se han preocupado por mi. 


\section{A Dr. René de Jesús Romero Troncoso y Dr. Daniel Moríñigo Sotelo:}

Por asesorarme a lo largo del doctorado y acompañarme en este camino que hoy culmina

con la presentación de este proyecto, por compartir su conocimiento conmigo e inspirar en mi mucha admiración. Gracias por todo.

\section{A mis compañeros de la Universidad:}

Gracias por ayudarme y estar conmigo a lo largo del doctorado. 


\section{Agradecimientos institucionales.}

A la Universidad de Guanajuato, y a la División de Ingenierías Campus Irapuato-Salamanca, por permitirme realizar mis estudios de Doctorado. Les agradezco el haberme proporcionado el equipo y las instalaciones necesarias para terminar mis estudios y por hacerme sentir parte de esta gran institución como lo es la Universidad de Guanajuato.

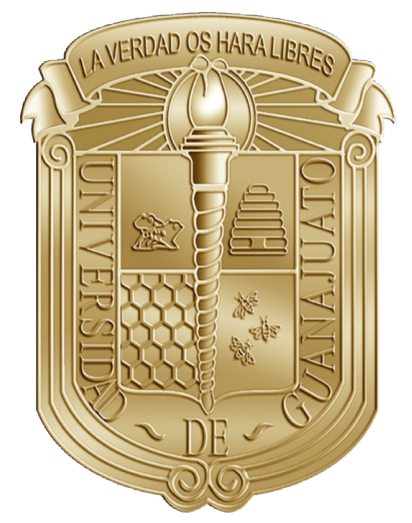


A la Universidad de Valladolid, y a la Escuela de Ingenierías Industriales, por permitirme realizar mis estudios de Doctorado. Les agradezco el haberme proporcionado el equipo y las instalaciones necesarias para terminar mis estudios y por hacerme sentir parte de esta gran institución como lo es la Universidad de Valladolid.

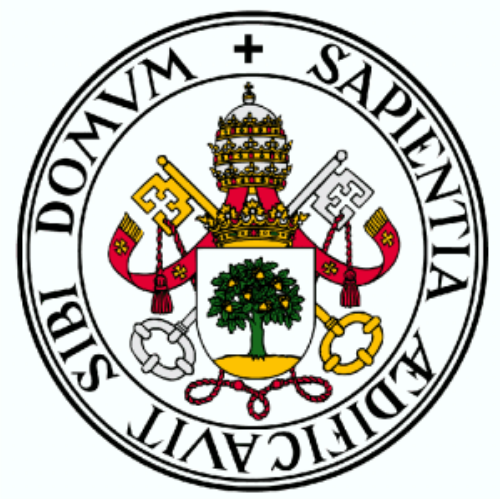


Al Consejo Nacional de Ciencia y Tecnología por la beca otorgada mediante el apoyo No. $348700 / 237491$.

- Título de trabajo: Diagnóstico de motores eléctricos para la localización de fallas incipientes.

- Autor: Paulo Antonio Delgado Arredondo.

- Asesor: Dr. René de Jesús Romero Troncoso.

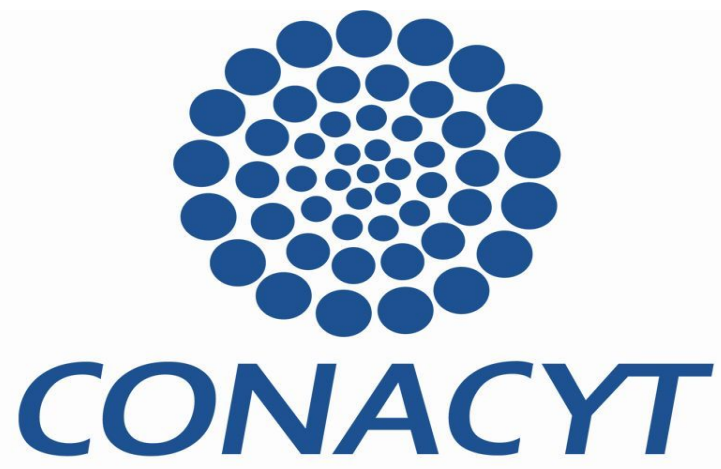




\section{Resumen}

Los motores de inducción son componentes críticos para la mayoría de las industrias y el monitoreo de su condición se ha convertido en una acción necesaria para detectar fallas; además el mantenimiento oportuno de los motores eléctricos es vital para mantener los complejos procesos de producción industrial. La monitorización de la condición de las máquinas eléctricas se puede realizar a través de la medición de distintas de sus señales. Al respecto, algunas de las más importantes son las vibraciones, las corrientes y el sonido. Para obtener el diagnóstico del motor es necesario analizar la señal capturada e identificar las frecuencias presentes en su espectro que están asociadas con fallos, el análisis puede llevarse a cabo durante la operación en estado estacionario o durante el transitorio de arranque del motor. Para señales capturadas en estado estable, el contenido frecuencial de las señales se obtiene generalmente con la transformada de Fourier, y para las señales capturadas en el transitorio de arranque, el contenido espectral y su evolución en el tiempo se obtienen por una descomposición tiempo-frecuencia de la señal.

En lo que respecta a la industria, las vibraciones son sin lugar a dudas las señales más utilizadas para diagnosticar, aunque prácticamente siempre a través de un análisis en régimen permanente. Por otra parte, existen muy pocas investigaciones relativas al uso de las vibraciones. Con respecto a la corriente, ocurre lo inverso: su presencia en la industria es mucho menor que las vibraciones, además de que las técnicas utilizadas son las más básicas, mientras que los centros de investigación han dedicado muchos esfuerzos en mejorar las técnicas basadas en el análisis de dicha señal, y en especial a través del transitorio, el cual ha demostrado ser fuente de mucha información. Finalmente, el sonido ha sido utilizado de for- 
ma muy precaria desde siempre por los técnicos de mantenimiento: les basta con escuchar un rodamiento, amplificando el sonido con un cilindro alargado colocado en el punto adecuado, para diagnosticar este componente. No obstante, su potencial al registrarlo adecuadamente y aplicar los aparatos matemáticos está todavía por descubrir, utilizándose de forma muy minoritaria en la industria, y habiéndose investigado poco o nada por parte de la comunidad científica.

Para enriquecer el conocimiento y complementar las abundantes investigaciones en torno al análisis de corrientes, merece la pena la exploración de metodologías de diagnóstico basadas en el análisis de vibraciones y sonido, debido a que cada señal amplifica mejor la presencia de un tipo de avería, y que, frente a un motor con un funcionamiento sospechoso, resulta conveniente combinar la información de cada una de ellas. El análisis de señales de sonido y el análisis de vibraciones, puede ofrecer grandes prestaciones para el diagnóstico de motores de inducción. En definitiva, el potencial del análisis de las vibraciones en régimen transitorio está todavía por descubrir. Teniendo en cuenta la importante información que se ha podido obtener del análisis de la corriente en transitorio, resulta evidente el interés de investigar qué se puede obtener con el transitorio de las vibraciones, que tan bien funcionan para diagnosticar motores en permanente en la industria. Por otra parte, el sonido, cuya información resulta relevante para los técnicos, incluso con un análisis precario, debe ser también motivo de estudio, aplicando lo conocido del análisis permanente en vibraciones y corrientes.

Una de las contribuciones de este trabajo es una nueva metodología de análisis para la detección de fallas en motores de inducción en estado estacionario. El diagnóstico de las fallas se hace por medio del análisis de señales de sonido y los resultados se corroboran con el análisis de señales de vibración que es una metodología ampliamente aceptada. Este enfoque propuesto utiliza la CEEMD para descomponer la señal en el dominio del tiempo en varias funciones de modo intrínseco (IMFs). Posteriormente, se calcula el marginal en frecuencia de la representación de Gabor; esto se calcula con el fin de obtener el contenido espectral de las IMFs en el dominio de la frecuencia e identificar los componentes espectrales asociados a las fallas. Esta nueva metodología propuesta arroja mejores resultados para la detectabi- 
lidad en comparación con otros trabajos publicados. Esta metodología propuesta se valida experimentalmente con la identificación de las fallas de dos barras del rotor rotas, desbalance mecánico y defectos en la pista externa del rodamiento.

Además de la metodología propuesta para el diagnóstico de motores en estado estable, en este trabajo también se presenta un estudio comparativo de diferentes técnicas de análisis tiempo-frecuencia que pueden utilizarse para detectar fallas en motores de inducción analizando señales de vibración durante el transitorio de arranque del motor. Las técnicas estudiadas son la distribución tiempo-frecuencia de Gabor (TFDG), el escalograma tiempo-frecuencia de Morlet (TFMS), la clasificación de señales múltiples (MUSIC) y la transformada de Fourier en tiempo corto (STFT). Las señales de vibración analizadas provienen de motores que presentan las siguientes fallas: una barra del rotor rota, dos barras rotas, desbalance y defectos en la pista externa del rodamiento. Los resultados obtenidos muestran la viabilidad de detectar fallas en motores de inducción utilizando el análisis espectral tiempo-frecuencia aplicado a señales de vibración. Esta metodología propuesta es aplicable cuando se pretende diagnosticar fallas mecánicas o para complementar un análisis de señales de corriente. Además, la metodología tiene aplicaciones en motores que se alimentan con inversores, en tales casos no se recomienda el análisis de señales de corriente debido a la mala calidad de la señal. 


\section{Abstract}

Induction motors are critical components for most industries and monitoring their condition has become a necessary action to detect faults; In addition, the timely maintenance of the electric motors is vital to maintain the complex processes of industrial production. The monitoring of the condition of electric machines can be carried out by measuring different signals. In this regard, some of the most important are vibrations, currents and sound. To obtain the motor diagnosis, it is necessary to analyze the captured signal and identify the frequencies present in its spectrum that are associated with faults, the analysis can be carried out during steady state operation or during the motor startup transient. For signals captured in steady state, the frequency content of the signals is generally obtained with the Fourier transform, and for the signals captured in the startup transient, the spectral content and its evolution in time are obtained by a time-frequency decomposition of the signal.

As far as the industry is concerned, vibrations are undoubtedly the most used signals to diagnose, although practically always through a steady state analysis. On the other hand, there is very little research concerning the use of vibrations. With regard to current, the reverse is true: its presence in the industry is much less than vibrations, and the techniques used are the most basic, while research centers have devoted much effort to improving the techniques based on analysis of this signal, and especially through the transient, which has proven to be a source of much information. Finally, the sound has been used in a very precarious way by the maintenance technicians: it is enough to listen to a bearing, amplifying the sound with an elongated cylinder placed at the appropriate point, to diagnose this component. However, its potential to properly register and apply the mathematical apparatus is 
still to be discovered, being used in a very minor way in the industry, and little or nothing has been investigated by the scientific community.

In order to enrich the knowledge and to complement the abundant research on current analysis, it is worth exploring diagnostic methodologies based on the analysis of vibrations and sound, because each signal amplifies better the presence of a type of fault, and that, in front of a motor with a suspicious operation, it is convenient to combine the information of each one of them. The analysis of sound signals and the analysis of vibrations, can offer great benefits for the diagnosis of induction motors. In short, the potential of transient vibration analysis is still to be discovered. Taking into account the important information that has been obtained from the analysis of the transient current, it is evident the interest to investigate what can be obtained with the transient vibrations, which work so well to diagnose permanent motors in the industry. On the other hand, sound, whose information is relevant to technicians, even with a precarious analysis, should also be a reason for study, applying the known of the permanent analysis in vibrations and currents.

One of the contributions of this work is a new analysis methodology for fault detection in induction motors at the steady state operation. The diagnosis of the faults is done by means of the analysis of sound signals and the results are corroborated with the analysis of vibration signals that is a methodology widely accepted. This proposed approach uses the CEEMD to decompose the time domain signal into several intrinsic mode functions (IMFs). Then, the frequency marginal of the Gabor representation is calculated, this is calculated to obtain the spectral content of the IMFs in the frequency domain and to identify the spectral components associated with the faults. This new proposed methodology yields better results for detectability compared to other published works. This proposed methodology is validated experimentally with the identification of the faults of two broken rotor bars, mechanical unbalance and defects in the outer race of the bearing.

In addition to the proposed methodology for the diagnosis of motors in the steady state operation, this paper also presents a comparative study of different techniques of time- 
frequency analysis that can be used to detect faults in induction motors by analyzing vibration signals during the startup transient of the motor. The techniques studied are the timefrequency distribution of Gabor (TFDG), the time-frequency Morlet scalogram (TFMS), Multiple Signal Classification (MUSIC) and the short time Fourier transform (STFT). The vibration signals analyzed come from motors with the following faults: a broken rotor bar, two broken rotor bars, unbalance and defects in the outer race of the bearing. The results show the feasibility of detecting faults in induction motors using the time-frequency spectral analysis applied to vibration signals. This proposed methodology is applicable when it is intended to diagnose mechanical faults or to complement an analysis of current signals. In addition, the methodology has applications in motors that are fed from an inverter, in such cases the analysis of current signals due to poor signal quality is not recommended. 


\section{Índice}

\begin{tabular}{|l|l|}
\hline Agradecimientos & I \\
\hline
\end{tabular}

\begin{tabular}{|l|l|}
\hline Agradecimientos institucionales & III
\end{tabular}

$\begin{array}{lll}\text { Resumen } & \text { VI }\end{array}$

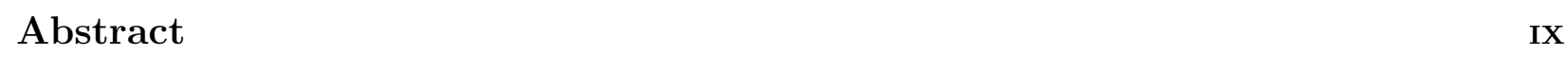

\begin{tabular}{lr}
\hline 1. Introducción & 1
\end{tabular}

1.1. Estado del arte . . . . . . . . . . . . . . . . . . . . . . . . 4

1.1.1. Tipos de alimentación . . . . . . . . . . . . . . 5 5

1.1.2. Magnitud física analizada . . . . . . . . . . . . . . 12

1.1.3. Técnicas de detección . . . . . . . . . . . . . . . . . . . . 19

1.1.4. Técnicas de diagnóstico . . . . . . . . . . . . . . . . . . . . . . . . . . 29

1.2. Planteamiento del problema . . . . . . . . . . . . . . . . . . . 31

1.2.1. Hipótesis . . . . . . . . . . . . . . . . . . . 31

1.2.2. Justificación . . . . . . . . . . . . . . . . . . . . . . 31

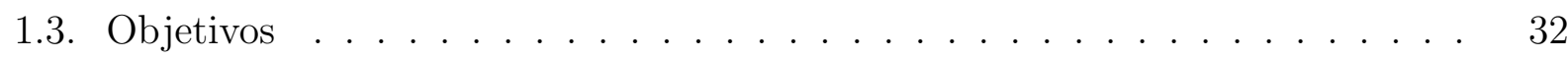

1.4. Organización de la tesis . . . . . . . . . . . . . . . . . . 33

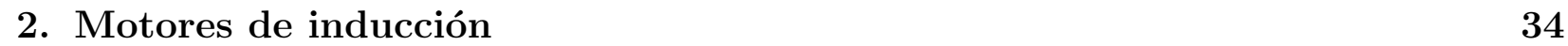

2.1. Partes principales . . . . . . . . . . . . . . . . . 35

2.2. Principio de funcionamiento . . . . . . . . . . . . . . . . . . . 39

2.3. Fallas en motores de inducción . . . . . . . . . . . . . . . . . . . . . . . 42 
2.3.1. Barras rotas . . . . . . . . . . . . . . . . . . . . . . 42

2.3.2. Rodamientos . . . . . . . . . . . . . . . . . . 43

2.3.3. Desbalance . . . . . . . . . . . . . . . . . . . . . 44

\begin{tabular}{lr}
\hline 3. Análisis espectral & 46
\end{tabular}

3.1. Señales no estacionarias . . . . . . . . . . . . . . . . . 46

3.2. Representación temporal y frecuencial. . . . . . . . . . . . . . . . . 47

3.3. Localización y el principio de Heisenberg-Gabor . . . . . . . . . . . . . 47

3.4. Frecuencia instantánea y retardo de grupo . . . . . . . . . . . . . . 48

3.5. Primera clase de solución: las descomposiciones atómicas . . . . . . . . . . 49

3.5.1. La transformada de Fourier en tiempo corto . . . . . . . . . . . . 50

3.5.2. Análisis tiempo-escala y la transformada wavelet. . . . . . . . . . . 51

3.5.3. De las descomposiciones atómicas a las distribuciones de energía . . . 52

3.6. Segunda clase de solución: distribuciones de energía . . . . . . . . . . . 55

3.6.1. Propiedades de las representaciones tiempo-frecuencia . . . . . . . . . 56

3.6.2. La clase de Cohen . . . . . . . . . . . . . . . . . . . 58

3.6.3. La clase afín . . . . . . . . . . . . . . . . . . . . 60

3.7. Distribución tiempo-frecuencia de Gabor . . . . . . . . . . . . . . . . . 61

3.8. Escalograma de Morlet . . . . . . . . . . . . . . . . . . . . . . . . 62

3.9. Extracción de información de una imagen tiempo-frecuencia . . . . . . . . 62

3.9.1. Momentos . . . . . . . . . . . . . . . . . . . 63

3.9.2. Marginales . . . . . . . . . . . . . . . . . . . . 64

3.10. Estimación de la densidad espectral de potencia . . . . . . . . . . . . . . 64

3.10.1. Descomposición armónica . . . . . . . . . . . . . . . 66

3.10.2. Multiple Signal Classification (MUSIC) . . . . . . . . . . . . 73

3.11. Métodos de descomposición empírica . . . . . . . . . . . . . . . . . 74

3.11.1. CEEMD . . . . . . . . . . . . . . . . . . . . . 78

\begin{tabular}{lr}
\hline 4. Experimentación & 80
\end{tabular}

4.1. Análisis en estado estable . . . . . . . . . . . . . . . . . . . . . . 80

4.1.1. Metodología . . . . . . . . . . . . . . . . . . . . . . 80 
4.1.2. Validación de la metodología propuesta . . . . . . . . . . . . . . . . . 82

4.1.3. Experimentación . . . . . . . . . . . . . . . . . . . . . 84

4.2. Análisis en el transitorio de arranque . . . . . . . . . . . . . . . . . . . . . 89

4.2.1. Metodología . . . . . . . . . . . . . . . . . . . . 89

4.2.2. Validación de la metodología propuesta . . . . . . . . . . . . . . . . . 89

4.2.3. Configuración experimental . . . . . . . . . . . . . . . . . . . . . . . 91

\begin{tabular}{|lr}
\hline 5. Resultados y discusión & 96
\end{tabular}

5.1. Resultados del análisis en estado estable . . . . . . . . . . . . . . . . . . . . 96

5.1.1. Caso de estudio: motor sano . . . . . . . . . . . . . . . . . . . . . . . 97

5.1.2. Caso de estudio: Dos barras del rotor rotas (2BRB) . . . . . . . . . . 99

5.1.3. Caso de estudio: Desbalance (UNB) . . . . . . . . . . . . . . . 102

5.1.4. Caso de estudio: Defectos en el rodamiento (BDF)] . . . . . . . . . . . 104

5.2. Resultados del análisis en el transitorio de arranque . . . . . . . . . . . . . . 118

5.2.1. Caso de estudio: motor sano . . . . . . . . . . . . . . . . . . . . . . . 119

5.2.2. Caso de estudio: Barras rotas . . . . . . . . . . . . . . . 120

5.2.3. Caso de estudio: Desbalance . . . . . . . . . . . . . . . . . 125

5.2.4. Caso de estudio: Defectos en el rodamiento . . . . . . . . . . . . . . . 126

\begin{tabular}{|rr}
\hline 6. Conclusiones y prospectivas & 129
\end{tabular}

6.1. Conclusiones del análisis en el estado estable . . . . . . . . . . . . . . . . . . 129

6.2. Conclusiones del análisis en el transitorio de arranque . . . . . . . . . . . . . 131

6.3. Prospectivas . . . . . . . . . . . . . . . . . 133

\begin{tabular}{ll}
\hline Contribuciones & 135
\end{tabular}

\begin{tabular}{lr}
\hline Referencias & 150
\end{tabular}

\begin{tabular}{ll}
\hline Glosario y siglas & 151
\end{tabular}

Glosario . . . . . . . . . . . . . . . . . . . . . . 151

Siglas . . . . . . . . . . . . . . . . . . . . 152 


\section{Índice de figuras}

2.1. Partes principales. . . . . . . . . . . . . . . . 36

2.2. Estátor. . . . . . . . . . . . . . . . . . . . 37

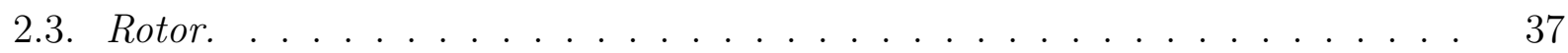

2.4. Jaula de ardilla. . . . . . . . . . . . . . . . . . . . . . . . 38

2.5. Jaula de ardilla. . . . . . . . . . . . . . . . . . . . . . . . . . . . . . . . . . . 39

2.6. Desarrollo del par inducido en un motor de inducción. . . . . . . . . . . . . 41

3.1. Representación simbólica de las ventanas tiempo-frecuencia para la a)STFT y la b)CWT. . . . . . . . . . . . . . . . . . . 52

4.1. Metodología propuesta para el estado estable. . . . . . . . . . . . . . . . . . . 81

4.2. Comparación entre la metodología propuesta y la FFT. a) Señal sintética. b) Espectro ideal. c) FFT de la señal. d) Marginal en frecuencia de la TFDG de las IMFs seleccionadas . . . . . . . . . . . . . . . . . . . . . . . . . . . . . . 83

4.3. Banco de pruebas usado para los ensayos en estado estable. a) Vista general. b) Vista frontal del motor donde se muestra la orientación de los ejes del acelerómetro. c) Vista lateral del motor donde se muestra la orientación de los ejes del acelerómetro. . . . . . . . . . . . . . . . . . . . . . . . . . . . . . 86

4.4. Rotor con 2 barras rotas. . . . . . . . . . . . . . . . . . . . 87

4.5. Polea con desbalance. . . . . . . . . . . . . . . . . . . 87

4.6. Rodamiento dañado. . . . . . . . . . . . . . . . . . . . 88

4.7. Metodología propuesta para el transitorio de arranque. . . . . . . . . . . . . . 90 
4.8. Validación de las técnicas propuestas. . . . . . . . . . . . . . . . . . . . . . . 94

4.9. Banco de pruebas usado para los ensayos durante el transitorio de arranque. $\quad 95$

4.10. Rotor con una barra rota. . . . . . . . . . . . . . . . . . . . . . . . 95

5.1. Primeras tres IMFs obtenidas de la señal de sonido para el motor sano. . . . 98

5.2. Espectro de las primeras tres IMFs obtenidas de la señal de sonido para el motor sano. . . . . . . . . . . . . . . . . . . . . . . . . 99 99

5.3. Primeras cuatro IMFs obtenidas de la señal de vibraciones para el motor sano. 100

5.4. Espectro de las primeras tres IMFs obtenidas de la señal de vibraciones para el motor sano. . . . . . . . . . . . . . . . . . . . . . . . . . . . . . 101

5.5. Primeras tres IMFs de la señal de sonido para el motor con dos barras rotas. 103

5.6. Espectro de la señal de sonido para a) el motor sano y b) el motor con dos barras rotas. . . . . . . . . . . . . . . . . . . . . . . . 107

5.7. Primeras cuatro IMFs de la señal de vibracines para el motor con dos barras rotas. . . . . . . . . . . . . . . . . . . . . . 108

5.8. Espectro de la señal de vibraciones para a) el motor sano y b) el motor con dos barras rotas. . . . . . . . . . . . . . . . . . . . . . . . . . 109

5.9. Primeras tres IMFs de la señal de sonido para el motor con desbalance. . . . 110

5.10. Espectro de la señal de sonido para a) el motor sano y b) el motor con desbalance. 111

5.11. Primeras cuatro IMFs de la señal de vibracines para el motor con desbalance. 112

5.12. Espectro de la señal de vibraciones para a) el motor sano y b) el motor con

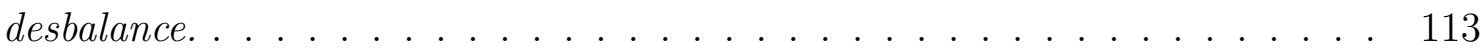

5.13. Primeras tres IMFs de la señal de sonido para el motor con defectos en el rodamiento. . . . . . . . . . . . . . . . . . . 114

5.14. Espectro de la señal de sonido para a) el motor sano y b) el motor con defectos en el rodamiento. . . . . . . . . . . . . . . . . . . . . . . . 115

5.15. Primeras cuatro IMFs de la señal de vibracines para el motor con defectos en el rodamiento. . . . . . . . . . . . . . . . . . . . . . 116

5.16. Espectro de la señal de vibraciones para a) el motor sano y b) el motor con defectos en el rodamiento. . . . . . . . . . . . . . . . . . . . . . . . . . 117 
5.17. Ventana Hamming de 512 puntos. . . . . . . . . . . . . . . . . . . . . . . 119

5.18. Ventana gaussiana de 512 puntos. . . . . . . . . . . . . . . . 120

5.19. Ventana gaussiana de 512 puntos. . . . . . . . . . . . . . . . . . . . 121

5.20. Descomposiciones tiempo-frecuencia para un motor sano. A) STFT. B) TFDG. C) TFMS. D) MUSIC. . . . . . . . . . . . . . . . . . . . . . . . . . . . 122

5.21. Descomposiciones tiempo-frecuencia para una barra del rotor rota. A) STFT. B) TFDG. C) TFMS. D) MUSIC. . . . . . . . . . . . . . . . . . . . . 123

5.22. Espectros obtenidos con las descomposiciones tiempo-frecuencia para dos barras del rotor rotas. A) STFT. B) TFDG. C) TFMS. D) MUSIC. . . . . . . 124

5.23. Espectros obtenidos con las descomposiciones tiempo-frecuencia para el caso de desbalance. A) STFT. B) TFDG. C) TFMS. D) MUSIC. . . . . . . . . . 125

5.24. Espectros obtenidos de la descomposición tiempo-frecuencia para el caso de defectos en el rodamiento. A) STFT. B) TFDG. C) TFMS. D) MUSIC. . . . 127 


\section{Índice de tablas}

5.1. Valores de frecuencias de falla detectadas en la señal de sonido para 2BRB. . 102 5.2. Valores de frecuencias de falla detectadas en la señal de vibraciones para 2BRB. 102 5.3. Valores de frecuencias de falla detectadas en la señal de sonido para el desbalance.104 5.4. Valores de frecuencias de falla detectadas en la señal de sonido para BDF. . 105 5.5. Detectabilidad en $d B$ para las fallas analizadas . . . . . . . . . . . . . 106

5.6. Detectabilidad en $d B$ para el estudio comparativo de técnicas para la identificación de fallas en un motor de inducción. . . . . . . . . . . . . . . . . . . . 128 


\section{Capítulo 1}

\section{Introducción}

Desde el inicio de la historia, el ser humano ha aprovechado su intelecto para hacer mejor y más fácil su vida. Esta mejora en sus condiciones de vida ha sido fundamentada con la creación de herramientas y máquinas, que le han permitido realizar tareas de una manera más sencilla y eficiente. Al mismo tiempo, la humanidad y estas máquinas han ido evolucionando gracias al desarrollo del conocimiento científico hasta convertirse en lo que son hoy en día. Las máquinas son los elementos en los cuales toda nuestra civilización actual basa su desarrollo y estilo de vida, en este hecho recae la importancia que estas tienen para el ser humano. El motor eléctrico es una de estas máquinas de gran importancia. Esta máquina ha contribuido en gran parte al desarrollo de la humanidad y en la actualidad es una de las maquinas más usadas en la industria, ya que más del $40 \%$ de la electricidad consumida mundialmente es usada para alimentar sistemas accionados por motores eléctricos [1]. Debido a su extendido uso, es necesario el monitoreo constante de su condición de operación, establecer intervenciones oportunas para su mantenimiento, la solución de problemas cuando estos se presentan y garantizar su correcto funcionamiento.

La evolución y enriquecimiento del conocimiento científico también ha generado ingenios matemáticos y metodologías para analizar elementos presentes en el mundo que nos rodea. Las metodologías para analizar señales han ido modificándose, desde analizar las señales en su representación más común, en el dominio temporal, como un registro de algún fenómeno 
físico en función del tiempo; hasta la utilización de operaciones matemáticas para su transformación a otro dominio. Una de estas operaciones matemáticas es la transformada de Fourier. Esta operación matemática nos permite conocer los componentes frecuenciales de una señal, todo esto con el fin de adquirir información que de otra manera no hubiese sido posible conocer. Asimismo, el estudio del contenido de frecuencias de una señal tiene vastas aplicaciones y está ligado a muchos campos de estudio, por mencionar algunos: los terremotos, el habla humana, las imágenes en medicina, los datos financieros, la música, además de un sin número de otros campos en los que puede ser aplicado el análisis espectral de señales.

El diagnóstico y monitoreo de fallas en los motores eléctricos no es la excepción de las áreas de estudio susceptibles al análisis espectral. Mediante el análisis espectral de señales de corriente, de vibraciones y de sonido es posible detectar fallas en tan importante máquina. Como se mencionó anteriormente, la representación temporal es comúnmente la primera y la más natural descripción de una señal, esto es debido a que todas las señales físicas son obtenidas en función del tiempo. Por otro lado, la representación en el dominio de la frecuencia obtenida por la transformada de Fourier, la cual se define en su forma general por la siguiente ecuación:

$$
X(f)=\int_{-\infty}^{+\infty} x(t) e^{-j 2 \pi f t} d t
$$

donde $x(t)$ es la representación temporal de la señal, $X(f)$ representa la Transformada de Fourier o Integral de Fourier de la señal $x(t), j$ es el valor imaginario $\sqrt{-1}$ y $f$ es la frecuencia [2]. Es una forma muy poderosa para describir una señal, esto porque el concepto de frecuencia esta compartido por muchos dominios en los cuales ocurren eventos periódicos.

El espectro obtenido a través de esta transformación, esencialmente muestra qué frecuencias están contenidas en la señal, así como sus amplitudes y fases, pero no en qué tiempo estas frecuencias ocurren. Es decir, si se analiza cuidadosamente el espectro $X(f)$, este puede ser visto como una función de coeficientes obtenidos por la expansión de la señal $x(t)$ en una familia de curvas infinitas, $e^{-j 2 \pi f t}$, para las cuales son completamente ilocalizables los 
intervalos de tiempo en los que estas aparecen. A pesar de esto, cuando se analizan señales estacionarias (señales en las cuales su contenido en frecuencia permanece constante) como las obtenidas en un motor de inducción durante su funcionamiento en estado estable, el resultado obtenido es el idóneo. En cambio, cuando se analizan señales no estacionarias con la transformada de Fourier, el resultado no será del todo útil, esto debido a que no está diseñada para conocer el intervalo de tiempo en el que las frecuencias variables de una señal no estacionaria ocurren. Esta problemática con señales no estacionarias es la que llevo al desarrollo de nuevas herramientas para el análisis espectral de señales.

Una de las contribuciones de este trabajo es una nueva metodología de análisis para la detección de fallas en motores de inducción en estado estacionario. El diagnóstico de las fallas se hace por medio del análisis de señales de sonido y los resultados se corroboran con el análisis de señales de vibración que es una metodología ampliamente aceptada. Este enfoque propuesto utiliza la CEEMD para descomponer la señal en el dominio del tiempo en varias funciones de modo intrínseco (IMFs). Posteriormente, se calcula el marginal en frecuencia de la representación de Gabor; esto se calcula con el fin de obtener el contenido espectral de las IMFs en el dominio de la frecuencia e identificar los componentes espectrales asociados a las fallas. Esta nueva metodología propuesta arroja mejores resultados para la detectabilidad en comparación con otros trabajos publicados. Esta metodología propuesta se valida experimentalmente con la identificación de las fallas de dos barras del rotor rotas, desbalance mecánico y defectos en la pista externa del rodamiento.

Además de la metodología propuesta para el diagnóstico de motores en estado estable, en este trabajo también se presenta un estudio comparativo de diferentes técnicas de análisis tiempo-frecuencia que pueden utilizarse para detectar fallas en motores de inducción analizando señales de vibración durante el transitorio de arranque del motor. Las técnicas estudiadas son la distribución tiempo-frecuencia de Gabor (TFDG), el escalograma tiempo-frecuencia de Morlet (TFMS), la clasificación de señales múltiples (MUSIC) y la transformada de Fourier en tiempo corto (STFT). Las señales de vibración analizadas provienen de motores que presentan las siguientes fallas: una barra del rotor rota, dos barras rotas, desbalance y defectos en la pista externa del rodamiento. Los resultados obtenidos muestran la viabilidad de detec- 
tar fallas en motores de inducción utilizando el análisis espectral tiempo-frecuencia aplicado a señales de vibración. Esta metodología propuesta es aplicable cuando se pretende diagnosticar fallas mecánicas o para complementar un análisis de señales de corriente. Además, la metodología tiene aplicaciones en motores que se alimentan con inversores, en tales casos no se recomienda el análisis de señales de corriente debido a la mala calidad de la señal.

\subsection{Estado del arte}

En la actualidad el desarrollo de investigaciones en cuanto al monitoreo y diagnóstico de motores de inducción se puede clasificar en cuatro grandes aspectos:

- Tipo de alimentación.

- Magnitud física analizada.

- Técnicas de detección.

- Técnicas de diagnóstico

El primer aspecto, como su nombre lo dice, se enfoca al tipo de alimentación del motor, es decir si es alimentado directamente con sinusoides provenientes de la red eléctrica o si es alimentado por un variador de frecuencia (inversor). Dependiendo del tipo de alimentación con la que cuente el motor bajo estudio, se decidirán las técnicas de detección y la magnitud física para el análisis más apropiadas.

El aspecto magnitud física analizada está enfocado al tipo de señal capturada, es decir el tipo de magnitud física medida, así como si esta magnitud es medida durante el transitorio de arranque del motor o si es medida durante el funcionamiento en estado estable. Las magnitudes físicas mas empleadas para el análisis son las corrientes y las vibraciones, pero no son las únicas, pues existen mas magnitudes físicas que pueden ser analizadas y que son aplicables dependiendo de la avería que se busca monitorizar y diagnosticar.

UG - UVa

Paulo Antonio Delgado Arredondo 
El tercer punto se refiere a las herramientas matemáticas que se han utilizado para poder analizar las señales provenientes de un motor de inducción. Así como sucede con los puntos anteriores, la elección de las distintas herramientas matemáticas para el análisis de señales dependerá de las características de las mismas señales, es decir, la elección de la técnica de análisis dependerá de si la señal a analizar es de vibraciones, corriente, sonido, etcétera, así como si la señal fue capturada durante el transitorio de arranque o durante el estado estable.

Por último, el cuarto aspecto se refiere a las técnicas que han sido utilizadas para poder obtener un diagnostico o la condición de operación del motor de manera automática. Estas técnicas van desde el uso de redes neuronales hasta el uso de lógica difusa. Todos estos aspectos acerca del estado del arte de años recientes serán analizados a fondo en las secciones siguientes.

\subsubsection{Tipos de alimentación}

La captura y análisis de señales de las distintas magnitudes físicas que están presentes en el funcionamiento de un motor de inducción, puede hacerse tanto de motores alimentados por la red eléctrica como de motores alimentados por variadores de frecuencia. Con el desarrollo de los dispositivos electrónicos de potencia que permitieron la creación de controladores de velocidad y debido a que actualmente el uso de variadores de frecuencia se ha extendido, y están presentes en prácticamente cualquier industria, la alimentación de motores conectados directamente de la red eléctrica está disminuyendo.

La historia del diagnóstico de fallas y la protección en motores eléctricos es tan antigua como los motores mismos [3]. Inicialmente se basaban únicamente en la protección de los motores contra sobre corrientes, sobre voltajes, derivaciones a tierra, etcétera. Sin embargo, con el aumento en la complejidad de las operaciones para las cuales los motores eran dispuestos y así mismo el aumento de las implicaciones económicas de los sistemas, el diagnóstico de las fallas también se hizo más complejo, para intentar ir de un mantenimiento correctivo, a preventivo y finalmente a predictivo, disminuir el número de paradas, y los cambios inne- 
cesarios; esto propició la mejora de las técnicas de diagnóstico. Las técnicas originalmente diseñadas para el diagnóstico de fallas en motores de inducción alimentados directamente de la red eléctrica pueden ser aplicadas a los motores alimentados por inversores, sin embargo se deben tomar en cuenta cuestiones como el ruido en la alimentación, un significativamente mayor contenido armónico, distorsiones de la señal, etcétera.

Las primeras investigaciones en cuanto a la detección de fallas en motores alimentados con inversores se remontan a la década de 1980 [4]. Una de estas es la investigación hecha por Thompson y Stewart [5], donde muestran que es posible detectar fallas tales como barras del rotor rotas y excentricidad analizando señales de corriente provenientes de motores alimentados por inversores.

A pesar del considerable tiempo durante el cual se han desarrollado investigaciones en cuanto al monitoreo y diagnóstico de motores de inducción alimentados por inversores, aun hoy en día es un tópico de gran interés debido a las implicaciones económicas que conlleva. En los últimos años se siguen desarrollando investigaciones para la detección de fallas en motores de inducción alimentados por inversores, esto debido a que las distintas condiciones de operación del motor como lo son variaciones en el nivel de carga a la que se somete el motor, cambios de velocidad, arranques y paradas continuas, las distintas topologías que se usan en los inversores, la gran gama de diseños que se usan en la fabricación de los motores, etcétera, son factores que dificultan la utilización de alguna metodología o análisis universal y esta razón es la causante del constante desarrollo de metodologías de análisis, monitoreo y diagnóstico.

Autores como Georgakopoulos et al. [6] estudian la capacidad de detectar componentes frecuenciales específicos asociados a barras rotas en motores alimentados con inversor. En su trabajo aseguran una identificación sencilla de los componentes. Una de las aportaciones de este trabajo es que detectan la falla de barras rotas en motores con baja carga y cuando la falla esta en estados incipientes, además hacen la identificación de la falla tanto durante el funcionamiento en estacionario del motor como en el transitorio de arranque. A pesar de los avances, la detección de la falla en el caso del transitorio es meramente cualitativa a través de la comparación de amplitudes de las distintas descomposiciones que obtienen con 
la transformada wavelet discreta.

En el trabajo de Sung-Kuk y Jul-Ki [7] se presenta una aproximación no paramétrica para la detección de barras rotas en motores alimentados con inversor. Para realizar el diagnóstico de las barras rotas, en este trabajo se propone inyectar voltajes de alta frecuencia (20V a 150 $\mathrm{Hz}$ y $500 \mathrm{~Hz}$ ) en un motor con una frecuencia de rotación del rotor de $10 \mathrm{~Hz}$, los cuales están sobrepuestos a la frecuencia fundamental de alimentación, esto durante el estacionario del motor. La inyección de la señal de alta frecuencia se hace con el propósito de crear cambios en la resistencia de las barras del rotor lo cual se manifestará en el espectro de la señal de corriente del estátor. La metodología mantiene su efectividad aun en distintas condiciones de carga. A pesar de lo novedoso de la metodología, posee el gran inconveniente de la inyección de voltajes lo cual hace que el método sea difícil de implementar, además de que solo es aplicable para detectar barras rotas en el rotor.

En la investigación realizada por Chua et al. [8] se proporciona una técnica para detectar las fallas de barras rotas y fallas en los rodamientos utilizando una combinación de técnicas. Por un lado utilizan ICA (acrónimo de Independent Component Analysis) para extraer características en el dominio del tiempo y la FFT para las características en el dominio de la frecuencia. Además, para afrontar el problema del ruido introducido por los inversores hacen una reducción del ruido en la señal. Una vez que extraen las características en el dominio del tiempo y en el dominio de la frecuencia conjuntan esta información para usarla en el clasificador. La metodología propuesta presenta una eficiencia perfecta en la clasificación y detección de las fallas. Este método es novedoso en cuanto a la utilización del análisis conjunto en tiempo y en frecuencia, además de que presentan una eficiencia perfecta en el diagnóstico, sin embargo, las características que obtienen en frecuencia son las amplitudes de los componentes espectrales asociados a las barras rotas que se encuentran alrededor de la frecuencia fundamental, estas amplitudes y su localización, dependerán del deslizamiento, del nivel de carga del motor y además de la severidad de la falla. Este trabajo resulta interesante como posible aplicación de este método a otro tipo de fallas y a fallas con distintos grados de severidad.

En el trabajo realizado por Pons-Llinares et al. [9] se propone una metodología para el diagnóstico de excentricidad combinada en motores de inducción alimentados por inversor 
analizando la señal de corriente en el transitorio usando la AWT (acrónimo de Analitic Wavelet Transform). A partir de esta metodología se obtiene una representación gráfica con gran detalle de los componentes frecuenciales de la señal. A pesar de que la AWT permite mostrar la evolución de las componentes con suficiente nitidez, su uso se desaconseja, pues la forma en la que las wavelets cambian a lo largo de la frecuencia gracias al escalado de dicha transformada, genera una resolución a lo largo del plano que es adecuada solamente para evoluciones parabólicas, lo cual no es el caso de las componentes de máquinas eléctricas.?

Duque-Perez et al. [10] hacen pruebas de laboratorio a motores alimentados con inversor con excentricidad creada artificialmente para identificar tantas firmas de falla como sea posible. Muestran que las componentes espectrales alrededor de los armónicos de la fuente de alimentación contienen la información acerca de la excentricidad. El análisis de la falla de excentricidad lo hacen en señales de corriente del estator, además de que el propósito de analizar un motor alimentado por inversor es para obtener un criterio de diagnóstico que sea independiente del tipo de alimentación. Sin embargo, el estudio que se hace esta inconcluso, teniendo que considerar fuentes de alimentación con una menor relación señal/ruido en trabajos prospectivos.

Georgakopoulos et al. [11] presentan una metodología para detectar las fallas de barras rotas y excentricidad mixta en motores alimentados con inversor, para esto utilizan la FFT y la DWT (acrónimo de Discrete Wavelet Transform). Analizan en estado estable y en el transitorio de arranque. La metodología que proponen analiza las señales de corriente del estátor (salida del inversor) y las señales de corriente de entrada del inversor. Una de las ventajas de la metodología es la capacidad de detectar las fallas analizadas en estados incipientes de las fallas, con el motor en distintas condiciones de carga y a distintas frecuencias de alimentación. A pesar de que las fallas se detectan con la metodología propuesta, la detección se hace por comparación de las señales del motor defectuoso con las señales del motor sano y no se proporcionan umbrales para la determinación de la presencia de las fallas.

Ece y Basaran [12] proponen una metodología para detectar diferentes tipos de fallas en motores alimentados con inversor usando la transformada Wavelet y clasificadores. Esta metodología es probada en seis motores distintos, uno de ellos es un motor sano y los otros cinco presentan fallas. Dos motores tienen fallas en los rodamientos, un motor tiene tres barras 
rotas, un motor tiene cinco barras rotas y otro un cortocircuito en el devanado del estator. Una de las ventajas de la metodología propuesta en este trabajo es que no es necesario conocer nada acerca del inversor y tampoco la velocidad del motor, el cual es uno de los inconvenientes en muchas otras metodologías. A pesar de esta ventaja, la determinación de la presencia de una falla se hace por comparación con el motor sano, y en este trabajo al no buscar componentes asocidos a tipos específicos de fallas hace inconveniente su aplicación a un caso aleatorio, debido a que solo se sabrá que hay una falla en el motor, pero sin saber a ciencia cierta cual es la falla.

Yahia et al. [13] presentan un estudio experimental de un motor con el rotor dañado y alimentado con un inversor. El objetivo principal de este trabajo es evaluar la viabilidad del análisis de corrientes de un motor alimentado por inversor para detectar la falla de barras rotas en el rotor, se analizan motores con una barra del rotor rota y dos barras rotas. Además, se analiza la efectividad del a técnica de análisis de corrientes a distintas frecuencias de alimentación. Una de las aportaciones del análisis experimental realizado aquí es que muestran que a bajas velocidades el diagnostico puede dificultarse debido al traslape de los componentes de falla y la frecuencia fundamental de la alimentación, además el análisis es complicado debido a la presencia de ruido en la alimentación y también demuestran que, en los inversores frecuencias de conmutación menores a $12 \mathrm{KHz}$ aumentan la dificultad del diagnóstico.

Fernandez-Temprano et al. [14] presentan un método para clasificar fallas basado en un análisis discriminante lineal aplicado a la detección de la falla de barras rotas en motores de inducción alimentados con inversor. Hacen la detección de la falla a diferentes cargas del motor y alimentado a diferentes frecuencias. Las fallas son progresivas desde un motor sano hasta uno con una barra totalmente rota. Con la metodología propuesta pueden determinar el grado de afectación en las barras del motor, además de identificar la falla en distintas condiciones de carga del motor. Una de las aportaciones interesantes en este trabajo es el análisis progresivo de la falla, lo cual es de gran importancia para lograr un mantenimiento predictivo.

El trabajo realizado por Mitra y Koley [15] propone una metodología para el análisis de señales de vibración en motores alimentados por VFD (acrónimo de Variable Frequency 
Drive), detectan fallas en los rodamientos y además contemplan la influencia de vibraciones externas. Esta detección la hacen con la ayuda de la FFT. La detección de las frecuencias características asociadas a la falla es difícil con FFT y se plantean el uso de detección inteligente a futuro. La más importante aportación de este trabajo es el análisis de los efectos de vibraciones externas en las vibraciones del motor. Una de las desventajas del análisis empleado en este trabajo es la poca tolerancia al ruido de fondo de la FFT. En este sentido hay una gran oportunidad de investigar tanto nuevas metodologías para el análisis de señales de vibraciones a si como los efectos de vibraciones externas en la captura y el análisis de vibraciones de un motor de inducción.

En su investigación, Wang et al. [16] hacen la detección de fallas utilizando la FFT combinada con ICA. Detectan barras rotas y defectos en los rodamientos en motores alimentados con inversor a diferentes frecuencias. El diagnóstico se puede hacer sin el deslizamiento del motor, ya que se estiman los valores de las frecuencias de fallo con los parámetros del motor. El algoritmo empleando es robusto, flexible y fácil de implementar. Sin embargo, el método utiliza la FFT para obtener el espectro de la señal de corriente y de ahí toma las amplitudes de las frecuencias de falla estimadas, esto es una desventaja del método, debido a la baja tolerancia de la FFT al ruido. Además, bajo distintas condiciones de carga del motor, el deslizamiento puede variar significativamente haciendo muy complicado estimar las frecuencias de fallo de manera precisa utilizando solamente los parámetros de la placa de características del motor, situación que provocaría que el método fracase.

En el trabajo de Romero-Troncoso et al. [17] hacen una comparación y evaluación de metodologías para detectar la falla de barras rotas en tres condiciones distintas, desde un desarrollo temprano de la falla hasta el momento en que la barra está completamente rota. El análisis se hace en motores de inducción alimentados con inversor a tres frecuencias distintas y alimentados por la línea; además se examina a tres diferentes condiciones de carga. Las metodologías que se analizan son la FFT y MUSIC (acrónimo de Multiple Signal Classification), las cuales se evalúan aplicadas directamente a las señales. También se evalúa la FFT en combinación con la WT (acrónimo de Wavelet Transform) y con la EMD (acrónimo de Empirical Mode Decomposition). Además, también se evalúa el algoritmo MUSIC combinado con la EMD. Las técnicas que mejor se desempeñan son MUSIC y MUSIC en combinación 
con la EMD, ya que son capaces de detectar las fallas en la mayoría de las condiciones de falla y carga. Este resultado es debido a que MUSIC, desde un punto de vista teórico, permite separar la señal en dos subespacios, uno relacionado a la señal de interés y el otro al ruido, esto permite que se tengan espectros con poco o nada de ruido, por esta razón el uso de este algoritmo supera a la FFT en el análisis de señales de corriente de motores alimentados por inversor.

En el trabajo de Pons-Llinares et al. [18] proponen el uso de la AST (acrónimo de Adaptive Slope Transform) para analizar el transitorio de arranque de motores de inducción alimentados con inversor y poder diagnosticar la excentricidad mixta, esto con variaciones de carga, frecuencia, con y sin compensación de deslizamiento. Localizan y grafican la evolución de los armónicos asociados a la excentricidad en motores alimentados por inversor. Una de las aportaciones interesantes de este trabajo es que muestran la evolución de los componentes asociados a la excentricidad mixta en el transitorio de arranque del motor, en base a los resultados que se presentan esta técnica muestra ser viable para su aplicación en el diagnóstico de otro tipo de averías.

En recientes publicaciones, como la realizada por Romero-Troncoso et al. [19] se presenta una metodología de análisis de señales de corriente de un motor alimentado por inversor durante el transitorio de arranque y durante el estado estable. La metodología propuesta es una combinación de la CEEMD (acrónimo de Complete Ensemble Empirical Mode Decomposition) y MUSIC. Como se mencionó anteriormente, el algoritmo MUSIC posee la característica de que sus resultados no se ven afectados, al menos no tan significativamente como otras técnicas de análisis espectral, cuando la señal posee una baja relación señal/ruido, sin embargo cuando se busca tener amplitudes en los espectros que estén relacionadas directamente con las amplitudes en la señal en tiempo, no se aconseja el uso de MUSIC ya que suele decirse que el espectro que se obtiene por medio de esta técnica es un pseudoespectro. Una de las razones de nombrarlo pseudoespectro, es debido a que no refleja fielmente los verdaderos valores de amplitud de los componentes de la señal. Además de que debe tenerse un conocimiento previo de las características del espectro de la señal, esto a consecuencia de que el algoritmo MUSIC puede generar frecuencias espurias, es decir, componentes frecuenciales que realmente no están en la señal. En cuanto a la CEEMD muestra su capacidad de 
eliminar componentes no deseados de la señal, lo cual es realmente útil en señales con una baja relación señal/ruido.

Por último, en el trabajo realizado por Yang et al. [20] se realiza un estudio de la señal de corriente de un motor de inducción alimentado con inversor con el fin de generar conocimiento y mejorar el diagnóstico de la presencia de fallas en motores tales como barras rotas y fallas en rodamientos.

\subsubsection{Magnitud física analizada}

En los centros de investigación la metodología de estudio más popular y a la que se enfocan gran cantidad de investigaciones para la detección de fallas y diagnóstico en motores de inducción es el análisis de señales de corriente del estátor o MCSA (acrónimo de Motor Current Signature Analysis) [3,21,25]. La metodología MCSA utiliza un sensor no invasivo para obtener las señales de corriente del estátor y un sistema de adquisición de datos para adquirir la señal [26]. Este sensor podría ser de efecto Hall. Posteriormente esta señal de corriente se analiza para determinar su contenido espectral y buscar las características de los componentes asociados a diferentes fallas 27,29$]$. Las metodologías que usan MCSA permiten el diagnóstico de fallas en motores de una forma relativamente no invasiva [30], siempre y cuando la localización de los cables de alimentación del motor sea de fácil acceso. Esta metodología tiene la ventaja de que es fácil de implementar además de que proporciona buenos resultados fundamentalmente en el diagnóstico de fallas en el rotor [30]. Sin embargo, bajo ciertas condiciones su aplicación no es del todo idónea, lo cual es evidente en motores alimentados por inversor debido a que las señales de corriente capturadas tienen una baja relación señal/ruido, lo que dificulta la identificación de las fallas.

En lo que respecta a la industria, las vibraciones son sin lugar a dudas las señales más utilizadas para diagnosticar, aunque prácticamente siempre a través de un análisis en régimen permanente. Por otra parte, existen muy pocas investigaciones relativas al uso de las vibraciones. En esta metodología se aplican técnicas de análisis a señales de vibraciones para realizar el diagnóstico de fallas en motores de inducción. Las señales de vibración se 
pueden obtener de tres ejes espaciales, es decir, x, y y z [31]. Esta metodología de estudio es particularmente adecuada para la determinación de fallas mecánicas. La medición de las vibraciones se realiza utilizando acelerómetros como sensores primarios. Las señales de vibración se registran entonces con un sistema de adquisición de datos [26]. Las vibraciones en un motor de inducción se ven afectadas por pares vinculados a averías mecánicas, y en el caso de fallas eléctricas, por cambios en el campo magnético que provocan aparición de pares.

La metodología de análisis de señales de vibración es una técnica no invasiva, ya que no es necesario intervenir de ninguna manera en la operación del motor y además es una metodología eficiente para encontrar fenómenos asociados a las fallas que se pudieran presentar en un motor de inducción [32]. Además, el análisis de vibraciones tiene la ventaja de que sus resultados son menos dependientes del tipo de alimentación del motor que la metodología MCSA, y de que los resultados de su aplicación pueden ser equiparables con los que se pueden obtener con MCSA al diagnosticar barras rotas o incluso mejores si se diagnostican fallas mecánicas [33. A pesar de lo anterior, esta metodología tiene algunas desventajas, una de ellas es que su implementación requiere el uso de acelerómetros como sensores básicos que deben colocarse cerca o sobre el motor, aspecto que no siempre es posible lograr. Otra de las desventajas de la metodología de análisis de señales de vibración es que tienen una baja relación señal/ruido, lo que en ocasiones dificulta la detección de componentes espectrales de baja amplitud asociados a fallas. Se han realizado algunos trabajos para la detección de fallas en motores de inducción analizando señales de vibración. En estos trabajos, las señales capturadas del motor se llevan al dominio de la frecuencia y al dominio tiempo-frecuencia, dependiendo si son capturadas en el estado estable o en el transitorio de arranque. En [34,35], el diagnóstico del motor se hace analizando las señales de vibración durante el transitorio de arranque y en otros trabajos, como en [36.37] se analizan señales capturadas en estado estable.

En el trabajo realizado por Climente-Alarcon et al. [34] se evalúa la aplicación de una metodología de diagnóstico para la falla de barras rotas que analiza señales de vibración en el transitorio de arranque. Esta metodología de diagnóstico utiliza la distribución tiempofrecuencia de Zhao-Atlas-Marks. El diagnóstico de la falla en el transitorio de arranque puede 
servir para validar un diagnostico incierto en señales de corriente. Los autores seleccionan esta distribución tiempo-frecuencia debido a la reducción de los términos cruzados. La principal aportación de este trabajo es la demostración de que es posible la detección de barras rotas en el rotor mediante el análisis de señales de vibración. Este trabajo también muestra que una avería que típicamente se diagnostica con un análisis de señales de corriente se puede diagnosticar con señales de vibración. Una de las desventajas de esta metodología es el gran contenido armónico y de ruido en la señal y por ende en los espectros mostrados, situación que no permite visualizar claramente las frecuencias de interés a menos que se trate de un experto el que visualice dichos espectros. Asimismo una mejora a lo realizado aquí es extender su aplicación a otro tipo de fallas.

En el trabajo realizado por Pate y Giri [35], se desarrolla un método de diagnóstico que combina la EMD y la DWT para determinar el daño presente en el rodamiento de un motor de inducción. Como punto de partida los autores establecen que un motor en condiciones normales de operación tiene vibraciones con ciertas características, las cuales se modifican cuando se presenta una falla. La EMD es empleada en esta metodología para separar los componentes de la señal en distintos modos de oscilación, es decir descompone la señal en funciones que poseen desde las oscilaciones más rápidas a las más lentas. Una vez obtenidas estas componentes de oscilación que se llaman funciones de modo intrínseco, se obtiene la densidad espectral de potencia de las primeras cuatro funciones de modo intrínseco. A su vez la DWT se aplica a la función de modo intrínseco que posea la mayor amplitud y se obtiene la densidad espectral de potencia a las descomposiciones obtenidas con la DWT. Cuando ya se cuenta con las densidades espectrales de potencia mencionadas anteriormente, se buscan componentes en frecuencia relacionados con la falla de rodamientos. La metodología propuesta tiene el inconveniente del mezclado de modos de la EMD además de que la metodología solo se aplica a fallas en rodamientos, y sería interesante explorar la detección de otro tipo de fallas en señales de vibraciones.

El análisis del sonido es otra metodología de estudio que se ha utilizado para el diagnóstico de fallas en motores de inducción. Sin embargo, esta técnica no ha sido tan ampliamente empleada como la metodología MCSA y el análisis de vibraciones. Esta metodología es simple 
de implementar, es no invasiva y el análisis se realiza a frecuencias en el rango audible para humanos, es decir de $20 \mathrm{~Hz}$ a $20 \mathrm{kHz}$. La instrumentación consiste en un micrófono convencional situado lo más cerca posible del motor en análisis y un sistema de adquisición de datos. Con esta técnica, es posible obtener un diagnóstico tal como se obtendría con el análisis de vibraciones. Sin embargo, su desventaja es su sensibilidad al ruido acústico externo, que debe evitarse siempre que sea posible [37], por ejemplo, empleando micrófonos direccionales.

En cuanto a trabajos realizados usando el análisis de señales de sonido, Salazar-Villanueva e Ibarra-Manzano [38], donde proponen un enfoque basado en la EMD (acrónimo de Empirical Mode Decomposition) y la FFT para analizar señales de sonido con el fin de detectar desbalances mecánicos y defectos en los rodamientos de motores de inducción. La metodología que los autores proponen puede determinar la presencia de defectos en rodamientos y desbalance mecánico, sin embargo, al obtener el espectro de las señales de sonido estas contienen gran cantidad de ruido de fondo, haciendo que los resultados no sean tan claros. Una de las mejoras a esta metodología es utilizar una técnica de descomposición empírica mejorada que separe la señal de mejor manera sin que haya mezclado de modos en las descomposiciones y una herramienta de análisis espectral que tenga más tolerancia al ruido que la FFT. Además de plantear el diseño de un sistema de adquisición de datos que capture señales aisladas de ruidos externos y con una buena relación señal/ruido.

Ackay y Germen [39] proponen una metodología para identificar barras del rotor rotas y defectos en rodamientos en motores de inducción probados bajo diferentes condiciones de carga. Las señales sonoras se adquieren con cinco micrófonos colocados hemisféricamente alrededor del motor. Además utiliza un algoritmo híbrido para calcular la PSD (acrónimo de Power Spectral Density) y realizan el diagnóstico observando cambios de densidad para determinar la presencia de fallas. Una de las aportaciones interesantes de este trabajo es la captura de las señales de sonido de los motores de inducción, el sonido es capturado a una frecuencia de $44.1 \mathrm{KHz}$, esta es la frecuencia de muestreo estándar para discos compactos de audio digital. Además, la captura de las señales de sonido se realiza mediante cinco micrófonos colocados alrededor del motor en cuestión. Esta aportación es muy interesante ya que la propagación del sonido no será uniforme en todas las direcciones posibles, y al obtener señales 
de múltiples micrófonos colocados en distintas posiciones se tiene una mejor caracterización del sonido emitido por el motor. Además, en el algoritmo que utilizan, la información de los cinco micrófonos se utiliza en conjunto para obtener la PSD de las señales y conseguir el diagnóstico del motor. Una de las desventajas en este trabajo es que no se buscan frecuencias especificas relacionadas a las fallas, esto puede ser un inconveniente ya que en un entorno real no se sebe ciertamente la condición del motor, y a lo sumo esta metodología dirá si hay una falla o no, pero no cuál es la falla.

Glowacz [40] propone un método no invasivo de detección temprana de fallas para barras del rotor rotas y bobinas del rotor en cortocircuito. El análisis se realiza utilizando coeficientes de relación de área de registro y MUSIC, y la clasificación se hace con el clasificador de vecino más cercano y el clasificador de Bayes. El diagnostico se hace analizando rangos de frecuencia en el espectro obtenido con MUSIC y utilizando los clasificadores, es decir no se buscan frecuencias especificas asociadas a fallas. En este sentido el método propuesto parece no ser de uso general para analizar motores en los cuales se desconoce por completo la falla que presentan, ya que solo se analizan características o cambios en el espectro entre un motor sano y uno con falla.

Germen et al. [41] utilizan un método para la detección de fallas basado en la correlación y la DWT para extraer las características de la señal, que se utilizan para la clasificación de fallas con el método de mapas auto-organizados. Las fallas que se analizan en este trabajo son: tres barras del rotor rotas, desalineamiento en el rodamiento, defecto en una bola del rodamiento y cortocircuito en el devanado del estator. La captura de las señales de sonido se hace con cinco micrófonos y de manera hemisférica debido a la no uniformidad de propagación del sonido alrededor del motor. Una de las aportaciones más significativas del trabajo, además de la metodología de diagnóstico propuesta es que encuentran que distintas condiciones de falla emiten sonidos con distintas características. En este trabajo más allá de buscar componentes espectrales asociados a fallas como se hace comúnmente en las metodologías basadas en MCSA o en el análisis de vibraciones, analizan y detectan patrones de sonido característicos a cada una de las fallas.

García-Pérez et al. [37] proponen una metodología que utiliza el algoritmo MUSIC en señales de sonido y vibración para la detección de fallas en motores de inducción, incluyen- 
do el desbalance mecánico, barras del rotor rotas y defectos en rodamientos. La principal aportación de este trabajo es la utilización del sonido como medio de diagnóstico, además se analizan señales de vibración que corroboran los resultados. Sin embargo y a pesar de la utilidad de la metodología propuesta, la magnitud de los componentes espectrales detectados no se puede asegurar que sea la correcta debido a las características del algoritmo MUSIC. Este algoritmo genera un pseudoespectro en el cual las magnitudes de los componentes no necesariamente corresponden a los de la señal analizada y además pueden presentarse frecuencias espurias si no se escogen de manera correcta los eigenvalores correspondientes al subespacio de la señal y al subespacio de ruido.

Otra metodología que ha sido implementada para el diagnóstico de fallas, es el análisis de emisiones acústicas, que mide las señales acústicas en la gama de ultrasonidos, es decir a frecuencias mayores a las audibles por el ser humano. Esta metodología de análisis generalmente se aplica al diagnóstico de fallas mecánicas en motores de inducción. La captura de emisiones acústicas proporciona una alta relación señal/ruido en los datos obtenidos. El análisis de señales de emisión acústica ofrece ventajas sobre el análisis de vibraciones, lo que permite una detección más temprana y una mejor identificación de fallas [42].

Algunos autores como Choudhury y Tandon [43] demuestran la utilidad de la técnica de emisión acústica para detectar defectos en los rodamientos. Las frecuencias de muestreo para el análisis de señales de emisión acústica son superiores a $100 \mathrm{KHz}$, esto significa que es necesario metodologías de diagnóstico que no sean demandantes computacionalmente. En este trabajo se utiliza un método que contabiliza eventos de gran amplitud en la señal. Estos eventos en la señal de emisión acústica sirven para determinar si hay o no defectos en alguno de los componentes del rodamiento, conjuntamente sirven para determinar el tamaño de los defectos y su distribución.

He et al. 44 presentan un estudio experimental para investigar las características de la emisión acústica en rodamientos con defectos y validan la relación entre la señal de emisión acústica y las condiciones operativas del rodamiento. En ese trabajo se demuestra que la emisión acústica es más efectiva que el análisis de señales de vibración para diagnosticar fallas en rodamientos en estados incipientes de la falla. En este trabajo también demuestran 
que la velocidad de rotación del rodamiento tiene una fuerte influencia en la emisión acústica del motor. En base a los resultados que muestran, parece interesante la posibilidad de explorar la utilización de la emisión acústica en el diagnóstico de otro tipo de fallas mecánicas.

El trabajo de Al-Dossary et al. 45] proporciona la correlación entre el tamaño del defecto en los rodamientos con parámetros específicos de emisión acústica. El principal objetivo de este trabajo es el de caracterizar el tamaño de defectos en rodamientos mediante la aplicación de la emisión acústica. La principal aportación de este estudio es que se puede establecer una relación entre el tamaño de los defectos y ciertas características de las señales capturadas.

Otros trabajos como el de Halme y Andersson [46] presentan una revisión del estado de la técnica para el diagnóstico de rodamientos utilizando vibraciones y señales de emisión acústica. En este trabajo se muestran la gran variedad de condiciones de operación de rodamientos que se pueden determinar analizando señales de emisión acústica. Se analizan aplicaciones para determinar defectos en las pistas y en las bolas, fatiga, lubricación inadecuada, contaminación en lubricantes, desgaste, etcétera.

Elforjani y Mba [47,48] presentan los resultados que evalúan el potencial de la tecnología de emisión acústica para detectar y localizar defectos naturales en rodamientos y ejes. Un aspecto interesante de este par de trabajos es la monitorizacon y caracterización de la evolución de los defectos en rodamientos y en ejes. Este estudio muestra que es posible realizar un seguimiento de la evolución de fallas, este hecho es importante, sobre todo por la posible aplicación a otro tipo de fallas, ya que uno de los retos del diagnóstico es determinar condiciones de falla en etapas iniciales.

Caesarendra et al. [49] presentan una revisión del uso de la técnica de emisión acústica en el monitoreo de motores y la detección de fallos tempranos. El fallo que se analiza es en rodamientos, el análisis se realiza en tres casos de estudio relacionados a tres distintas velocidades de operación, además proponen un método para extraer características de señales de emisión acústica que permitan un diagnostico en etapas incipientes del diagnóstico.

A pesar de las ventajas del análisis de emisiones acústicas sobre el análisis de vibraciones y del análisis de sonido, requiere una implementación más compleja [50], mayores frecuencias de muestreo y tiempos de procesamiento considerablemente más largos, las cuáles son sus 
principales desventajas.

\subsubsection{Técnicas de detección}

En la literatura científica existen una gran variedad de herramientas matemáticas que se han utilizado para poder analizar las señales provenientes de un motor de inducción. Así como sucede con las magnitudes físicas, la elección de las distintas herramientas matemáticas para el análisis de señales dependerá de las fallas que se pretende diagnosticar y de las mismas señales, es decir, la elección de la técnica de detección dependerá de si la señal que se pretende analizar es de vibraciones, corriente, sonido, etc., y de si la señal fue capturada durante el transitorio de arranque o durante el estado estable.

El conjunto de técnicas de detección para el diagnóstico de fallas de motores de inducción mediante el análisis de las señales de vibración durante el transitorio de arranque, no es tan amplio en comparación con el conjunto de técnicas que se han usado para el análisis de señales de corriente. El análisis de señales de vibración y la metodología MCSA, se enfocan en la localización de componentes espectrales asociados a las fallas para hacer un diagnóstico de las condiciones de operación del motor.

La técnica de detección o herramienta matemática que generalmente se utiliza es la transformada de Fourier, que traduce una señal del dominio de tiempo al dominio de la frecuencia. La aplicación de la transformada de Fourier proporciona todo el contenido de frecuencias de una señal, sin embargo, esta técnica solo es conveniente para su aplicación a señales estacionarias e inherentemente no permite observar la evolución del contenido de frecuencias de la señal en el tiempo. Esta es la razón por la cual se han desarrollado herramientas de análisis para extender las capacidades de la transformada de Fourier, tales como las descomposiciones lineales tiempo-frecuencia (transformada de Fourier en tiempo corto y la transformada wavelet) y las descomposiciones cuadráticas tiempo-frecuencia.

Estas herramientas que se extienden a partir del análisis de Fourier, permiten conocer

UG - UVa

Paulo Antonio Delgado Arredondo 
simultáneamente el contenido en frecuencia que posee una señal y su evolución en el tiempo. Esto es muy útil para señales no estacionarias, como las obtenidas del transitorio de arranque de un motor de inducción, debido a que el contenido espectral característico del funcionamiento normal del motor y las firmas asociadas con fallas evolucionan con el tiempo y su contenido en frecuencia no permanece constante, sino que varía a lo largo del transitorio de arranque.

Como se ha mencionado anteriormente, para localizar las firmas asociadas a las distintas fallas que se pueden presentar en un motor, se han utilizado diferentes técnicas de análisis para la descomposición tiempo-frecuencia, permitiendo trazar la evolución de tales frecuencias en el tiempo. Un ejemplo de estas descomposiciones es la transformada de Fourier en tiempo corto (STFT, acrónimo de Short Time Fourier Transform).

En el trabajo realizado por Gandhi et al. [51] se presenta una revisión de técnicas que se utilizan para la detección de fallas en motores eléctricos, entre las técnicas que se mencionan se encuentra la STFT. Las ventajas de la utilización de la STFT son su fácil implementación y su simplicidad computacional; estas características de la STFT permiten obtener de forma rápida una idea del contenido espectral de la señal, lo cual se puede usar como punto de partida para el análisis con técnicas más complejas. Uno de sus inconvenientes es la elección de la ventana, la cual permanece constante para el análisis de la señal en todo el tiempo. Otro de los inconvenientes es el compromiso de la resolución en tiempo y en frecuencia; esto significa que, u obtenemos una buena resolución en frecuencia a costa de una pobre resolución en tiempo u obtenemos una buena resolución en tiempo a costa de una pobre resolución en frecuencia.

También en el trabajo realizado por Su et al. 52] se presenta la aplicación de la STFT para analizar señales cuasi-estacionarias y posteriormente utilizar esta información para entrenar redes neuronales. Las señales de vibración que se analizan se consideran cuasi-estacionarias debido a que las señales son considerablemente intermitentes. La detección de las fallas la hacen buscando cambios en el espectro de la señal de vibración. Las fallas que diagnostican son cortocircuitos en el devanado y desbalance en la alimentación. La aportación de este trabajo es que es posible detectar fallas eléctricas en el espectro de vibraciones además de 
que no es necesario conocer parámetros del motor. Sin embargo, uno de los inconvenientes es que las vibraciones en un motor se ven afectadas muy fácilmente por vibraciones externas, situación que provocaría que la metodología fallara en la clasificación de las averías.

En el trabajo realizado por Nandi et al. [53 se presenta otro caso de aplicación de la STFT para la detección de fallas en motores de inducción considerando los parámetros de la placa de características del motor en cuestión. En este trabajo se detecta la falla de excentricidad tanto estática como dinámica. Una de las aportaciones de este trabajo es que asocian los cambios en amplitud de los armónicos impares a la excentricidad estática y la excentricidad dinámica a armónicos intermedios. Algo que resulta interesante es que se use como indicador de falla cambios en los patrones alrededor de los armónicos espaciales.

En el trabajo realizado por Cabal-Yepez et al. [54] se presenta la implementación de la STFT y la transformada wavelet discreta en hardware reconfigurable de bajo costo, esto con el fin de monitorear equipos industriales, entre ellos motores eléctricos. Los autores de este trabajo aprovechan la relativa facilidad de implementación que ofrece la STFT, así como de la DWT para implementarlas en hardware. Este trabajo tiene la característica de que se obtiene un dispositivo que permite realizar prácticamente en línea, el análisis de señales de corriente, tensión y vibraciones. Si bien es cierto que la STFT y la DWT no son las herramientas de análisis más potentes si pueden brindar información importante acerca del funcionamiento del motor y toda la información que proporcionen seguramente servirá como fundamento para un análisis más complejo.

Otra de estas descomposiciones que permiten conocer el contenido de una señal en tiempo y en frecuencia, las cuales se han aplicado al diagnóstico de motores de inducción es la transformada wavelet discreta (DWT) [54]. La DWT ha mostrado que es efectiva para analizar señales no estacionarias. La DWT se calcula usando un conjunto de filtros pasa-bajas y pasa-altas, seguido de un submuestreo de la señal. Al aplicar la DWT se obtiene una descomposición de la señal en una serie componentes de baja y alta frecuencia. Esta descomposición nos puede dar información en el dominio del tiempo acerca de las frecuencias contenidas en la señal, además después de aplicar una transformación al dominio de la frecuencia a los componentes obtenidos se puede conocer de manera precisa el contenido espectral de los 
componentes obtenidos con la DWT.

En la investigación realizada por Riera-Guasp et al. [55] se presenta una metodología para diagnosticar la falla de barras del rotor. El método se enfoca en el estudio de una señal resultante de la descomposición con wavelets de la señal de corriente capturada durante el transitorio de arranque. Además, este trabajo muestra que una de las aproximaciones obtenidas con la DWT, prácticamente reproduce la evolución temporal del amónico lateral izquierdo, el nivel de descomposición en que se encuentre dicha señal dependerá de la tasa de muestreo, la similitud de la forma de esta señal con la forma de onda características del armónico izquierdo durante el transitorio es la evidencia de la falla en el rotor. En este trabajo se presenta una forma alternativa para detectar la falla de barras rotas analizando las descomposiciones que arroja la DWT.

En la investigación realizada por Cusido et al. [56] se propone una metodología de análisis basada en el espectrograma y la DWT. La metodología se aplica a la detección de barras del rotor rotas y bobinas en cortocircuito. La señal que se analiza en este trabajo es la corriente en estado estacionario, pero se introducen variaciones en la carga, haciendo que las señales capturadas sean no estacionarias. La propuesta de este trabajo es interesante desde el punto de vista de la combinación de técnicas para determinar la densidad espectral de potencia y la forma en que hacen el diagnóstico de la falla.

En el trabajo realizado por Panigrahy et al. [57] se presenta la implementación en FPGA de una técnica que conjunta la DWT y la FFT para la detección de barras rotas en motores de inducción. Para la detección de la falla se analizan señales de vibraciones, este trabajo es similar al mencionado en el párrafo anterior. Las ventajas que ofrece este trabajo en cuanto a lo ya realizado, mas allá de la metodología de diagnóstico es la implementación en FPGA (acrónimo de Field Programmable Gate Array).

En el trabajo realizado por Lee [58] se presenta un método de diagnóstico de fallas en el rotor y rodamientos para motores de inducción que se basa en la DWT y redes neuronales artificiales. En este trabajo las fallas son clasificadas con el uso de las redes neuronales utilizando directamente las descomposiciones que se obtienen con la DWT. La aportación de este trabajo es el uso de redes neuronales en conjunto con la DWT.

En el trabajo realizado por Ahamed et al. [59] se presenta una metodología de diagnóstico

UG - UVa

Paulo Antonio Delgado Arredondo 
para detectar barras rotas y desbalance mecánico en motores de inducción. Esta metodología propuesta utiliza la DWT como técnica de análisis de señales de corriente del transitorio de arranque. La detección de las fallas la obtienen midiendo la energía contenida en la cuarta, quinta y sexta descomposición, y determinan que cuando se presenta el desbalance la energía es mayor que cuando el motor está sano y cuando hay barras rotas la energía es menor que cuando el motor esta sano.

Rodriguez-Donate et al. [60 realizan una investigación para la identificación de fallas múltiples en un motor de inducción alimentado directamente de la línea de suministro, y basado en el análisis del transitorio de arranque mediante la aplicación de la DWT a señales de vibración. La aportación más importante de este trabajo es que pueden detectar fallas múltiples, esto es importante resaltarlo ya que generalmente los trabajos en cuanto a diagnostico se enfocan a fallas individuales.

En el trabajo realizado por Yahia et al. 61] también se presenta la utilización de la DWT en una metodología de diagnóstico para detectar fallas en motores de inducción. En este trabajo se detecta excentricidad en el motor a través del análisis de señales de corriente y tensión. Para obtener el diagnóstico del motor con esta metodología no es necesario conocer el deslizamiento del motor.

Otra de las técnicas de análisis tiempo-frecuencia es la transformada wavelet continua (CWT, acrónimo de Continuous Wavelet Transform) [62]. En el trabajo realizado por Saghafinia et al. en [63] y [64] se presenta una metodología para la detección de fallas en motores de inducción. Esta metodología además utiliza lógica difusa para la detección de barras rotas en motores de inducción. En esta metodología la CWT es utilizada para detectar las amplitudes y frecuencias de los componentes relacionados a las fallas y a los distintos niveles de carga. Esta información acerca de la amplitud y los valores de frecuencia de los componentes asociados a las fallas es la que se utiliza para entrenar el controlador de lógica difusa.

En el trabajo realizado por Das et al. [65] se presenta una metodología de diagnóstico para fallas en motores de inducción, el clasificador SVM (acrónimo de Support Vector Machine) se usa para caracterizar la falla de cortocircuito en el estátor usando las características de la falla obtenidas con la CWT.

UG - UVa

Paulo Antonio Delgado Arredondo 
Pilloni et al. 66 presentan un estudio de diferentes metodologías como la transformada rápida de Fourier (FFT), la transformada de Hilbert (HT), la DWT, la CWT y la distribución de Wigner-Ville (WVD) aplicada a la corriente del estátor de motores de inducción para la detección de fallas tanto en estado estacionario como en condiciones de operación transitorias.

En la investigación realizada por Feng et al. 67] se realiza una revisión detallada de métodos de análisis tiempo-frecuencia aplicados recientemente en el diagnóstico de fallas en máquinas. En este estudio se analizan las ventajas y desventajas del uso de los distintos métodos de análisis tiempo-frecuencia. Los métodos de análisis que se analizan van desde la STFT, la transformada wavelet, las distribuciones bilineales tiempo-frecuencia hasta métodos paramétricos y no paramétricos de análisis.

Otra técnica de análisis es la transformada Hilbert y Hilbert-Huang. En el trabajo realizado por Antonino [68 hacen una comparación entre la DWT y la transformada Hilbert-Huang aplicadas a corrientes capturadas durante el transitorio de arranque para detectar asimetrías en el rotor de motores de inducción. Al aplicar la transformada de Hilbert se obtiene una señal analítica, una característica importante de la señal analítica es que, si se obtiene su transformada de Fourier, los componentes para frecuencias negativas serán igual a cero, sin embargo, la parte positiva del espectro permanecerán inmutables y duplicara la componente de continua. La metodología Hilbert-Huang, realiza la descomposición de la señal en funciones de modo intrínseco por medio de la EMD y posteriormente se utiliza la transformada Hilbert para calcular la amplitud instantánea y la frecuencia instantánea de las funciones de modo intrínseco seleccionadas. Una de las ventajas de esta metodología es que no se involucran los conceptos de resolución en tiempo y resolución en frecuencia que tanto comprometen la eficiencia de técnicas convencionales de análisis tiempo-frecuencia, como la STFT.

En el trabajo realizado por Peng et al. [69 se analiza y se compara la eficiencia de la transformada Hilbert-Huang con métodos basados en wavelets y una mejora en la obtención de funciones de modo intrínseco. En este trabajo se utilizan wavelets para mejorar la eficiencia de la HHT, además de que se aplica una mejora para la obtención de las funciones de modo intrínseco en la EMD, la cual tiene el inconveniente del mezclado de modos además del 
rizado en los extremos de las funciones de modo intrínseco provocado por el reducido número de puntos disponibles en los extremos para calcular las envolventes.

Otra de las técnicas de descomposición tiempo-frecuencia y la cuál es una de las más empleadas en investigaciones relacionadas con el diagnostico de fallas en motores de inducción es la distribución de Wigner-Ville (WVD, acrónimo de Wirner-Ville Distribution) 66, 70 76]. Esta técnica de descomposición aporta la mejor resolución tiempo-frecuencia a costa de introducir términos cruzados los cuales son difíciles de identificar cuando se analizan señales con múltiples componentes espectrales.

En el trabajo realizado por Climente-Alarcon et al. [70] se analizan señales de corriente capturadas durante el transitorio de arranque de motores de inducción conectados directamente a la red eléctrica. Las fallas que diagnostican son asimetrías en el rotor y excentricidad mixta. La novedad de su trabajo se basa en el uso de filtros Notch avanzados para remover componentes de frecuencia constante presentes en las señales de corriente, esto con el fin de disminuir los términos de cruzados en la representación tiempo-frecuencia.

En el trabajo realizado por Refaat et al. [71] se analizan señales de corriente capturadas durante el funcionamiento en estado estable de motores de inducción conectados directamente a la red eléctrica. La falla que diagnostican son defectos en la pista externa de rodamientos. La metodología propuesta emplea la EMD para descomponer la señal en funciones de modo intrínseco, luego a estas funciones de modo intrínseco se les aplica la distribución WignerVille con el propósito de obtener su evolución tiempo-frecuencia. Posteriormente se analizan los patrones de las representaciones tiempo-frecuencia con las redes neuronales artificiales y a partir de esto se obtiene la condición del motor. Las funciones de modo intrínseco al ser monocomponentes presentarán menos términos cruzados después de obtener su representación tiempo-frecuencia con la WVD, esta es una de las aportaciones interesantes de este trabajo. Además, otro punto interesante de este trabajo es la unión y el aprovechamiento de las cualidades de distintas herramientas de análisis de señales.

En el trabajo realizado por Climente-Alarcon et al. [72] se analizan señales de corriente capturadas durante el transitorio de arranque de motores de inducción conectados directamente a la red eléctrica. Las fallas que se analizan en este trabajo son asimetrías y excentri- 
cidad en el rotor. La aportación principal de este trabajo es que, para obtener el diagnóstico analizan un mayor ancho de banda para buscar armónicos de alto orden relacionados a las fallas, el análisis de la señal en el transitorio de arranque lo hacen con ayuda de la WVD.

En el trabajo realizado por Climente-Alarcon et al. [73 se analizan señales de corriente capturadas durante el transitorio de arranque de motores de inducción conectados directamente a la red eléctrica. La falla que se analiza en este trabajo es la excentricidad mixta en el rotor. La aportación principal de este trabajo es la comprobación de la utilidad de tener la evolución en el transitorio de armónicos de alto orden relacionados con la excentricidad mixta, esto con el fin de diagnosticar el motor, complementando la información proporcionada por los armónicos de bajo orden. Para obtener la evolución de las frecuencias asociadas a la excentricidad mixta se utiliza la WVD.

En el trabajo realizado por Climente-Alarcon et al. [74] se analizan señales de corriente capturadas durante el transitorio de arranque de motores de inducción conectados directamente a la red eléctrica. La falla que se analiza en este trabajo son corto circuitos en el estátor. La aportación principal de este trabajo es diagnosticar la falla por medio del análisis de señales capturadas en el transitorio de arranque, para lograr esto se aplica la distribución Wigner-Ville y la distribución Choi-Williams.

En el trabajo realizado por Blödt et al. [75] se analizan señales de corriente capturadas durante el transitorio de arranque de motores de inducción alimentados con inversores. Las fallas que se analizan en este trabajo son oscilaciones en el par de la carga y desbalance en la carga. La aportación principal de este trabajo es diagnosticar la falla por medio del análisis de señales capturadas en el transitorio de arranque en motores alimentados por inversores, para lograr esto se aplica la distribución Wigner-Ville a las señales de corriente.

En la investigación realizada por Rajagopalan et al. [76] se analizan señales de corriente capturadas durante el funcionamiento transitorio de motores de corriente directa. Las fallas que se analizan en este trabajo son desbalance y excentricidad dinámica. La aportación principal de este trabajo es diagnosticar la falla por medio del análisis de señales no estacionarias, para lograr esto se aplica la distribución Wigner-Ville a las señales de corriente.

En otra investigación realizada por Rajagopalan et al. [77] se presenta un estudio de técni-

UG - UVa

Paulo Antonio Delgado Arredondo 
cas de descomposición tiempo-frecuencia cuadráticas. En este trabajo se analizan las características de las distribuciones tiempo-frecuencia CWD (acrónimo de Choi-Williams Distribution), la distribucion ZAM (acrónimo de Zhao-Atlas-Marks), la WVD, la SPWVD (acrónimo de Smoothed Pseudo-WVD), la distribución BJ (acrónimo de Born-Jordan), etcétera. Todas estas distribuciones se diferencian en sí, solo en un kernel o núcleo, ya que todas ellas pertenecen a la clase de Cohen, esta clase de distribuciones tiempo-frecuencia cumplen con las propiedades de invarianza ante desplazamientos temporales e invarianza ante desplazamientos frecuenciales. La elección del núcleo es de gran importancia ya que de ello dependerán de las características de la distribución tiempo-frecuencia.

Otra de las técnicas de análisis es la distribución de Choi-Williams, en esta distribución se consigue eliminar términos cruzados con un kernel, a costa de emporar la resolución tiempofrecuencia. En el trabajo realizado por Climente-Alarcon et al. 74 presentan la aplicación de la distribución Choi-Williams para analizar el transitorio de arranque de un motor de inducción.

Otra de las técnicas de analisis tiempo-frecuencia es la que se presenta en la investigación realizada por Yang et al. [78], en este trabajo se propone una transformación tiempofrecuencia nombrada SCT (acrónimo de Spline-kernelled Chirplet Transform). En esta técnica se introducen dos operadores, un operador de rotación en frecuencia y un operador de desplazamiento en frecuencia, esta técnica es muy eficiente particularmente para analizar señales altamente no lineales. Además, se presenta un algoritmo para estimar los parámetros del kernel en la SCT. La técnica es validada analizando señales de vibración. Los resultados que se obtienen con la SCT son mejores que los obtenidos con la CWT, la WVD y la STFT. Aunque esto tiene un precio computacional, aquí se presentan los resultados después de la primera iteración y los tiempos de cálculo resultan ser aproximadamente los mismos para la CWT y la WVD, sin embargo, para conseguir los mejores resultados con la SCT se muestra que son necesarias hasta seis iteraciones. A pesar de los tiempos de procesamiento, resulta interesante la aplicación de esta transformación en señales de corriente y de sonido, además de a otros tipos de fallas.

Por último, otra de las técnicas de descomposición tiempo-frecuencia que ha sido empleada

UG - UVa

Paulo Antonio Delgado Arredondo 
es la clasificación de señales múltiples (MUSIC). García-Pérez et al. 26] presentan un estudio de detección de fallas múltiples en un motor de inducción aplicando MUSIC a la corriente de un motor de inducción en estado estacionario; en este trabajo se utiliza un sensor de efecto Hall para medir señales de corriente de estátor y un sistema de adquisición de datos para adquirir la señal.

En otro trabajo realizado por García-Pérez et al. [37] se realiza una investigación de detección de fallas en un motor de inducción mediante el análisis de señales de sonido y vibración, en estado estacionario mediante la aplicación de MUSIC.

García-Pérez et al. [79] presentan un estudio experimental de la evolución en el plano tiempo-frecuencia de las características de la corriente del estátor durante el transitorio de arranque, cuando la barra se rompe parcialmente en un motor de inducción alimentado directamente de la red eléctrica. Este análisis tiempo-frecuencia se hace mediante la aplicación de MUSIC a la señal del transitorio de arranque.

La mayoría de estas técnicas se han utilizado para analizar las señales de corriente del estátor. En el caso de las señales de vibración o sonido, no se han realizado numerosas investigaciones. Solo se han aplicado técnicas tales como MUSIC en el trabajo realizado por Garcia-Perez [37]. También se encuentran algunos otros trabajos donde se utilizan la transformada rápida de Fourier (FFT) [36], la ZAM [34] y la DWT [35].

Después de analizar las técnicas de detección, se puede ver que todas son eficientes en el diagnóstico de fallas en motores de inducción, a pesar de esto también tienen algunos inconvenientes. Si bien es cierto que la aplicación de una u otra dependerá del tipo de señal que se pretende analizar, es decir, si lo que se quiere analizar es la señal de corriente del estator durante el funcionamiento en estado estacionario, sin lugar a dudas la transformada de Fourier será la mejor opción. Sin embargo, si lo que se pretende es analizar la corriente en el transitorio de arranque, se tendrán que tomar en cuenta las características de las distintas técnicas, por ejemplo, una opción puede ser la WVD, esta distribución ofrecerá la mejor resolución posible para un análisis tiempo-frecuencia, no obstante, esta representación también arrojara términos cruzados, los cuales si se desconocen las características de la señal analizada llevarán a errores de interpretación. Otra opción interesante sería el uso de la 
STFT, a pesar de que en esta representación se da un compromiso entre resolución en tiempo y resolución en frecuencia, tiene la ventaja de su fácil implementación e interpretación de los resultados, asimismo sirve para dar una idea de lo que se debe esperar al hacer un análisis más complejo. Caso similar a las señales de corriente sucede con las señales de vibración, estas señales al tener una gran cantidad de ruido de fondo, la mejor opción para un análisis en transitorio o en estado estable, será una técnica que permita minimizar los efectos del ruido. La misma situación se da en señales de sonido, que al igual que en vibraciones presentan una gran cantidad de ruido de fondo. Una de las técnicas que más llama la atención es el algoritmo MUSIC, con este algoritmo se puede obtener una representación con alta resolución en tiempo y en frecuencia, además de una eliminación del ruido presente en la señal, a pesar de estas ventajas, posee el inconveniente de que las amplitudes de los componentes del espectro y de la señal no tienen una relación directa. Esta técnica también presenta frecuencias espurias, estas frecuencias en realidad no están presentes en la señal y surgen por una mala elección de parámetros en el algoritmo.

\subsubsection{Técnicas de diagnóstico}

Algunas de las técnicas de análisis vistas en la sección anterior pueden trabajar conjuntamente con clasificadores de inteligencia artificial (AI) para la toma de decisiones sobre los componentes o firmas que están presentes en las señales capturadas en los motores, para identificar las fallas y su gravedad $80-82$. Estos clasificadores de AI incluyen las redes neuronales artificiales (ANN). En el trabajo que presenta $\mathrm{Su}$ et al. [52 se proporciona el desarrollo de un sistema de detección de fallas eléctricas en línea que utiliza modelos de redes neuronales aplicados a señales de vibración de motores de inducción. La STFT se utiliza para procesar las señales de vibración y obtener el espectro de la señal, de manera que el modelo de red neuronal pueda ser entrenado. En el trabajo de Lee [58] se propone un método de diagnóstico de fallas para motores de inducción basado en la DWT y ANN. El método de diagnóstico de fallas propuesto consiste en la parte de preprocesamiento de datos mediante el análisis en frecuencia de la señal de vibración y el clasificador de fallas para el aislamiento de fallas. La parte de preprocesamiento de datos que descompone las señales en las distintas 
bandas de frecuencia por medio de la DWT es muy importante para mejorar el rendimiento del diagnóstico de fallas. En este trabajo se consideran tanto fallas en el rotor como en los rodamientos.

Otra de las técnicas de AI que se han conjuntado con técnicas de análisis tiempo-frecuencia es la lógica difusa. En sus trabajos 63, 64, Saghafinia et al. presentan una metodología que usa la lógica difusa y una wavelet adaptativa para el diagnóstico en línea de barras del rotor rotas en motores de inducción de jaula de ardilla. Estas señales se analizan a través de la CWT adaptativa para detectar las amplitudes y componentes de frecuencia correspondientes a diferentes condiciones de fallo y carga. En esta metodología los coeficientes de la CWT son adaptados en base a la amplitud de los armónicos, estos coeficiente son los que se utilizan en el controlador de lógica difusa. Estas amplitudes y frecuencias se aplican para entrenar un controlador de lógica difusa (FLC) en la simulación. A continuación, la CWT adaptable y el FLC entrenado se aplican para detectar la condición de falla del motor.

Otras técnicas de AI que han sido implementadas son las redes neuronales difusas y algoritmos genéticos. En el estudio del estado de la técnica que presenta Bindu y Vinod [83], se revisan las tendencias actuales en el diagnóstico interno de fallas de máquinas de inducción e identifica futuras opciones de investigación. En el trabajo realizado por Zhang et al. [84 se presenta una revisión exhaustiva de los métodos de monitoreo y diagnóstico de las condiciones existentes en las cinco áreas siguientes: protección térmica y estimación de la temperatura, supervisión del aislamiento del estátor y detección de fallas, detección de fallas en los rodamientos, detección de barras rotas y detección de excentricidad. Para cada una de las cinco áreas de estudio mencionadas anteriormente, se discuten las características relacionadas de los motores de media tensión; la eficacia de los métodos existentes se discute en términos de su robustez, precisión y complejidad de implementación. También se presentan recomendaciones para la futura investigación en estas áreas. 


\subsection{Planteamiento del problema}

En la actualidad se presentan algunos inconvenientes en métodos utilizados para detectar algún tipo de falla en motores eléctricos. Algunas metodologías son invasivas o requieren de instalaciones especiales y complejas, algunas son susceptibles al tipo de alimentación del motor, es decir, si este es alimentado directamente de la red eléctrica o si es a través de un variador de velocidad.

Si bien es cierto que existen diversos métodos para determinar las condiciones de un motor eléctrico, es necesario desarrollar una metodología de diagnóstico de fallas que pueda aplicarse a señales de sonido provenientes de un motor, además de una metodología aplicable a señales de vibración que sea capaz de detectar fallas durante el transitorio de arranque de un motor. Estas metodologías deberían ser capaces de determinar fiablemente fallas tempranas en los componentes de un motor eléctrico, antes de que una intervención humana o automática sea necesaria.

\subsubsection{Hipótesis}

Es posible diagnosticar distintas condiciones de falla en un motor eléctrico de corriente alterna de rotor tipo jaula de ardilla por medio del desarrollo de una metodología que permita mejorar el diagnóstico de las condiciones de este tipo de motores, esta metodología hará el procesamiento digital de señales de vibraciones y sonido, durante el funcionamiento en estado estable y el transitorio de arranque, usando técnicas de análisis espectral.

\subsubsection{Justificación}

En la actualidad, el motor de inducción de rotor tipo jaula de ardilla es el más usado en aplicaciones industriales. El desarrollo continuo de investigaciones en este campo es necesario para mejorar los diagnósticos y así evitar pérdidas en la productividad, debidas a paros no previstos para dar mantenimiento a causa de alguna falla que pudiera haber sido detectada con anterioridad. En este hecho radica la importancia de la detección y diagnóstico de fallas 
de forma temprana y fiable. Aunque en la actualidad existen numerosas metodologías para el monitoreo y diagnóstico; en los centros de investigación la gran mayoría de las investigaciones se basan en el análisis de corrientes del estátor y unas cuantas en el análisis de señales de vibración. En cambio, el análisis de vibraciones en la industria es usado como una vía rápida para diagnosticar fallas mecánicas en el estacionario del motor. Respecto a señales de vibración en el transitorio de arranque del motor, poco se ha hecho y en cuanto al análisis de señales de sonido prácticamente nada. De esta forma surge la oportunidad del análisis de señales de vibraciones durante el transitorio de arranque, dicho análisis puede proporcionar información relevante de la condición del motor, sobre todo cuando se presentan fallas mecánicas, así mismo puede complementar un diagnóstico realizado con el análisis de corrientes. Aunado a lo anterior, la búsqueda de técnicas alternativas como el sonido para realizar la detección y el diagnóstico de fallas es un área de estudio desconocida la cual promete buenos resultados.

\subsection{Objetivos}

Objetivo general. Formular una metodología de análisis espectral para la detección y diagnóstico de fallas en motores de inducción de rotor tipo jaula de ardilla, analizando señales de vibración durante el transitorio de arranque y señales de sonido durante el estado estable.

Objetivos específicos. Los objetivos específicos contemplados en el presente trabajo de tesis se enlistan a continuación:

- Diseñar el banco de pruebas con un motor de inducción de rotor tipo jaula de ardilla, para obtener las señales con las distintas condiciones de falla.

- Generar las fallas en los componentes del motor que se analizarán.

- Obtener las distintas señales de vibraciones y sonido de cada condición de falla y de un motor sano.

- Crear una base de datos con las señales obtenidas.

$\mathrm{UG}-\mathrm{UVa}$

Paulo Antonio Delgado Arredondo 
- Validar las herramientas de análisis espectral propuestas.

- Realizar el procesamiento de las señales de vibraciones y de sonido aplicando las herramientas de análisis espectral.

- A partir de lo obtenido con el procesamiento de las señales, analizar la eficiencia y la aplicabilidad de la metodología propuesta.

\subsection{Organización de la tesis}

El presente libro de tesis está compuesto por seis capítulos. Organizados de tal forma que se lleva un orden natural en la presentación de la información para facilitar al lector su comprensión. En el Capítulo 2 se presenta la teoría necesaria para comprender la constitución de los motores de inducción, su principio de funcionamiento, las fallas que comúnmente se presentan en ellos y que se analizan en el presente trabajo, además de los espectros teóricos de las fallas. En el Capítulo 3 se presenta el marco teórico referente al análisis espectral. En este capítulo se describen los fundamentos del análisis espectral de señales, tomando como punto de partida la transformada de Fourier. En el Capítulo 4 se detalla la puesta experimental del banco de pruebas, la generación de las fallas, el procesamiento de las señales, se proporciona la validación de las técnicas utilizadas y además de la metodología necesaria para la obtención del diagnóstico de los motores. En el Capítulo 5 se describen los diferentes casos de estudio considerados en este trabajo, la presentación de resultados, la validación de los resultados y un análisis y discusión exhaustivo de los resultados. Por último, en el Capítulo 6 se dan las conclusiones del trabajo de tesis poniendo en contraste los objetivos con los resultados obtenidos, así como el trabajo que se podría realizar a futuro.

UG - UVa

Paulo Antonio Delgado Arredondo 


\section{Capítulo 2}

\section{Motores de inducción}

Los motores eléctricos son de gran importancia en el mundo industrial moderno. Son una de las máquinas más usadas: más del $40 \%$ de la electricidad consumida en todo el mundo se utiliza para alimentar motores eléctricos [1]. También se considera que aproximadamente la mitad de la electricidad consumida por la industria en los Estados Unidos es utilizada por motores de inducción, donde el $89 \%$ de los motores eléctricos son motores asíncronos [85]. Las aplicaciones son variadas y las ventajas de su uso son numerosas. Además de su uso en la industria también están presentes en varios modos de transporte y en los hogares. Como resultado, son elementos básicos en el mundo moderno y de este hecho surge la necesidad de un diagnóstico rápido y preciso de las fallas para anticipar el paro en los procesos donde se utilizan. Además basado en su amplia presencia, la eficiencia de los motores eléctricos se convierte en un tema de interés global.

Un motor eléctrico es una máquina que transforma energía eléctrica en energía mecánica por medio de interacciones electromagnéticas. Los motores eléctricos son reversibles, es decir pueden transformar energía mecánica en energía eléctrica funcionando como generadores. Pueden funcionar conectados a una red de suministro eléctrico o a baterías, como en determinadas aplicaciones de tracción. Los motores de corriente alterna y los de corriente continua se basan en el mismo principio de funcionamiento, el cual establece que si un conductor por el que circula una corriente eléctrica se encuentra dentro de la acción de un campo magnético, 
éste tiende a desplazarse perpendicularmente a las líneas de acción del campo magnético. De esta forma, se produce el movimiento en un motor eléctrico y este entrega potencia mecánica.

El motor eléctrico, en diversas circunstancias, presenta muchas ventajas respecto a los motores de combustión:

- A igual potencia, su tamaño y peso son más reducidos.

- Se pueden construir de cualquier tamaño.

- Tiene un par de giro elevado y, según el tipo de motor, prácticamente constante a distintas velocidades.

- Su rendimiento es muy elevado, aumentando a medida que se incrementa la potencia útil de la máquina.

Este tipo de motores además no emite contaminantes directamente, aunque en la generación de energía eléctrica de la mayoría de las redes de suministro sí se pueden emitir gases contaminantes y de efecto invernadero en las centrales de producción eléctrica.

\subsection{Partes principales}

Un motor de inducción (figura 2.1) esta compuesto principalmente por un rotor, el cual es la parte rotatoria, y un estátor que constituye la parte estacionaria, en la cuál están las bobinas inductoras, la carcasa y una variedad de elementos auxiliares tales como escudos, bornera, entre otros, que son necesarios para el funcionamiento del motor y dependen mas del tipo y aplicación del mismo.

\section{Estátor}

El estátor (figura 2.2) es la parte fija de una máquina rotativa y uno de los dos elementos fundamentales para la transmisión de potencia. Aloja el circuito inductor, normalmente 


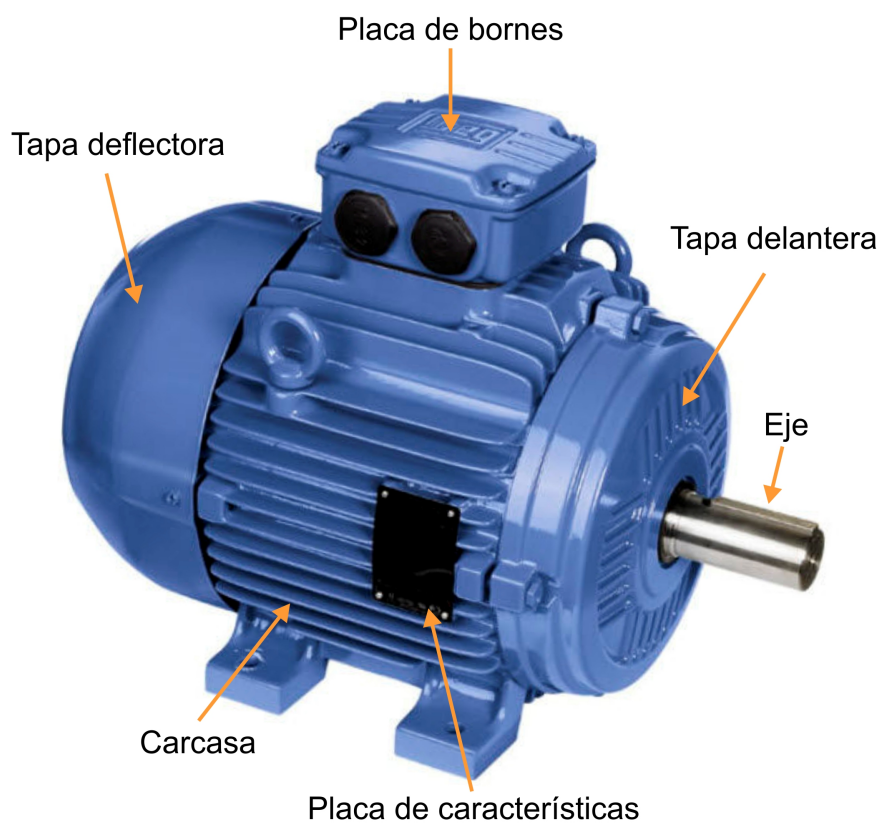

Figura 2.1: Partes principales.

trifásico y distribuido a lo largo de la periferia ranurada del circuito magnético. Este se construye por apilamiento de chapas magnéticas. Para disminuir las pérdidas de energía en el circuito magnético, estas chapas son de espesor muy reducido y están eléctricamente aisladas entre sí. También suelen ser de acero al silicio, con una menor conductividad eléctrica. De esta forma se reducen las pérdidas por histéresis y por corriente de Foucault.

\section{Rotor}

El rotor (figura 2.4) es el componente que gira o rota en una máquina eléctrica. Está formado por un eje que soporta un juego de bobinas arrolladas sobre un núcleo magnético o una serie de barras con dos anillos que las cortocircuitan. Gira dentro de un campo magnético creado por las corrientes, ya sean monofásicas o trifásicas, equilibradas que circulan por el circuito del estátor. El circuito magnético del rotor también se construye, como el estátor, mediante un apilamiento de chapas magnéticas ranuradas. 


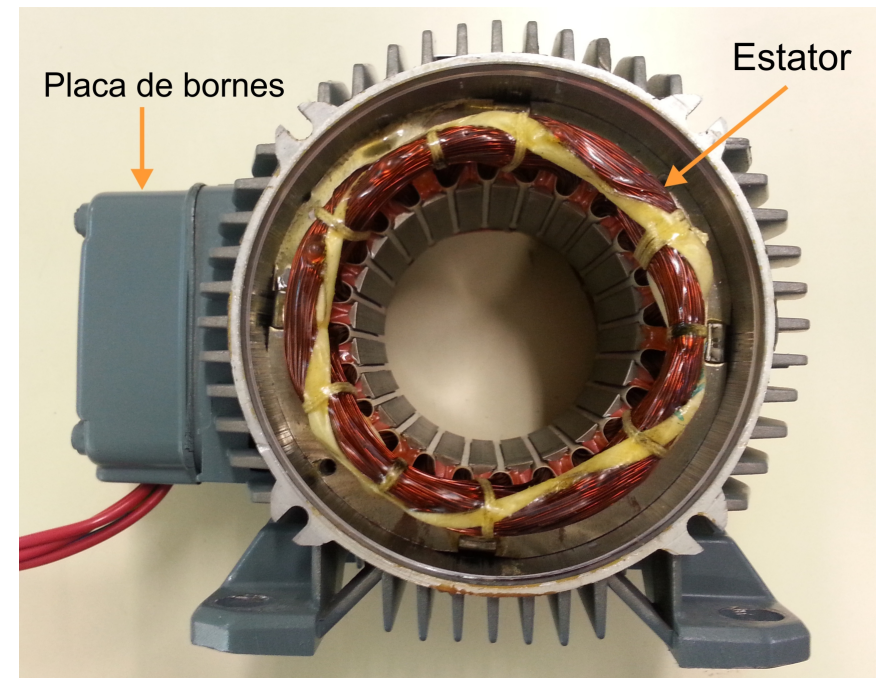

Figura 2.2: Estátor.

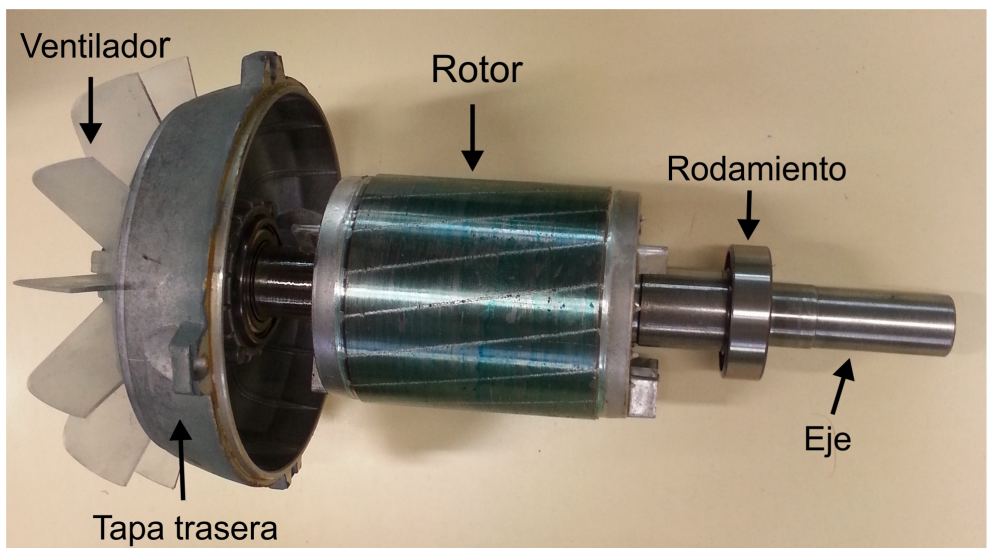

Figura 2.3: Rotor.

Constructivamente y a nivel industrial hay dos tipos de rotores. Uno de ellos, que está en desuso o se emplea en aplicaciones muy específicas, es el rotor de tipo devanado. El segundo y de uso más generalizado es el denominado rotor de jaula de ardilla (figura 2.4, figura 2.5). En este grupo se distinguen otros dos tipos. En baja potencia, se suelen emplear los rotores de aluminio inyectado. El circuito eléctrico se construye mediante la inyección de aluminio 
fundido dentro de un molde donde se encuentra el circuito magnético. El rotor, formado por unas barras y los anillos de cortocircuito, es de una sola pieza. Actualmente, también existen motores de jaula de cobre inyectado, que presentan una mayor eficiencia. Cuando se produce un fallo en este tipo de rotores, al no ser destructivo el fallo, el motor se sigue utilizando a pesar de trabajar con una menor eficiencia, hasta que el rotor es descartado, puesto que no son reparables.

El segundo tipo de rotor es el construido con barras de cobre, de sección rectangular, que se introducen en las ranuras. Estas barras se sueldan en sus extremos a los anillos de cortocircuito, también de cobre. Estas uniones son el punto más débil de la jaula. Estos rotores no son tan económicos como los rotores de aluminio inyectado, su fallo es destructivo y no se pueden seguir utilizando, por lo tanto, se deben reparar.

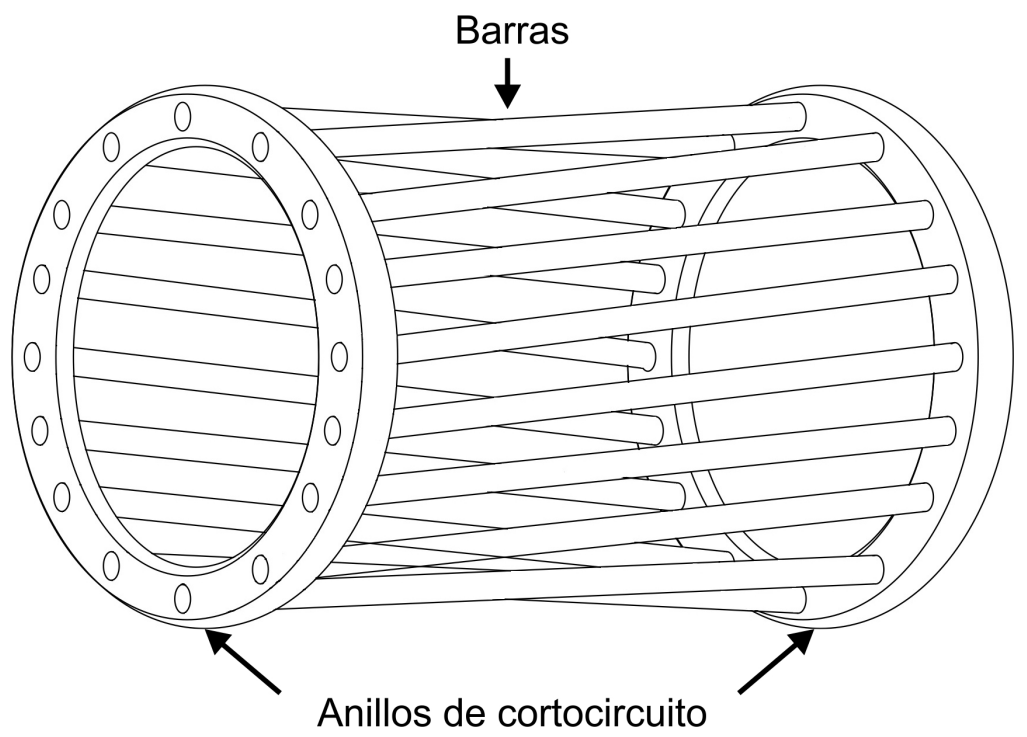

Figura 2.4: Jaula de ardilla.

El rotor gira dentro del estátor, a una velocidad inferior a la velocidad del campo magnético giratorio producido por el primero. La diferencia entre la velocidad del campo magnético del estátor y la velocidad de giro del rotor se llama deslizamiento y aumenta con la carga. 


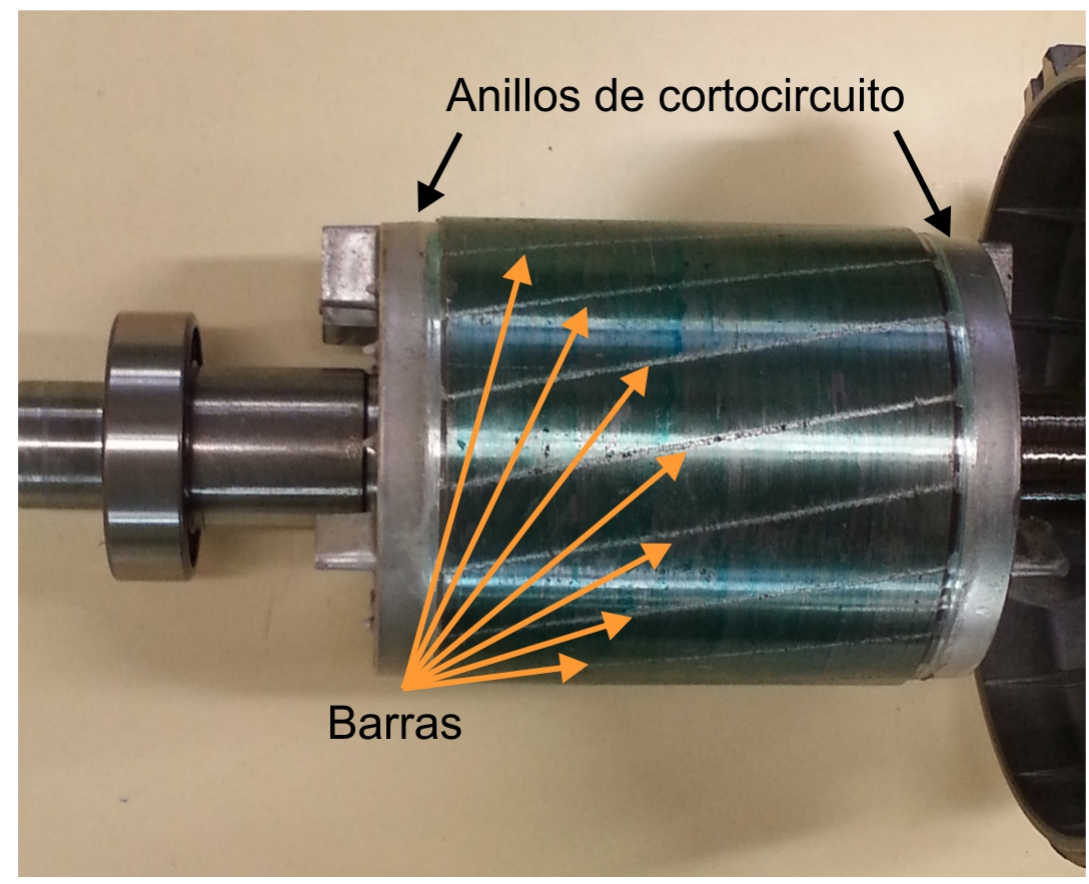

Figura 2.5: Jaula de ardilla.

A menudo, los conductores se inclinan levemente a lo largo de la longitud del rotor para reducir ruido y para reducir el par resistente de reluctancia que es debido a las interacciones con las ranuras del estátor. El número de barras en la jaula de la ardilla se determina según las corrientes inducidas en las bobinas del estátor y por lo tanto según la corriente a través de ellas. Las construcciones que ofrecen menos problemas emplean números primos de barras.

\subsection{Principio de funcionamiento}

Según el teorema de Ferraris, cuando circula un sistema de corrientes trifásicas equilibradas por las bobinas del estátor, se induce un campo magnético de módulo constante y giratorio de velocidad de giro también constante que atraviesa el entrehierro (espacio de aire entre rotor y estátor) y que concatena al rotor. La velocidad a la que gira este campo se cono- 
ce como velocidad de sincronismo. Ésta depende de la frecuencia de la fuente de alimentación y del número de polos magnéticos de la máquina. Se puede calcular como sigue:

$$
n_{\text {sinc }}=\frac{120 f_{s}}{P}
$$

donde $f_{s}$ es la frecuencia de la fuente de alimentación dada en $\mathrm{Hz}, P$ es el número de polos del motor y $n_{\text {sinc }}$ es la velocidad de rotación del campo magnético o velocidad de sincronismo (en r.p.m).

Este campo magnético genera un flujo variable en las fases del rotor e inducirá una fem en su circuito eléctrico según la Ley de Inducción de Faraday.

$$
e=-N \frac{d \phi}{d t}
$$

donde $e$ es la fuerza electromotriz, $N$ es el numero de vueltas y $\frac{d \phi}{d t}$ es la tasa de variación temporal de flujo magnético. Como el circuito eléctrico del rotor está cerrado, esta fuerza electromotriz inducida produce la circulación de corrientes. La interacción de estas corrientes con el campo magnético giratorio produce unas fuerzas sobre los conductores del rotor. Este campo de fuerzas produce un par que hace girar al rotor en la misma dirección que el campo magnético giratorio. El rotor nunca alcanzará la velocidad de sincronismo, porque en caso contrario la fem inducida sería nula.

Además, cabe recalcar que, durante el funcionamiento de un motor de inducción en régimen estacionario, los campos magnéticos generados en el rotor y el estátor, $\mathbf{B}_{R} \mathbf{y} \mathbf{B}_{S}$ (figura 2.6), rotan simultáneamente a velocidad síncrona $n_{\text {sinc }}$, mientras que el rotor siempre girara a una velocidad menor.

Normalmente se utiliza la ecuación 2.1 para determinar la velocidad del campo magnético del estátor o velocidad de sincronía y, el resultado se expresa en revoluciones por minuto en lugar de radianes por segundo. Sin embargo, el rotor gira más despacio a una velocidad cercana a la de sincronismo. Para medir la relación entre la velocidad de giro del rotor y la velocidad del campo magnético se define la velocidad del deslizamiento como:

$$
n_{\text {des }}=n_{\text {sinc }}-n_{m}
$$




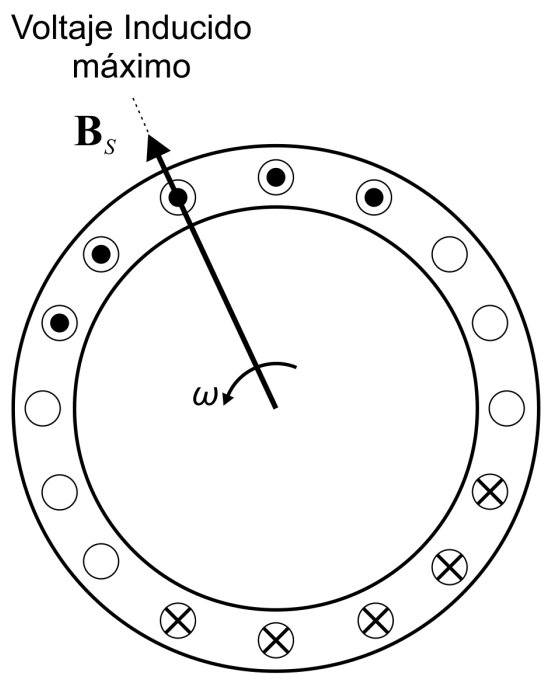

a)

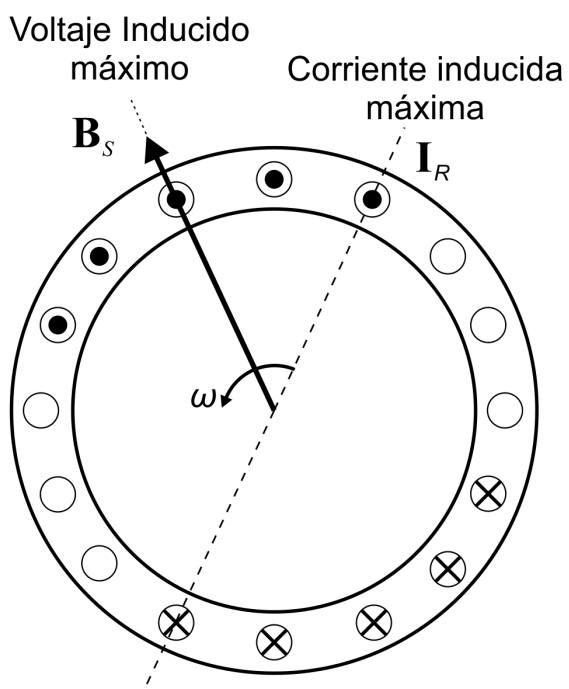

b)

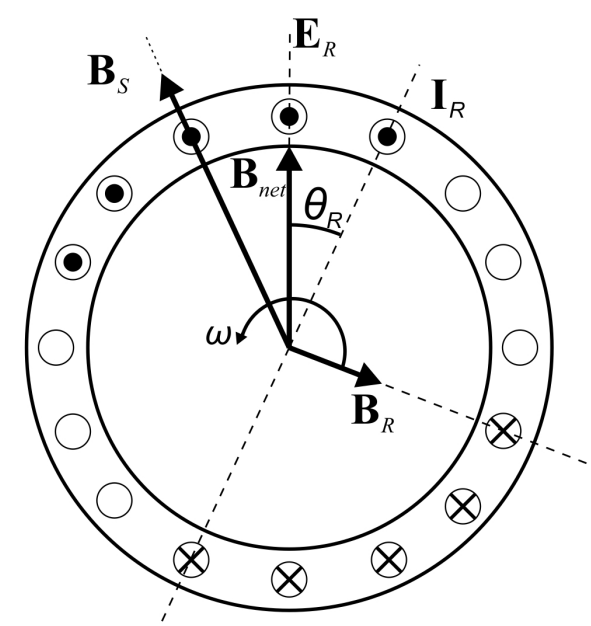

c)

Figura 2.6: Desarrollo del par inducido en un motor de inducción.

en la ecuación 2.3, $n_{\text {des }}$ representa la velocidad de deslizamiento, $n_{\text {sinc }}$ se refiere a la velocidad de sincronismo y $n_{m}$ a la velocidad en el eje del rotor.

Llamamos deslizamiento a la velocidad relativa expresada sobre una base por unidad o 
expresada en porcentaje. El deslizamiento esta definido por:

$$
\begin{gathered}
s=\frac{n_{\text {sinc }}-n_{m}}{n_{\text {sinc }}} \\
\% s=\frac{n_{\text {sinc }}-n_{m}}{n_{\text {sinc }}} \times 100 \%
\end{gathered}
$$

La ecuación 2.4 también puede expresarse en términos de la velocidad angular(radianes por segundo) como:

$$
s=\frac{\omega_{\sin c}-\omega_{m}}{\omega_{\sin c}}
$$

cuanto mayor sea el deslizamiento, mayor será la velocidad con que el campo cortará las espiras del rotor y, por consiguiente, resulta una mayor corriente inducida.

\subsection{Fallas en motores de inducción}

Los motores de inducción son componentes críticos para la mayoría de las industrias y su amplia presencia en el mundo moderno los hace indispensables. Las fallas en los motores de inducción pueden ocurrir en cualquiera de sus componentes, por ejemplo, en el estátor, el rotor y los rodamientos. De hecho, el $38 \%$ de las fallas se dan en el estátor, el $10 \%$ ocurren en el rotor y alrededor del $40 \%$ representan fallas mecánicas relacionadas con los rodamientos y el restante $12 \%$ pertenece a fallas en otros componentes [24].

\subsubsection{Barras rotas}

La detección de barras rotas en el rotor es una de las principales fallas a la cual se han enfocado muchos trabajos de investigación, debido a que el motor continua trabajando aparentemente en buenas condiciones sin mostrar cambios evidentes originados por la falla [4]. Sin embargo, esta falla provoca un incremento en el consumo de corriente y disminución del tiempo de vida de la máquina, esto debido a que se puede dañar el estátor, en cuyo caso el motor es irreparable y se debe reemplazar por otro. Además de los daños en el motor, se generan vibraciones las cuales afectan a componentes externos conectados al motor, como lo

UG - UVa

Paulo Antonio Delgado Arredondo 
son: acoplamientos, bandas de transmisión, engranes, etc.

En las barras del rotor de un motor de inducción, se sabe que corrientes simétricas circulando por las barras de un rotor simétrico inducen un campo magnético rotativo resultante hacia adelante; este campo magnético gira a la velocidad de sincronismo cuando las barras del rotor están sanas. Al presentarse la ruptura de una barra, genera asimetrías en el rotor, entonces como resultado, además del campo a la velocidad de sincronismo, habrá un campo magnético rotativo hacia atrás a la velocidad del deslizamiento $s f_{s}$ con respecto al rotor. Las interacciones del campo magnético rotativo inverso con el campo magnético del estator generan oscilaciones en el torque y en la velocidad, y la frecuencia de estas oscilaciones es $2 s f_{s}$, donde esta oscilación actúa como moduladora de frecuencia en la frecuencia de rotación y la frecuencia de falla $f_{B B}$ aparece alrededor de $f_{r}$ en el espectro de vibraciones [86]:

$$
f_{B B}=k f_{r} \pm 2 n s f_{s}
$$

donde $f_{s}$ es la frecuencia de la alimentación, $s$ es el deslizamiento del motor, $f_{r}$ es la velocidad del rotor en Hz, y $k$ y $n$ son enteros positivos. En el caso del transitorio de arranque se maximizan las componentes frecuenciales de la señal en comparación con el estado estable.

\subsubsection{Rodamientos}

Algunos autores proporcionan una revisión de las causas y las frecuencias en el espectro de vibraciones esperadas debido a defectos en rodamientos de bolas [87]. Una gran variedad de frecuencias asociadas con la rotación del rotor se puede obtener a partir de la geometría del rodamiento, como las frecuencias asociadas al paso de los balines del rodamiento ya sea en la pista externa o en la pista interna, frecuencias asociadas a la rotación de la jaula y frecuencias asociadas al giro de los balines.

Cuando se presenta un defecto en la pista externa del rodamiento, este causará un impulso cada vez que un balín esté en contacto con él. La velocidad de rotación del rotor $f_{r}$ es la velocidad a la cual la pista interna del rodamiento gira, la cual es la frecuencia de rotación 
del eje. La carga radial única se transforma en una carga distribuida porque el anillo interior está en contacto con más de una bola durante la rotación. Sin embargo, para la condición de fallo de cojinete cuando un defecto en una superficie de un cojinete golpea una superficie complementaria, se produce un impulso que excita resonancias en el sistema. El sistema mecánico es simétrico respecto a la línea de la carga aplicada. A medida que el rodamiento gira, los impactos producidos por el defecto en la pista externa del rodamiento ocurren a la frecuencia $f_{B P O F}$ dada por [88]:

$$
f_{B P O F}=\frac{N_{B}}{2} f_{r}\left(1-\frac{D_{b}}{D_{c}} \cos \theta\right)
$$

donde $\theta$ es el ángulo de contacto entre la superficie y el balín, $D_{c}$ es el diámetro del rodamiento, $D_{b}$ es el diámetro del balín y $N_{B}$ es el número de balines del rodamiento.

\subsubsection{Desbalance}

El desbalance mecánico en un motor de inducción envuelve la estructura completa del rotor, la cual está construida de múltiples componentes que incluyen el eje, las laminaciones del rotor, las barras del rotor, conectores, anillos de retención y el ventilador. Todos estos elementos deben ser diseñados y fabricados para un ensamblaje final que brinde un balance mecánico estable y preciso. Cuando un motor está apropiadamente balanceado y alineado, la amplitud de la frecuencia, asociada al desbalance, apenas cambia y permanece limitada en un cierto nivel.

Sin embargo, cuando el motor está desbalanceado mecánicamente, se presentan pequeñas vibraciones radiales en la estructura del rotor. El nivel de vibraciones toma su máximo cuando la velocidad de rotación es igual a la frecuencia natural del sistema definida como $\omega_{0}=\sqrt{k / m}$; donde $k$ es el factor de rigidez y $m$ es la masa de desbalance [89,90]. Además, esta señal vibratoria oscila a la frecuencia de rotación del rotor, estas vibraciones se incrementan, la amplitud de la frecuencia de rotación aumenta y lo mismo sucede con sus armónicos [91]. El análisis de señales de vibración puede proporcionar una rápida y fácil forma de extraer información 
que permita el diagnostico acerca de la presencia de desbalance en un motor de inducción.

Normalmente, el fabricante del motor proporciona la velocidad nominal de operación del motor, generalmente expresada en revoluciones por minuto. En motores asíncronos, esta velocidad es ligeramente menor que la velocidad de sincronismo, $n_{\text {sinc }}$, que está relacionada con la frecuencia de la alimentación $f_{s}$ como se expresa en la ecuación 2.1. 


\section{Capítulo 3}

\section{Análisis espectral}

\subsection{Señales no estacionarias}

Antes de hablar acerca de no estacionariedad, definiremos lo que se llama estacionario. Una señal determinística se dice que es estacionaria si esta puede ser representada como una suma discreta de sinusoides con amplitud y frecuencia instantáneas constantes:

$$
\begin{gathered}
\text { Para una señal real } \\
x(t)=\sum_{k \in \mathbb{N}} A_{k} \cos \left[2 \pi f_{k} t+\phi_{k}\right] \\
\text { Para una señal compleja } \\
x(t)=\sum_{k \in \mathbb{N}} A_{k} \exp \left[j\left(2 \pi f_{k} t+\phi_{k}\right)\right]
\end{gathered}
$$

Una señal no estacionaria se define como aquella en la cual su contenido en frecuencia va cambiando con el tiempo, esto quiere decir que no permanece constante, por lo tanto, para conocer el contenido espectral de este tipo de señales es necesaria una representación gráfica en la que se muestre simultáneamente el contenido espectral y el tiempo. 


\subsection{Representación temporal y frecuencial}

La representación temporal es comúnmente la primera y la más natural descripción de una señal, esto debido a que la mayoría de todas las señales físicas son obtenidas en función del tiempo. La representación frecuencial se obtiene de la transformada de Fourier, definida en su forma bilateral y en tiempo continuo por la siguiente ecuación [2]:

$$
X(f)=\int_{-\infty}^{+\infty} x(t) e^{-j \omega t} d t
$$

donde $X(f)$ es la transformada de Fourier de la señal continua $x(t)$ a la frecuencia $\omega=2 \pi f$. Es también una forma muy poderosa para describir una señal, esto porque el concepto de frecuencia esta compartido por muchas disciplinas (física, astronomía, economía, ingeniería, etc.) en los cuales ocurren eventos periódicos. Pero si vemos más cuidadosamente el espectro $X(f)$, éste puede ser visto como una función de coeficientes obtenidos por la expansión de la señal $x(t)$ en una familia de curvas, $e^{j 2 \pi f t}$, para las cuales no es posible determinar los instantes en los que ocurren en el tiempo. Por ende, el espectro esencialmente nos dice qué frecuencias están contenidas en la señal, así como sus amplitudes y fases, pero no nos dice en qué tiempo ocurren estas frecuencias [2].

\subsection{Localización y el principio de Heisenberg-Gabor}

Una forma simple de caracterizar una señal simultáneamente en tiempo y en frecuencia es considerar sus localizaciones promedio y dispersiones en cada representación. Esto puede ser obtenido considerando $|x(t)|^{2}$ y $|X(f)|^{2}$ como distribuciones de probabilidad, y buscando sus valores medios y desviaciones estándar 92$]$ :

$$
\begin{aligned}
& t_{m}=\frac{1}{E_{x}} \int_{-\infty}^{+\infty} t|x(t)|^{2} d t \quad \text { tiempo promedio } \\
& f_{m}=\frac{2 \pi}{E_{x}} \int_{-\infty}^{+\infty} f|X(j f)|^{2} d f \quad \text { frecuencia promedio } \\
& T^{2}=\frac{1}{E_{x}} \int_{-\infty}^{+\infty}\left(t-t_{m}\right)^{2}|x(t)|^{2} d t \quad \text { tiempo de dispersión }
\end{aligned}
$$




$$
B^{2}=\frac{4 \pi^{2}}{E_{x}} \int_{-\infty}^{+\infty}\left(f-f_{m}\right)^{2}|X(j f)|^{2} d f \quad \text { frecuencia de dispersión }
$$

donde $E_{x}$ es la energía de la señal, asumiendo que es finita, es decir:

$$
E_{x}=\int_{-\infty}^{+\infty}|x(t)|^{2} d t<+\infty
$$

Entonces, como resultado de las expresiones anteriores, una señal puede ser caracterizada en el plano tiempo-frecuencia por su posición promedio $\left(t_{m}, f_{m}\right)$ y un dominio de localización de energía principal cuya área es proporcional al producto tiempo-ancho de banda (TXB).

\subsection{Frecuencia instantánea y retardo de grupo}

Otra de las formas de describir la evolución frecuencial de una señal a lo largo del tiempo y que para señales con determinadas propiedades puede ser válida para su caracterización, es a través de la frecuencia instantánea y el retardo de grupo. Éstos vienen definidos por la derivada en el tiempo de la fase de la señal y la derivada en la frecuencia de la fase de la transformada de Fourier de la señal, respectivamente [93]:

$$
\begin{gathered}
\varphi^{\prime}(t)=\frac{1}{2 \pi} \frac{\operatorname{darg} x(t)}{d t} \\
\psi^{\prime}(f)=-\frac{1}{2 \pi} \frac{\operatorname{darg} X(f)}{d f}
\end{gathered}
$$

donde $\varphi^{\prime}(t)$ es la frecuencia instantánea y $\psi^{\prime}(\omega)$ es el retardo de grupo. Como descripción general, se puede decir que la frecuencia instantánea nos da idea del valor frecuencial imperante en un determinado momento y que, a su vez, puede ser suma de varias componentes frecuenciales. Así, decimos que una representación tiempo-frecuencia cumple con la propiedad de la frecuencia instantánea si:

$$
\varphi^{\prime}(t)=\frac{\int f P(t, f) d f}{\int P(t, f) d f}
$$

Es decir, la frecuencia media de la representación tiempo-frecuencia, $P(t, f)$, en un determinado momento coincide con la frecuencia instantánea de la señal en ese instante. Por tanto, esta magnitud proporciona información útil solo si la señal contiene en cada instante un rango 
de frecuencias estrecho.

De forma análoga a la frecuencia instantánea, pero ahora integrando en función del tiempo, se define el retardo de grupo, que nos da la idea de los cambios de fase de las componentes de la señal con respecto a la portadora o, dicho de otra forma, del tiempo medio de llegada de una determinada frecuencia dentro de la señal analizada. Se dice que una representación tiempo-frecuencia cumple la propiedad del retardo de grupo si:

$$
\psi(f)=\frac{\int t P(t, f) d t}{\int P(t, f) d t}
$$

con lo que la media en la dirección temporal debe ser igual al retardo de grupo.

\subsection{Primera clase de solución: las descomposiciones atómicas}

Como es sabido, la transformada de Fourier no está adaptada para el análisis de señales no estacionarias, ya que proyecta la señal en sinusoides para los cuales no se puede determinar el momento en el que ocurren cuando la señal se observa en el dominio del tiempo. Los conceptos de frecuencia instantánea y retardo de grupo también son inherentemente no adaptados a un gran número de señales no estacionarias, aquellas que contienen más de un componente elemental, y en particular las señales de ruido. Por ende las soluciones mono-dimensionales no parecen ser suficientes, y se tienen que considerar funciones bidimensionales (funciones de las variables tiempo y frecuencia) [93].

Una primera clase de tales representaciones tiempo-frecuencia está dada por las descomposiciones atómicas (también conocidas como representaciones tiempo-frecuencia lineales). Para introducir este concepto, se comenzará con la transformada de Fourier de tiempo corto, que tiene una interpretación muy intuitiva.

UG - UVa

Paulo Antonio Delgado Arredondo 


\subsubsection{La transformada de Fourier en tiempo corto}

A fin de introducir tiempo-dependencia en la transformada de Fourier, una sencilla e intuitiva solución consiste en un pre-ventaneo de la señal $x(t)$ alrededor de un determinado tiempo $u$, calculando su transformada de Fourier, y repitiendo dicha operación para cada instante de tiempo $u$. La transformada resultante, llamada transformada de Fourier en tiempo corto (STFT, o espectro de tiempo corto), es [92]:

$$
F_{x}(u, f ; h)=\int_{-\infty}^{+\infty} x(t) h^{*}(t-u) e^{-j 2 \pi f t} d t
$$

donde $h(t)$ es una ventana de análisis de tiempo corto localizada alrededor de $t=0$ y $f=0$. Debido a que la multiplicación por la ventana relativamente corta $h^{*}(t-u)$ efectivamente suprime la señal fuera de la vecindad alrededor del punto de análisis $u=t$, la STFT es un espectro "local" de la señal x(t) alrededor de $u$. Siempre que la ventana de corta duración sea de energía finita, la STFT es invertible de acuerdo con:

$$
x(t)=\frac{1}{E_{h}} \int_{-\infty}^{+\infty} \int_{-\infty}^{+\infty} F_{x}(u, f ; h) h(t-u) e^{j 2 \pi t f} d u d f
$$

con $E_{h}=\int_{-\infty}^{+\infty}|h(t)|^{2} d t$. Esta relación expresa que la señal total puede ser descompuesta como una suma ponderada de formas de onda elementales

$$
h_{t, f}(t)=h(t-u) e^{j 2 \pi f t}
$$

las cuales pueden ser interpretadas como "bloques de construcción"ó "átomos". Cada átomo es obtenido de la ventana $h(t)$ por una traslación en tiempo y una traslación en frecuencia (modulación). La STFT puede también expresarse en términos del espectro de la señal y el espectro de la ventana.

$$
F_{x}(u, f ; h)=\int_{-\infty}^{+\infty} X(f) H^{*}\left(f-f_{0}\right) e^{j 2 \pi\left(f-f_{0}\right) t} d f
$$

donde $X(f)$ y $H(f)$ son las transformadas de Fourier de $x$ y $h$ respectivamente. Por lo tanto la STFT, $F_{x}(u, f ; h)$, puede ser considerada como el resultado de pasar la señal $x(t)$ a través de un filtro pasa-banda, es decir, la STFT es similar a un banco de filtros pasa-banda con ancho de banda constante. 


\subsubsection{Análisis tiempo-escala y la transformada wavelet}

La idea de la transformada wavelet continua (CWT) es proyectar una señal $x$ en una familia de funciones de media cero (wavelets) deducidas a partir de la función primaria (la wavelet madre) por traslaciones y dilataciones [92]:

$$
T_{x}(t, a ; \Psi)=\int_{-\infty}^{+\infty} x(s) \Psi_{t, a}^{*}(s) d s
$$

donde $\Psi_{t, a}(s)=|a|^{-1 / 2} \Psi\left(\frac{s-t}{a}\right)$. La variable $a$ corresponde ahora a un factor de escala, en el sentido de que tomando $|a|>1$ dilata la wavelet $\Psi$ y tomando $|a|<1$ la comprime. Por definición, la transformada wavelet es más una representación tiempo-escala que una representación tiempo-frecuencia. Sin embargo, para wavelets las cuales son bien localizadas alrededor de una frecuencia distinta de cero, $\nu_{0}$, en la escala $a=1$, una interpretación tiempo-frecuencia es posible gracias a la identificación formal $\nu=\frac{\nu_{0}}{a}$.

La diferencia básica entre la transformada Wavelet y la transformada de Fourier en tiempo corto es la siguiente: cuando el factor de escala $a$ es cambiado, la duración y el ancho de banda de la wavelet cambian pero su forma continua siendo la misma. Y en contraste con la STFT, la cual usa una sola ventana de análisis, la CWT usa ventanas pequeñas a altas frecuencias y ventanas grandes a bajas frecuencias, como se muestra en la Figura 3.1 . Esto supera parcialmente la limitación de resolución de la STFT: el ancho de banda $B$ es proporcional a $\nu$,ó

$$
\frac{B}{\nu}=Q
$$

donde $Q$ se conoce como constante de análisis. La CWT también puede ser vista como un banco de filtros de análisis, compuesto por filtros pasa-banda con ancho de banda relativamente constante. 


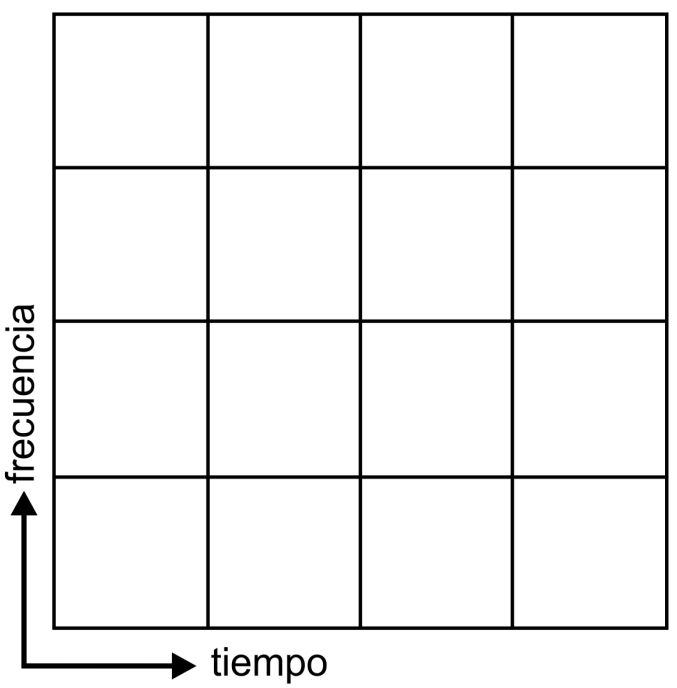

a)

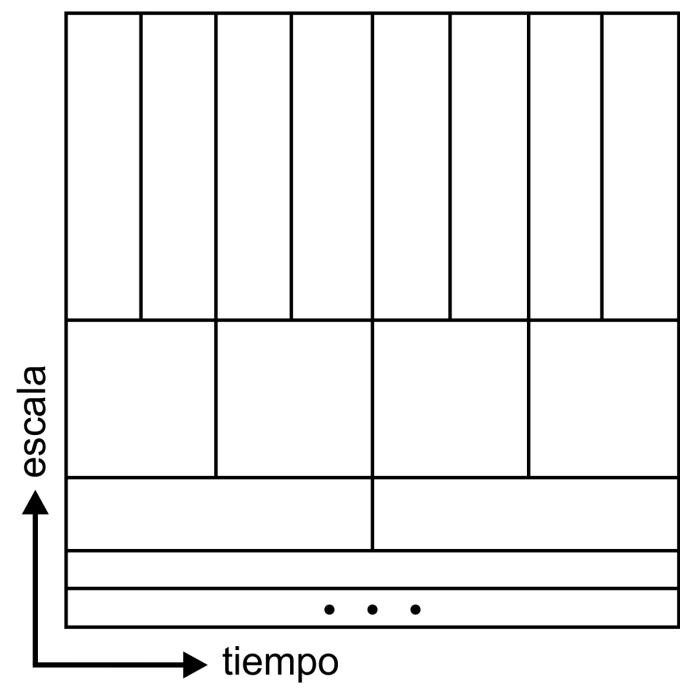

b)

Figura 3.1: Representación simbólica de las ventanas tiempo-frecuencia para la a)STFT y la b) CWT.

\subsubsection{De las descomposiciones atómicas a las distribuciones de energía}

Hasta este punto, se han presentado las representaciones tiempo-frecuencia que descomponen la señal en componentes elementales, los átomos, bien localizados en el tiempo y en frecuencia. Estas representaciones son transformadas lineales de la señal.

Otra solución al problema de caracterizar el contenido frecuencial de señales no estacionarias, que se desarrollará en la siguiente sección, consiste en la distribución de la energía de la señal a lo largo de las dos variables tiempo y frecuencia. Esto da lugar a las distribuciones de energía tiempo-frecuencia, que son transformadas naturalmente cuadráticas de la señal. Presentamos en esta sección una transición natural entre estas dos clases de soluciones a través del espectrograma (para el grupo de Weyl-Heisenberg) y el escalograma (para el grupo afín). 


\section{Espectrograma}

Si tenemos en cuenta el módulo al cuadrado de la STFT, se obtiene una densidad de energía conjunta, tanto en el dominio de la frecuencia como en el dominio temporal, de la señal ventaneada localmente $x(t) h^{*}(t-u)$ :

$$
S_{x}(u, f)=\left|\int_{-\infty}^{+\infty} x(t) h^{*}(t-u) e^{-j 2 \pi f t} d t\right|^{2}
$$

Esto define el espectrograma, que es una distribución de valores reales y no negativos. Dado que la ventana $h$ de la STFT es asumida de energía unitaria, el espectrograma satisface la propiedad de distribución de energía total:

$$
\int_{-\infty}^{+\infty} \int_{-\infty}^{+\infty} S_{x}(t, f) d t d f=E_{x}
$$

Así, podemos interpretar el espectrograma como una medida de la densidad de energía de la señal, contenida en el dominio tiempo-frecuencia centrada en el punto $(t, f)$ y cuya forma es independiente de esta localización 92 .

\section{Propiedades}

- Invarianza temporal y frecuencial

Una consecuencia directa de la definición del espectrograma es que conserva cambios de tiempo y de frecuencia:

$$
\begin{gathered}
y(t)=x\left(t-t_{0}\right) \Rightarrow S_{y}(t, f)=S_{x}\left(t-t_{0}, f\right) \\
y(t)=x(t) e^{j 2 \pi f_{0} t} \Rightarrow S_{y}(t, f)=S_{x}\left(t, f-f_{0}\right)
\end{gathered}
$$

Así, el espectrograma es un elemento de la clase de las distribuciones tiempo-frecuencia cuadráticas que son invariantes por la traslación en el tiempo y en la frecuencia. Esta clase, desarrollada en la sección siguiente, es llamada la clase de Cohen [94 [95]. 
- Resolución tiempo-frecuencia

El espectrograma es la magnitud al cuadrado de la STFT, es obvio que la resolución tiempo-frecuencia del espectrograma se limita exactamente de la misma forma que para la STFT. En particular, existe de nuevo un equilibrio entre la resolución de tiempo y resolución de frecuencia. Este establecimiento de una pobre resolución es el principal inconveniente de esta representación.

- Estructura de la interferencia

Como se trata de una representación cuadrática (o bilineal), el espectrograma de la suma de dos señales no es la suma de los dos espectrogramas (principio de superposición de segundo grado):

$$
y(t)=x_{1}(t)+x_{2}(t) \Rightarrow S_{y}(t, f)=S_{x_{1}}(t, f)+S_{x_{2}}(t, f)+2 \Re\left\{S_{x_{1}, x_{2}}(t, f)\right\}
$$

donde $S_{x_{1}, x_{2}}(t, f)=F_{x_{1}}(t, f) F_{x_{2}}^{*}(t, f)$ es el espectrograma cruzado y $\Re$ denota la parte real. Por lo tanto, como todas las distribuciones cuadráticas, el espectrograma presenta términos de interferencia, dados por $S_{x_{1}, x_{2}}(t, f)$. Sin embargo, se puede demostrar que estos términos de interferencia están restringidos a las regiones del plano tiempo-frecuencia donde los autoespectrogramas $S_{x_{1}}(t, f)$ y $S_{x_{2}}(t, f)$ se superponen. Así, si los componentes de la señal $x_{1}(t)$ y $x_{2}(t)$ son suficiente distantes para que sus espectrogramas no se solapen de manera significativa, entonces el término de interferencia casi será igual a cero. Esta propiedad, que es una ventaja práctica del espectrograma, es en realidad una consecuencia de la pobre resolución del mismo.

\section{Escalograma}

Una distribución similar al espectrograma se puede definir en el caso de la wavelet. Dado que la CWT se comporta como una descomposición en bases ortonormales, se puede 
demostrar que conserva la energía 93]:

$$
\int_{-\infty}^{+\infty} \int_{-\infty}^{+\infty}\left|T_{x}(t, a ; \Psi)\right|^{2} d t \frac{d a}{a^{2}}=E_{x}
$$

donde $E_{x}$ es la energía de $x$. Esto nos lleva a definir el escalograma de $x$ como el cuadrado del módulo de la CWT. Esta es una distribución de energía de la señal en el plano tiempo-escala, asociado con la medida del factor de escala $a$ en $d t \frac{d a}{a^{2}}$.

En cuanto a la transformada wavelet, las resoluciones en tiempo y en frecuencia del escalograma están relacionadas a través del principio de Heisenberg-Gabor: resoluciones en tiempo y frecuencia dependen de la frecuencia considerada. Una vez más, nos damos cuenta de que la resolución de la frecuencia es claramente una función de la frecuencia: esta se incrementa con $f$.

Los términos de interferencia del escalograma, como para el espectrograma, son también limitados a las regiones del plano tiempo-frecuencia donde los correspondientes autoescalogramas (términos de la señal) se superponen. Por lo tanto, si dos componentes de la señal están lo suficientemente separadas en el plano tiempo-frecuencia, su escalogramacruzado será esencialmente cero.

\subsection{Segunda clase de solución: distribuciones de energía}

En contraste con las representaciones lineales tiempo-frecuencia que descomponen la señal en componentes elementales (los átomos), el propósito de las distribuciones de energía es la de distribuir la energía de la señal en las dos variables de descripción: tiempo y frecuencia.

El punto de partida es que puesto que la energía de una señal $x$ se puede deducir del módulo al cuadrado tanto de la señal como de su transformada de Fourier,

$$
E_{x}=\int_{-\infty}^{+\infty}|x(t)|^{2} d t=\int_{-\infty}^{+\infty}|X(f)|^{2} d f
$$


podemos interpretar $|x(t)|^{2}$ y $|X(f)|^{2}$ como densidades de energía, respectivamente en el tiempo y en la frecuencia. Es entonces natural ver conjuntamente la densidad de energía en tiempo y frecuencia, $\rho_{x}(t, f)$, de tal manera que

$$
E_{x}=\int_{-\infty}^{+\infty} \int_{-\infty}^{+\infty} \rho_{x}(t, f) d t d f
$$

es una situación intermedia entre las descritas por la ecuación 3.25. Como la energía es una función cuadrática de la señal, las distribuciones de energía tiempo-frecuencia serán, en general, las representaciones cuadráticas.

Otras dos propiedades que la densidad de energía debe satisfacer son las siguientes propiedades marginales:

$$
\begin{gathered}
\int_{-\infty}^{+\infty} \rho_{x}(t, f) d t=|X(f)|^{2} \\
\int_{-\infty}^{+\infty} \rho_{x}(t, f) d f=|x(t)|^{2}
\end{gathered}
$$

lo que significa que si integramos la densidad de energía tiempo-frecuencia a lo largo de una variable, se obtiene la densidad de energía correspondiente a la otra variable.

\subsubsection{Propiedades de las representaciones tiempo-frecuencia}

Las representaciones tiempo-frecuencia muestran la evolución de las componentes frecuenciales de una señal a lo largo del tiempo. Pero en muchas ocasiones, para el caso de las representaciones cuadráticas, se desea que la representación tiempo-frecuencia nos muestre la densidad de energía de la señal que existe en cada punto del plano tiempo-frecuencia, con lo que la distribución se podría tratar como otro tipo de densidad de energía, pudiendo calcular momentos locales y globales.

Comprobando el cumplimiento de diversas propiedades, podremos averiguar qué condiciones deben cumplirse para que una representación tiempo-frecuencia corresponda a una densidad de energía en el sentido estricto de la palabra.

UG - UVa

Paulo Antonio Delgado Arredondo 


\section{Marginales}

Si se suman todos los términos de la distribución correspondientes a un mismo tiempo o frecuencia, debemos obtener el espectro de densidad de energía y la energía instantánea, respectivamente. Por tanto, las condiciones marginales de tiempo y frecuencia establecen:

$$
\begin{gathered}
P(f)=\int P(t, f) d t=|X(f)|^{2} \\
P(t)=\int P(t, f) d f=|x(t)|^{2}
\end{gathered}
$$

donde $P(t, f)$ corresponde con la densidad en el punto $(t, f)$ del plano tiempo-frecuencia. $|x(t)|^{2}$ es la intensidad de la señal en el tiempo $t$, y $|X(f)|^{2}$ la intensidad en la frecuencia $f$.

\section{Energía total}

Si la transformada tiempo-frecuencia corresponde a una densidad de energía, se debe cumplir que:

$$
\iint P(t, f) d f d t=\int|x(t)|^{2} d t=\int|X(f)|^{2} d f
$$

Esta propiedad se cumple automáticamente si los marginales lo hacen, aunque lo contrario no es cierto.

\section{Invarianza ante desplazamientos temporales y frecuenciales}

Sea una señal $x(t)$, si tiene desplazamiento en el tiempo:

$$
\tilde{x}(t)=x\left(t-t_{0}\right)
$$

la representación tiempo-frecuencia correspondiente a dicha señal es invariante ante desplazamientos temporales cuando se cumple:

$$
P_{\tilde{x}}(t, f)=P_{x}\left(t-t_{0}, f\right)
$$

Si en cambio tenemos que para la señal $x(t)$ existe un desplazamiento en frecuencia:

$$
\tilde{x}(t)=x(t) e^{j 2 \pi f_{0} t}
$$


la representación tiempo-frecuencia cumple la propiedad de invarianza ante desplazamientos frecuenciales si:

$$
P_{\tilde{x}}(t, f)=P_{x}\left(t, f-f_{0}\right)
$$

\section{Escalado lineal}

Dada una señal $x(t)$, para una constante $a$, la version escalada de la señal es $x_{c}(t)=$ $\sqrt{a} x(a t)$, con lo que $x_{c}(t)$ es expandida o reducida dependiendo de si $a$ es mayor o menor que la unidad. Obteniendo el espectro de la señal:

$$
X_{c}(f)=\frac{1}{\sqrt{a}} X\left(\frac{f}{a}\right)
$$

Como se aprecia, si la señal se comprime, el espectro se expande y viceversa. Para que esta propiedad se cumpla dentro del ámbito de las distribuciones tiempo-frecuencia, debemos obtener que:

$$
P_{x_{c}}(t, f)=P_{x}\left(a t, \frac{f}{a}\right)
$$

\subsubsection{La clase de Cohen}

En 1966, Leon Cohen utilizó funciones características junto con la teoría de operadores para desarrollar una clase general de distribuciones tiempo-frecuencia bilineales. Este trabajo produjo un avance significativo en el estudio y desarrollo de las representaciones tiempofrecuencia, ya que el problema de diseñar estas representaciones se reduce al hecho de elegir una función, llamada kernel, en la que siguiendo ciertas normas se puede asegurar el buen comportamiento de la representación elegida. Esto permite seleccionar la función kernel que se considere apropiada para que se cumplan determinadas propiedades, y que genere una distribución tiempo-frecuencia de acuerdo con nuestras necesidades.

Como definición, diremos que una representación tiempo-frecuencia pertenece a la clase de Cohen si satisface las propiedades de invarianza ante desplazamientos temporales e invarianza 
ante desplazamientos frecuenciales. De esta forma, cualquier distribución tiempo-frecuencia se puede obtener mediante la expresión [94]:

$$
\operatorname{TFR}(t, \omega)=\iint A F(\tau, v) \Phi(\tau, v) e^{j(t v-\omega \tau)} d \tau d v
$$

En la ecuación (3.38), $\Phi(\tau, v)$ constituye el kernel y $A F(\tau, v)$ es la función de ambigüedad correspondiente a la señal origen de la representación que se define como:

$$
A F(\tau, v)=\frac{1}{2 \pi} \int_{-\infty}^{+\infty} x\left(t+\frac{\tau}{2}\right) x^{*}\left(t-\frac{\tau}{2}\right) e^{-j 2 \pi v t} d t
$$

Además, cualquier representación tiempo-frecuencia de esta clase puede considerarse como una variante de la representación Wigner-Ville a la que se añade un kernel, que generalmente corresponde a un filtrado de la representación tiempo-frecuencia de Wigner-Ville, por lo que a veces reciben el nombre de representaciones suavizadas de Wigner-Ville, aunque debido a la definición de características propias por parte de algunas de ellas actualmente reciben un nombre especifico. La representación de Wigner-Ville está definida por:

$$
W V=(t, \omega)=\int x\left(t+\frac{\tau}{2}\right) x^{*}\left(t-\frac{\tau}{2}\right) e^{-j \omega \tau} d \tau
$$

En función de la ecuación 3.40 se puede obtener una representación general de la Clase de Cohen, que se muestra en la ecuación (3.41).

$$
T F R(t, \omega)=\iint \Phi(t-u, \omega-\nu) W V(u, \nu) d u d \nu
$$

La elección del núcleo o kernel es de gran importancia, ya que de este depende que se cumplan determinadas propiedades. A menudo, estos núcleos se pueden interpretar como un filtro bidimensional cuya principal función es la de proporcionar una buena eliminación de los términos cruzados sin provocar alta distorsión de los términos propios de la señal.

Dado que se manipulan funciones cuadráticas con respecto a la señal original, toma gran importancia la función autocorrelación, ya que dependiendo de cómo se defina, dará lugar a 
diferentes tipos de representaciones. La densidad de energía espectral obtenida a partir de la función de autocorrelación viene dada por:

$$
\begin{gathered}
|S(\omega)|^{2}=\int R(\tau) e^{-j \omega \tau} d \tau \\
R(\tau)=\int s^{*}(t) s(t+\tau) d t=\int s^{*}(t-\tau) s(t) d t=\int s^{*}\left(t-\frac{\tau}{2}\right) s\left(t+\frac{\tau}{2}\right) d t
\end{gathered}
$$

Generalizando el concepto de autocorrelación, una representación tiempo-frecuencia se puede expresar como:

$$
P(t, \omega)=\int R_{t}(\tau) e^{-j \omega \tau} d \tau
$$

En este caso $R_{t}(\tau)$ es una función de autocorrelación local variable en el tiempo.

\subsubsection{La clase afín}

Se dice que una distribución tiempo-frecuencia pertenece a la clase afín cuando cumple la propiedad de ser invariante ante cambios de escala y ante traslaciones temporales. La propiedad de ser invariante ante cambios de escala resulta bastante útil en señales con transitorios de corta duración, pues ello permite una mejor apreciación dependiendo de la escala que se emplee.

Análogamente a las representaciones de la clase de Cohen, en la ecuación (3.45) se expresa de manera general una distribución perteneciente a la clase afín:

$$
T F R(t, f)=\iint A F(\tau, \nu) \Phi\left(f \tau, \frac{\nu}{f}\right) e^{j 2 \pi \nu t} d \tau d \nu
$$

En la ecuación anterior, $\Phi\left(f \tau, \frac{\nu}{f}\right)$ constituye el kernel y $A F(\tau, \nu)$ es la función de ambigüedad correspondiente a la señal origen de la representación.

Al igual que ocurre en el caso de la clase de Cohen, estas distribuciones se pueden clasificar dependiendo de las propiedades que satisfacen.

UG - UVa

Paulo Antonio Delgado Arredondo 


\subsection{Distribución tiempo-frecuencia de Gabor}

Un caso particular de la transformada de Fourier en tiempo corto es la distribución tiempo-frecuencia de Gabor. Esta distribución tiempo-frecuencia utiliza una ventana del tipo Gaussiana y la transformada de Fourier para obtener una representación tiempo-frecuencia [96]. Al igual que sucede con la STFT, en la distribución tiempo-frecuencia de Gabor (TFDG) existe el compromiso de resolución entre tiempo y frecuencia, causado por la longitud de la ventana. Sin embargo, en la TFDG, debido a las características de la ventana Gaussiana, relativamente se obtiene una mejor representación tiempo-frecuencia a causa del suavizado generado por la ventana. La TFDG en su forma continua se define por la siguiente ecuación:

$$
G_{x}(t, f)=\int_{-\infty}^{\infty} e^{-\pi(\tau-t)^{2}} e^{-j 2 \pi f \tau} x(\tau) d \tau
$$

Como sucede en el caso continuo, en el caso discreto, la TFDG es idéntica a la STFT discreta, con la diferencia particular de la ventana Gaussiana. La señal de analisis discreta se puede expandir como una combinación lineal de coeficientes, llamados coeficientes de Gabor y funciones básicas [97, 98. Para un conjunto finito de datos x(n), la expansión de la señal en coeficientes de Gabor es la siguiente:

$$
x(n)=\sum_{m=0}^{M} \sum_{k=0}^{N} a_{m, k} g(n-m N) e^{j 2 \pi k n / K}
$$

donde el arreglo $a_{m, k}$ es periódico en $\mathrm{k}$ y con periodo K. La secuencia $g(n)$ es la ventana de síntesis. La matriz de los coeficientes de Gabor, $a_{m, k}$ se puede encontrar a través de la TFDG:

$$
a_{m, k}=\sum_{n=0}^{N-1} x(n) w^{*}(n-m N) e^{-j 2 \pi k n / K}
$$

donde la secuencia $w(n)$ es la ventana de análisis. La transformada Zak se utiliza para obtener los coeficientes de Gabor. La transformada Zak discreta de una ventana periódica se define como la transformada discreta de Fourier mono-dimensional de la secuencia $W(n-m N)$ :

$$
Z=\sum_{m=0}^{N-1} W(n-m N) e^{-j 2 \pi m n(l / M N)}
$$

donde $n$ y $l$ son parámetros de ajuste, $M$ es el periodo y $N$ es el número de muestras.

UG - UVa

Paulo Antonio Delgado Arredondo 


\subsection{Escalograma de Morlet}

Como se mencionó anteriormente, dado que la CWT se comporta como una descomposición de bases ortonormales, se puede demostrar que conserva la energía. Esta característica es la que lleva a la definición del escalograma, que es el cuadrado del módulo de la CWT.

Así mismo, la idea de la CWT es la de proyectar una señal $x$ en una familia de funciones de media cero (wavelets) deducidas a partir de la función primaria (la wavelet madre). Una de las wavelets madre más usadas es la Morlet que se define como:

$$
\psi(t)=\frac{1}{\sqrt[4]{\pi}}\left(e^{j \omega_{0} t}-e^{-\omega_{0}^{2} / 2}\right) e^{-t^{2} / 2 \sigma^{2}}
$$

donde $\omega_{0}$ es la frecuencia central de la wavelet madre. El termino $e^{-\omega_{0} / 2}$ se usa para corregir la media diferente de cero de las sinusoides complejas. Este término puede ser omitido para valores de $w_{0}>5$. Por lo tanto, en algunos trabajos de investigación la definición de la wavelet madre de Morlet está dada por:

$$
\psi(t)=\frac{1}{\sqrt[4]{\pi}} e^{j \omega_{0} t} e^{-t^{2} / 2 \sigma^{2}}
$$

donde la frecuencia central cumple que $\omega_{0}>5$. El escalograma de Morlet (TFMS) tiene una forma muy similar a la transformada de Gabor. La diferencia importante es que la función ventana esta escalada en lugar de ser fija [99].

\subsection{Extracción de información de una imagen tiempo- frecuencia}

Hasta este punto, se han examinado soluciones propuestas al problema de representar una señal no estacionaria en el plano tiempo-frecuencia. Ahora se tiene que considerar el problema de la interpretación de la imagen tiempo-frecuencia que describe la evolución con el tiempo del contenido de frecuencia de la señal. Incluso si todas las representaciones tiempo-frecuencia tienen el mismo objetivo, cada representación tiene que ser interpretada de manera diferente, 
de acuerdo con sus propiedades características. Por ejemplo, algunas de las representaciones presentan considerables términos de interferencia, en otras los términos de interferencia son despreciables, en algunas señales particulares los términos son perfectamente localizados, etcétera. Así la extracción de información tiene que hacerse con cuidado, partiendo del conocimiento de las propiedades de las representaciones tiempo-frecuencia que se utilicen.

Los momentos y marginales de algunas representaciones proporcionan importante información acerca de la señal, como su modulación de amplitud o su frecuencia instantánea, por ejemplo, con el objetivo de demodular la señal.

\subsubsection{Momentos}

Los momentos de primero y segundo orden en tiempo de una distribución de energía tiempo-frecuencia $(t f r)$ se definen como:

$$
\begin{gathered}
f_{m}(t)=\frac{\int_{-\infty}^{+\infty} f t f r(t, f) d f}{\int_{-\infty}^{+\infty} t f r(t, f) d f} \\
B^{2}(t)=\frac{\int_{-\infty}^{+\infty} f^{2} t f r(t, f) d f}{\int_{-\infty}^{+\infty} t f r(t, f) d f}-f_{m}(t)^{2}
\end{gathered}
$$

y para los momentos de primero y segundo orden en frecuencia de una distribución tiempofrecuencia como:

$$
\begin{gathered}
t_{m}(f)=\frac{\int_{-\infty}^{+\infty} t \operatorname{tfr}(t, f) d t}{\int_{-\infty}^{+\infty} t f r(t, f) d t} \\
T^{2}(f)=\frac{\int_{-\infty}^{+\infty} t^{2} t f r(t, f) d t}{\int_{-\infty}^{+\infty} t f r(t, f) d t}-t_{m}(f)^{2}
\end{gathered}
$$

Los momentos describen las posiciones promedio y se extiende en el tiempo y en la frecuencia de la señal. Para algunas distribuciones particulares, si la señal se considera en su forma analítica, el momento de primer orden en el tiempo corresponde a la frecuencia instantánea, y el momento de primer orden en la frecuencia al retardo de grupo de la señal. 


\subsubsection{Marginales}

También puede ser interesante tener en cuenta las distribuciones marginales de una representación tiempo-frecuencia. El marginal temporal se define como:

$$
m_{f}(t)=\int_{-\infty}^{+\infty} t f r(t, f) d f
$$

el marginal frecuencial se define como:

$$
m_{t}(f)=\int_{-\infty}^{+\infty} t f r(t, f) d t
$$

y expresan, mediante la integración de la representación a lo largo de una variable, el reparto de la energía a lo largo de la otra variable. Una limitación natural para una distribución tiempo-frecuencia es que el marginal en tiempo corresponde a la potencia instantánea de la señal, y que el marginal en frecuencia corresponde a la densidad espectral de energía:

$$
m_{f}(t)=|x(t)|^{2} y m_{t}(f)=|X(f)|^{2}
$$

\subsection{Estimación de la densidad espectral de potencia}

En esta sección se abordará el problema de la estimación de la densidad espectral de potencia. La densidad espectral de potencia es la transformada de Fourier de la secuencia de autocorrelación. Por lo tanto, el problema de estimar la densidad espectral de potencia se reduce a estimar la secuencia de autocorrelación de la señal analizada. El cálculo de la secuencia de autocorrelación de un proceso ergódico, se puede hacer a partir de la siguiente ecuación 100

$$
\lim _{N \longrightarrow \infty}\left\{\frac{1}{2 N+1} \sum_{n=-N}^{N} x(n+k) x^{*}(n)\right\}=r_{x}(k)
$$

donde $N$ es el número de muestras de la señal digital, $x(n)$ es la señal, $x^{*}(n)$ es su complejo conjugado y $r_{x}(k)$ es la secuencia de autocorrelación. Por lo tanto, si se conoce la señal $x(n)$ para todo valor de $n$, la estimación de la densidad espectral de potencia se hará de manera relativamente sencilla con la ayuda de la ecuación (3.59) y posteriormente obteniendo su 
transformada de Fourier. A pesar de esto, existen dos dificultades con esta aproximación que hacen que la estimación de la densidad espectral de potencia sea interesante y un reto. La primera dificultad es que la cantidad de datos con los que se cuenta siempre son limitados y en muchas ocasiones son mínimos. Esta limitación en la cantidad de datos disponibles se debe inherentemente al fenómeno que se esté analizando, por ejemplo, cuando se obtienen datos del transitorio de arranque de un motor, este puede durar solo unos pocos segundos y además de que su contenido espectral deberá permanecer constante, aunque sea en un instante de tiempo aún más corto que la duración del transitorio mismo. Por ejemplo, en el habla, que es una señal de sonido, el requisito de la estacionariedad restringe la duración de la señal a unos pocos milisegundos, haciendo que la cantidad de datos con los que se cuenta sea muy reducida. La segunda dificultad que se presenta para el cálculo de la densidad espectral de potencia es que las señales generalmente están contaminadas con ruido o presentan interferencias con otras señales. Por lo tanto la estimación de la densidad espectral de potencia es un problema que envuelve el cálculo de la potencia de exponenciales complejos $\left(P_{x}\left(e^{j w}\right)\right)$ de un numero finito de muestras $x(n)$ con ruido.

Los métodos que se han desarrollado para estimar la densidad espectral de potencia pueden categorizarse en dos clases. La primera de estas clases incluye los métodos clásicos o no paramétricos que se basan en la estimación de la secuencia de autocorrelación $r_{x}(k)$ a partir de los datos de la señal. Después de que se obtiene la secuencia de autocorrelación se calcula su transformada de Fourier para así obtener la densidad espectral de potencia. El segundo grupo incluye los métodos no clásicos o paramétricos, estos métodos se basan en un modelo matemático previamente definido para la estimación de la densidad espectral de potencia.

Los métodos que se consideran clásicos o no paramétricos incluyen el periodograma, el periodograma modificado, el método de Bartlett, el método de Welch y el método de BlackmanTuckey. Los métodos para estimar la densidad espectral de potencia que se basan en modelos paramétricos para los datos son: el método de promedio móvil (MA, acrónimo de Moving Average), el método autorregresivo (AR, acrónimo de Autorregresive), el método autorregresivo de promedio móvil (ARMA, acrónimo de Autorregresive Moving Average) y los métodos 
de estimación que consisten en la descomposición harmónica de sinusoides o exponenciales complejos inmersos en ruido. Estos métodos de descomposición armónica algunas veces son llamados métodos de subespacio del ruido; entre estos métodos se encuentra la descomposición harmónica de Pisarenko y MUSIC (acrónimo de Multiple Signal Classification).

\subsubsection{Descomposición armónica}

El modelo utilizado para la descomposición armónica, es un modelo en el cual la señal $x(n)$ es una suma de exponenciales complejos en ruido blanco, como se define en la siguiente ecuación.

$$
x(n)=\sum_{i=1}^{p} A_{i} e^{j n \omega_{i}}+w(n)
$$

En esta ecuación se asume que las amplitudes $A_{i}$ son complejas,

$$
A_{i}=\left|A_{i}\right| e^{j \phi_{i}}
$$

donde $\phi_{i}$ son variables aleatorias no correlacionadas que están uniformemente distribuidas en el intervalo $[-\pi, \pi]$. Ademas a pesar de que las frecuencias y magnitudes de los exponenciales complejos, $\omega_{i}$ y $\left|A_{i}\right|$, respectivamente, no son aleatorias, se asume que son desconocidas. Por lo tanto, la densidad espectral de potencia de $x(n)$ consiste de un conjunto $p$ de impulsos de área $2 \pi\left|A_{i}\right|$, a una frecuencia $\omega_{i}$ para $i=1,2, \ldots, p$, más la densidad espectral de potencia del ruido aditivo $w(n)$. Los métodos que estiman la densidad espectral de potencia utilizando este modelo se basan en la eigen-descomposición de la matriz de autocorrelación en dos subespacios, el subespacio de la señal y el subespacio del ruido. Una vez que estos subespacios son determinados, entonces una función de estimación de frecuencia se usa para extraer las frecuencias.

Para explicar la eigen-descomposición de la matriz de autocorrelación, comenzaremos con un proceso de primer orden:

$$
x(n)=A_{1} e^{j n \omega_{1}}+w(n)
$$

Este proceso consiste de un exponencial complejo en ruido blanco. La amplitud del exponencial complejo es $A_{1}=\left|A_{1}\right| e^{j \phi_{1}}$, donde $\phi_{1}$ es una variable aleatoria uniformemente distribuida 
y $w(n)$ es ruido blanco que tiene varianza igual a $\sigma_{w}^{2}$. Para este proceso la secuencia de autocorrelación de $x(n)$ es,

$$
r_{x}(k)=P_{1} e^{j k \omega_{1}}+\sigma_{w}^{2} \delta(k)
$$

donde $P_{1}=\left|A_{1}\right|^{2}$ es la potencia en el exponencial complejo. Por lo tanto, la matriz de autocorrelacion $M x M$ para $x(n)$ es una suma de una matriz de autocorrelación debido a la señal $\mathbf{R}_{s}$, y una matriz de autocorrelación debido al ruido $\mathbf{R}_{n}$

$$
\mathbf{R}_{x}=\mathbf{R}_{s}+\mathbf{R}_{n}
$$

donde la matriz de autocorrelacion de la señal es igual a

$$
\mathbf{R}_{s}=P_{1}\left[\begin{array}{ccccc}
1 & e^{-j \omega_{1}} & e^{-j 2 \omega_{1}} & \ldots & e^{-j(M-1) \omega_{1}} \\
e^{j \omega_{1}} & 1 & e^{-j \omega_{1}} & \ldots & e^{-j(M-2) \omega_{1}} \\
e^{j 2 \omega_{1}} & e^{j \omega_{1}} & 1 & \ldots & e^{-j(M-3) \omega_{1}} \\
\vdots & \vdots & \vdots & & \vdots \\
e^{j(M-1) \omega_{1}} & e^{j(M-2) \omega_{1}} & e^{j(M-3) \omega_{1}} & \ldots & 1
\end{array}\right]
$$

la cual tiene rango uno, y la matriz de autocorrelación del ruido es diagonal,

$$
\mathbf{R}_{n}=\sigma_{w}^{2} \mathbf{I}
$$

y tiene rango completo. Ahora si definimos

$$
\mathbf{e}_{1}=\left[1, e^{j \omega_{1}}, e^{j \omega_{2}}, \ldots, e^{j \omega_{(M-1)}}\right]^{T}
$$

entonces $\mathbf{R}_{s}$ puede ser escrito en términos de $\mathbf{e}_{1}$ como sigue:

$$
\mathbf{R}_{s}=P_{1} \mathbf{e}_{1} \mathbf{e}_{1}^{H}
$$

ya que el rango de $\mathbf{R}_{s}$ es igual a uno, entonces $\mathbf{R}_{s}$ solo tiene un eigenvalor diferente de cero. Con

$$
\mathbf{R}_{s} \mathbf{e}_{1}=P_{1}\left(\mathbf{e}_{1} \mathbf{e}_{1}^{H}\right) \mathbf{e}_{1}=P_{1} \mathbf{e}_{1}\left(\mathbf{e}_{1}^{H}\right) \mathbf{e}_{1}=M P_{1} \mathbf{e}_{1}
$$

resulta que el eigenvalor diferente de cero es igual a $M P_{1}$, y que $\mathbf{e}_{1}$ es el eigenvector correspondiente. Además, ya que $\mathbf{R}_{s}$ es Hermitiana entonces los vectores restantes, $\mathbf{v}_{2}, \mathbf{v}_{3}, \ldots, \mathbf{v}_{M}$ serán ortogonales a $\mathbf{e}_{1}$,

$$
\mathbf{e}_{1}^{H} \mathbf{v}_{i}=0 ; \quad i=2,3, \ldots, M
$$


Finalmente, teniendo en cuenta que si permitimos que $\lambda_{i}^{s}$ sean los eigenvalores de $\mathbf{R}_{s}$, entonces:

$$
\mathbf{R}_{x} \mathbf{v}_{i}=\left(\mathbf{R}_{s}+\sigma_{w}^{2} \mathbf{I}\right) \mathbf{v}_{i}=\lambda_{i}^{s} \mathbf{v}_{i}+\sigma_{w}^{2} \mathbf{v}_{i}=\left(\lambda_{i}^{s}+\sigma_{w}^{2}\right) \mathbf{v}_{i}
$$

Por lo tanto, los eigenvectores de $\mathbf{R}_{x}$ son los mismos que aquellos para $\mathbf{R}_{s}$, y los eigenvalores de $\mathbf{R}_{x}$ son:

$$
\lambda_{i}=\lambda_{i}^{s}+\sigma_{w}^{2}
$$

Como resultado, el eigenvalor más grande de $\mathbf{R}_{x}$ es

$$
\lambda_{\max }=M P_{1}+\sigma_{w}^{2}
$$

y los $M-1$ eigenvalores restantes son iguales a $\sigma_{w}^{2}$. En consecuencia, es posible extraer todos los parámetros de interés acerca de $x(n)$ de los eigenvalores y eigenvectores de $\mathbf{R}_{x}$ como sigue:

1. Realizar una eigendescomposición de la matriz de autocorrelación $\mathbf{R}_{x}$. El eigenvalor más grande será igual a $M P_{1}+\sigma_{w}^{2}$ y los eigenvalores restantes serán iguales a $\sigma_{w}^{2}$.

2. Usar los eigenvalores de $\mathbf{R}_{x}$ para encontrar la potencia $P_{1}$ y la varianza del ruido como sigue:

$$
\begin{gathered}
\sigma_{w}^{2}=\lambda_{\min } \\
P_{1}=\frac{1}{M}\left(\lambda_{\max }-\lambda_{\min }\right)
\end{gathered}
$$

3. Determinar la frecuencia $\omega_{1}$ a partir del eigenvector $\mathbf{v}_{\max }$ que está asociado con el eigenvalor más grande usando, por ejemplo, el segundo coeficiente de $\mathbf{v}_{\max }$,

$$
\omega_{i}=\arg \left\{v_{\max }(1)\right\}
$$

En la práctica, esta aproximación descrita tiene un valor de estimación limitado en frecuencia ya que requiere conocer de manera exacta la matriz de autocorrelación. Se puede emplear una matriz de autocorrelación estimada en lugar de $\mathbf{R}_{x}$. Pero si se hace esto entonces el eigenvalor más grande solo será aproximadamente igual a $P_{1}+\sigma_{w}^{2}$ y su eigenvector correspondiente solo será una aproximación de $\mathbf{e}_{1}$. Ya que los eigenvalores y los eigenvectores pueden ser bastante sensibles a pequeños errores en $r_{x}(k)$, en lugar de estimar la frecuencia 
del exponencial complejo a partir de un solo eigenvector, se debe considerar usar un promedio ponderado, como se explica a continuación. Sea $\mathbf{v}_{i}$ un eigenvector de ruido de $\mathbf{R}_{x}$, es decir, uno que tiene un eigenvalor de $\sigma_{w}^{2}$, y $v_{i}(k)$ sea el k-ésimo componente de $\mathbf{v}_{i}$. Si se calcula la transformada discreta de Fourier de los coeficientes en $\mathbf{v}_{i}$,

$$
V_{i}\left(e^{j \omega}\right)=\sum_{k=0}^{M-1} v_{i}(k) e^{-j \omega k}=\mathbf{e}^{H} \mathbf{v}_{i}
$$

entonces la condición de ortogonalidad dada en la ecuación (3.70) implica que $V_{i}\left(e^{j \omega k}\right)$ será igual a cero en $\omega=\omega_{1}$, la frecuencia del exponencial complejo. Por lo tanto, si se construye la función de estimación de frecuencia:

$$
\hat{P}_{i}\left(e^{j \omega}\right)=\frac{1}{\left|\sum_{k=0}^{M-1} v_{i}(k) e^{-j k \omega}\right|^{2}}=\frac{1}{\left|\mathbf{e}^{H} \mathbf{v}_{i}\right|^{2}}
$$

entonces, $\hat{P}_{i}\left(e^{j \omega}\right)$ será mayor en $\omega=\omega_{1}$. En consecuencia, la localización del pico de esta función de estimación puede ser usado para estimar la frecuencia del exponencial complejo. Sin embargo, la ecuación (3.78) usa solamente un eigenvector y, por lo tanto puede ser sensible a errores en la estimación de $\mathbf{R}_{x}$. Para evitar esto, se puede utilizar un promedio ponderado de todos los eigenvectores de ruido como sigue:

$$
\hat{P}\left(e^{j \omega}\right)=\frac{1}{\sum_{i=2}^{M} \alpha_{i}\left|\mathbf{e}^{H} \mathbf{v}_{i}\right|^{2}}
$$

donde $\alpha_{i}$ son algunas constantes escogidas apropiadamente.

Ahora consideremos el caso de dos exponenciales complejos en ruido blanco,

$$
x(n)=A_{1} e^{j n \omega_{1}}+A_{2} e^{j n \omega_{2}}+w(n)
$$

donde $A_{i}=\left|A_{i}\right| e^{j n \phi_{i}}$ para $i=1,2$ son las amplitudes de los exponenciales complejos, y $\omega_{1} \mathrm{y}$ $\omega_{2}$ son las frecuencias con $\omega_{1} \neq \omega_{2}$. Si la varianza de $w(n)$ es $\sigma_{w}^{2}$ entonces la autocorrelación de $x(n)$ es:

$$
r_{x}(k)=P_{1} e^{j k \omega_{1}}+P_{2} e^{j k \omega_{2}}+\sigma_{w}^{2} \delta(k)
$$


donde $P_{1}=\left|A_{1}\right|^{2}$ y $P_{2}=\left|A_{2}\right|^{2}$ para $i=1,2$. Por lo tanto, la matriz de autocorrelación se puede escribir como la suma,

$$
\mathbf{R}_{x}=P_{1} \mathbf{e}_{1} \mathbf{e}_{1}^{H}+P_{2} \mathbf{e}_{2} \mathbf{e}_{2}^{H}+\sigma_{w}^{2} \mathbf{I}
$$

donde

$$
\mathbf{R}_{s}=P_{1} \mathbf{e}_{1} \mathbf{e}_{1}^{H}+P_{2} \mathbf{e}_{2} \mathbf{e}_{2}^{H}
$$

esta es una matriz de rango dos que representa el componente de $\mathbf{R}_{x}$ debido a la señal y

$$
\mathbf{R}_{n}=\sigma_{w}^{2} \mathbf{I}
$$

es una matriz diagonal que se debe al ruido. Otra forma de expresar esta descomposición es escribir $\mathbf{R}_{x}$ como sigue:

$$
\mathbf{R}_{x}=\mathbf{E P E}^{H}+\sigma_{w}^{2} \mathbf{I}
$$

donde

$$
\mathbf{E}=\left[\mathbf{e}_{1}, \mathbf{e}_{2}\right]
$$

es una matiz $M \times 2$ que contiene los dos vectores de la señal e1 y e2 y $\mathbf{P}=\operatorname{diag}\left\{P_{1}, P_{2}\right\}$ es una matriz diagonal que contiene la potencia de la señal. Ademas de la descomposición de $\mathbf{R}_{x}$ como la suma de dos matrices de autocorrelación como en la ecuación (3.85), también se puede realizar una eigendescomposición de $\mathbf{R}_{x}$ como sigue. Permita $\mathbf{v}_{i}$ y $\lambda_{i}$ ser vectores y eigenvalores de $\mathbf{R}_{x}$, respectivamente, con los eigenvalores ordenados de forma decreciente,

$$
\lambda_{1} \geq \lambda_{2} \geq \cdots \geq \lambda_{M}
$$

Ya que $\mathbf{R}_{x}=\mathbf{R}_{s}+\sigma_{w}^{2} \mathbf{I}$ entonces

$$
\lambda_{i}=\lambda_{i}^{s}+\sigma_{w}^{2}
$$

donde $\lambda_{i}^{s}$ son los eigenvalores de $\mathbf{R}_{s}$. Como el rango de $\mathbf{R}_{s}$ es igual a dos, entonces $\mathbf{R}_{s}$ tiene solo dos eigenvalores diferentes de cero, y ambos son mayores que cero. Por lo tanto, los primeros dos eigenvalores de $\mathbf{R}_{x}$ son mayores que $\sigma_{w}^{2}$ y los eigenvalores restantes son iguales a $\sigma_{w}^{2}$. Asi, los eigenvalores y eigenvectores de $\mathbf{R}_{x}$ se pueden dividir en dos grupos. El primer grupo, que consiste en los dos eigenvectores que tienen los eigenvalores mayores que $\sigma_{w}^{2}$, 
que se denominan eigenvectores de la señal y abarcan un subespacio bidimensional llamado subespacio de la señal. El segundo grupo, que consiste de aquellos eigenvectores que tienen eigenvalores igual a $\sigma_{w}^{2}$, se denominan eigenvectores de ruido y abarcan un subespacio de dimension ( $M-2)$ llamado subespacio de ruido. Ya que $\mathbf{R}_{x}$ es Hermitiana, los eigenvectores $\mathbf{v}_{i}$ forman un conjunto ortonormal. Por lo tanto, los subespacios de la señal y el ruido son ortogonales, es decir, para cualquier vector $\mathbf{u}$ en el subespacio de la señal y para cualquier vector $\mathbf{v}$ en el subespacio del ruido, $\mathbf{u}^{H} \mathbf{v}=0$.

A diferencia del caso para un solo exponencial complejo, con una suma de dos exponenciales complejos en ruido, los eigenvectores de la señal generalmente no serán iguales a $\mathbf{e}_{1}$ y $\mathbf{e}_{2}$. Sin embargo, $\mathbf{e}_{1}$ y $\mathbf{e}_{2}$ estarán contenidos en el subespacio de la señal que está contenido por los eigenvectores $\mathbf{v}_{1}$ y $\mathbf{v}_{2}$, y debido a que el espacio de la señal y el espacio del ruido son ortogonales, entonces $\mathbf{e}_{1}$ y $\mathbf{e}_{2}$ serán ortogonales a los eigenvectores del ruido $\mathbf{v}_{i}$, es decir

$$
\begin{aligned}
& \mathbf{e}_{1}^{H} \mathbf{v}_{i}=0 ; \quad i=3,4, \ldots, M \\
& \mathbf{e}_{2}^{H} \mathbf{v}_{i}=0 ; \quad i=3,4, \ldots, M
\end{aligned}
$$

Por lo tanto, como en el caso de un exponencial complejo, las frecuencias de los exponenciales complejos, $\omega_{1}$ y $\omega_{2}$, pueden ser estimados usando una función de estimación de frecuencia de la forma:

$$
\hat{P}\left(e^{j \omega}\right)=\frac{1}{\sum_{i=3}^{M} \alpha_{i}\left|\mathbf{e}^{H} \mathbf{v}_{i}\right|^{2}}
$$

Ahora consideremos el caso general de un proceso estacionario en sentido amplio que consiste de $p$ distintos exponenciales complejos en ruido blanco. La secuencia de autocorrelación $M \times M$ es,

$$
r_{x}(k)=\sum_{i=1}^{p} P_{i} e^{j k \omega_{i}}+\sigma_{w}^{2} \delta(k)
$$

donde $P_{i}=\left|A_{i}\right|^{2}$ es la potencia en el i-ésimo componente exponencial. Por consiguiente, la matriz de autocorrelación se puede escribir como

$$
\mathbf{R}_{x}=\mathbf{R}_{s}+\mathbf{R}_{n}=\sum_{i=1}^{p} P_{i} \mathbf{e}_{i} \mathbf{e}_{i}^{H}+\sigma_{w}^{2} \mathbf{I}
$$


donde

$$
\mathbf{e}_{i}=\left[1, e^{j \omega i}, e^{j 2 \omega i}, \ldots, e^{j(M-1) \omega i}\right]^{T} ; \quad i=1,2, \ldots, p
$$

es un conjunto de $p$ vectores linealmente independientes. Como en el caso para dos exponenciales complejos, la ecuación (3.94 se puede escribir como sigue:

$$
\mathbf{R}_{x}=\mathbf{E P} \mathbf{E}^{H}+\sigma_{w}^{2} \mathbf{I}
$$

donde $\mathbf{E}=\left[\mathbf{e}_{1}, \ldots, \mathbf{e}_{p}\right]$ es una matriz $M \times p$ que contiene $p$ vectores $\mathbf{e}_{i}$ de la señal, y $\mathbf{P}=$ $\operatorname{diag}\left\{P 1, \ldots, P_{p}\right\}$ es una matriz diagonal de las potencias de la señal. Ya que los eigenvalores de $\mathbf{R}_{x}$ son $\lambda_{i}=\lambda_{i}^{s}+\sigma_{w}^{2}$, donde $\lambda_{i}^{s}$ son los eigenvalores de $\mathbf{R}_{s}, \mathrm{y} \mathbf{R}_{s}$ es una matiz de rango $p$, entonces los primeros $p$ eigenvalores de $\mathbf{R}_{x}$ serán mayores que $\sigma_{w}^{2}$ y los últimos $M-p$ eigenvalores serán iguales a $\sigma_{w}^{2}$. Por lo tanto, los eigenvalores y eigenvectores de $\mathbf{R}_{x}$ pueden dividirse en dos grupos: los eigenvectores de la señal $\mathbf{v}_{1}, \ldots, \mathbf{v}_{p}$ y los eigenvectores del ruido $\mathbf{v}_{p+1}, \ldots, \mathbf{v}_{M}$. Asumiendo que los eigenvectores están normalizados, podemos descomponer $\mathbf{R}_{x}$ como sigue:

$$
\mathbf{R}_{x}=\sum_{i=1}^{p}\left(\lambda_{i}^{s}+\sigma_{w}^{2}\right) \mathbf{v}_{i} \mathbf{v}_{i}^{H}+\sum_{i=p+1}^{M} \sigma_{w}^{2} \mathbf{v}_{i} \mathbf{v}_{i}^{H}
$$

en notación matricial, esta descomposición puede escribirse como

$$
\mathbf{R}_{x}=\mathbf{V}_{s s} \mathbf{V}_{s}^{H}+\mathbf{V}_{n n} \mathbf{V}_{n}^{H}
$$

donde

$$
\mathbf{V}_{s}=\left[\mathbf{v}_{1}, \mathbf{v}_{2}, \ldots, \mathbf{v}_{p}\right]
$$

es la matriz $M \times p$ de los eigenvectores de la señal y

$$
\mathbf{V}_{s}=\left[\mathbf{v}_{p+1}, \mathbf{v}_{p+2}, \ldots, \mathbf{v}_{M}\right]
$$

es la matriz $M \times(M-p)$ de los eigenvectores de ruido, y donde los subíndices ${ }_{s} \mathrm{y}_{n}$ denotan las matrices diagonales que contienen los eigenvalores $\lambda_{i}=\lambda_{i}^{s}+\sigma_{w}^{2}$ y $\lambda_{i}=\sigma_{w}^{2}$, respectivamente. Como en el caso de uno y dos exponenciales complejos en ruido blanco, la ortogonalidad de los subespacios de la señal y el ruido se puede utilizar para estimar las frecuencias de los exponenciales complejos. Específicamente, debido a que cada vector de la señal $\mathbf{e}_{i}, \ldots, \mathbf{e}_{p}$ 
está en el subespacio de la señal, esta ortogonalidad implica que $\mathbf{e}_{i}$ sera ortogonal a cada eigenvector del ruido,

$$
\mathbf{e}_{i}^{H} \mathbf{v}_{k}=0 ; \quad i=1,2, \ldots, p ; \quad k=p+1, p+2, \ldots, M
$$

Por lo tanto, las frecuencias pueden ser estimadas usando una función de estimación de frecuencia como:

$$
\hat{P}\left(e^{j \omega}\right)=\frac{1}{\sum_{i=p+1}^{M} \alpha_{i}\left|\mathbf{e}^{H} \mathbf{v}_{i}\right|^{2}}
$$

\subsubsection{Multiple Signal Classification (MUSIC)}

Este método de estimación espectral fue presentado por Schmidt y es una mejora al método de descomposición armónica de Pisarenko. El algoritmo MUSIC es una técnica de estimación de frecuencia en el cual se asume que $x(n)$ es un proceso aleatorio que consiste de $p$ exponenciales complejos en ruido blanco con varianza $\sigma_{w}^{2}$. Para explicar cómo funciona este algoritmo, se asume que la matriz de autocorrelación de $x(n)$ es igual a $\mathbf{R}_{x} \mathrm{y}$ tiene una dimensión $M \times M$ con $M>(p+1)$. Si los eigenvalores de $\mathbf{R}_{x}$ se ordenan en orden decreciente $\lambda_{1} \geq \lambda_{2} \geq \cdots \geq \lambda_{M}$, y si $\mathbf{v}_{1}, \mathbf{v}_{2}, \ldots, \mathbf{v}_{M}$ son los eigenvectores correspondientes, entonces podemos dividir estos eigenvectores en dos grupos, los $p$ eigenvectores de la señal que corresponden a los $p$ mayores eigenvalores, y $M-p$ eigenvectores de ruido, que idealmente tienen los eigenvalores igual a $\sigma_{w}^{2}$. Sin embargo, con un cálculo inexacto de la secuencia de autocorrelación, los $(M-p)$ eigenvalores más pequeños serán aproximadamente iguales a $\sigma_{w}^{2}$. Sin embargo, se puede estimar la varianza del ruido blanco promediando los $(M-p)$ eigenvalores más pequeños de la siguiente forma:

$$
\hat{\sigma}_{\omega}^{2}=\frac{1}{M-p} \sum_{k=p+1}^{M} \lambda_{k}
$$

Estimar las frecuencias de los exponenciales complejos es un poco más difícil, ya que los eigenvectores de $\mathbf{R}_{x}$ son de longitud $M$, y cada eigenfiltro del subespacio de ruido

$$
V_{i}(z)=\sum_{k=0}^{M-1} v_{i}(k) z^{-k} ; \quad i=p+1, \ldots, M
$$


tendrá $(M-1)$ raíces. Idealmente, $p$ de estas raíces caerán sobre el círculo unitario a las frecuencias de los exponenciales complejos, y el eigenespectro

$$
\left|V_{i}\left(e^{j \omega}\right)\right|^{2}=\frac{1}{\left|\sum_{k=0}^{M-1} v_{i}(k) e^{-j k \omega}\right|^{2}}
$$

asociado con el eigenvector de ruido $\mathbf{v}_{i}$ exhibirá picos agudos a las frecuencias de los exponenciales complejos. Sin embargo, los $(M-p-1)$ ceros restantes pueden situarse donde sea y de hecho algunos estarán cerca del círculo unitario, provocando que los picos espurios en el eigenespectro aparezcan. Además, con autocorrelaciones inexactas, los ceros de $V_{i}(z)$ que están en el círculo unitario podrían no permanecer sobre él. Por lo tanto, cuando solo un eigenvector de ruido es usado para estimar las frecuencias de los exponenciales complejos, habrá cierta ambigüedad para distinguir los picos deseados de los espurios. Por esta razón, en el algoritmo MUSIC y para minimizar los efectos de estas frecuencias espurias que aparecen a distintas frecuencias, se hace un promedio, y se utiliza la siguiente función de estimación de frecuencia:

$$
\hat{P}_{M U}\left(e^{j \omega}\right)=\frac{1}{\sum_{i=p+1}^{M}\left|\mathbf{e}^{H} \mathbf{v}_{i}\right|^{2}}
$$

Las frecuencias de los exponenciales complejos son tomadas como la localización de los $p$

picos más grandes en $\hat{P}_{M U}\left(e^{j \omega}\right)$. Una vez que estas frecuencias son determinadas, la potencia de cada exponencial complejo se puede determinar usando:

$$
\sum_{k=1}^{p} P_{k}\left|V_{i}\left(e^{j \omega k}\right)\right|^{2}=\lambda_{i}-\sigma_{\omega}^{2} ; \quad i=1,2, \ldots, p
$$

\subsection{Métodos de descomposición empírica}

El método de descomposición de modos empírico (EMD, acrónimo de Empirical Mode Decomposition) fue propuesto por Huang et al. 101 para lidiar con datos no estacionarios y no lineales. Según sus creadores, este método es intuitivo, directo, a posteriori y adaptativo con el fundamento de que la descomposición está basada y derivada de los datos. La EMD se basa en tres hipótesis: 1) la señal tiene al menos dos extremos, es decir un máximo y un mínimo; 2) la escala de tiempo característica está definida por el lapso de tiempo entre los 
extremos y 3) si los datos carecen de extremos, pero contienen puntos de inflexión, entonces pueden diferenciarse una o más veces para revelar los extremos. Los resultados finales pueden obtenerse mediante la integración o integraciones de los componentes. La esencia de este método es la de identificar empíricamente en los datos los modos intrínsecos de oscilación por sus escalas de tiempo características y, a partir de ellas, descomponer la señal. Los autores definen la escala de tiempo como el lapso de tiempo entre dos extremos sucesivos. El método sistemático para extraer los modos de oscilación se explica a continuación. Primero, el método de descomposición usa las envolventes separadamente definidas por los máximos y los mínimos locales. Para hacer esto, todos los máximos locales se unen por una interpolación cúbica, generando la envolvente superior. Lo mismo se hace con los mínimos locales para generar la envolvente inferior. Estas dos envolventes deben cubrir todos los datos entre ellas. La media entre las dos envolventes se designa como $m_{1}$, y la diferencia entre los datos y $m_{1}$ es el primer componente $h_{1}$.

$$
h_{1}=x(t)-m_{1}
$$

En el segundo paso, el proceso de suavizado se repite más veces. En el segundo proceso, $h_{1}$ es ahora tratado como si fuese los datos en el primer punto, entonces

$$
h_{11}=h_{1}-m_{11}
$$

y este proceso se repetirá $k$ veces,

$$
h_{1 k}=h_{1(k-1)}-m_{1 k}
$$

hasta que en $h_{1 k}$ la suma de máximos y mínimos, y el número de cruces por cero sean iguales o distintos en un valor. Además de que en cualquier punto de $h_{1 k}$, el valor medio de la envolvente definida por el máximo local y la envolvente definida por el mínimo local, sea cero. Entonces $h_{1 k}$ se puede considerar como una función de modo intrínseco (IMF, acrónimo de Intrinsic Mode Function), es decir:

$$
c_{1}=h_{1 k}
$$

donde $c_{1}$ será la primera IMF de los datos. 
Para asegurar que la IMF contenga suficiente sentido físico en amplitud y modulación, es necesario determinar un criterio para detener el proceso de cribado. Esto se puede realizar limitando el tamaño de la desviación estándar, SD, calculada a partir de dos procesos consecutivos, es decir

$$
S D=\sum_{t=0}^{T}\left[\frac{\left|\left(h_{1(k-1)}(t)-h_{1 k}(t)\right)\right|^{2}}{h_{1(k-1)}^{2}(t)}\right]
$$

Un valor típico de la SD para detener el proceso de cribado estará entre 0.2 y 0.3. Seguido de la obtención de la primera IMF, donde $c_{1}$ contiene el componente de la señal de mas alta frecuencia, se debe separar $c_{1}$ del resto de los datos,

$$
r_{1}=x(t)-c_{1}
$$

Debido a que el residuo $r_{1}$, contiene información de componentes de menor frecuencia que el componente $c_{1}, r_{1}$ será ahora tratado como los nuevos datos y se iniciará el proceso nuevamente hasta obtener el componente $c_{2}$. Este procedimiento se repetirá con todos los residuos subsecuentes,

$$
r_{n}=r_{n-1}-c_{n}, \ldots, r_{2}=r_{1}-c_{2}
$$

Este proceso de iteración se deberá detener por alguno de los siguientes criterios: cuando el componente $c_{n}$ o el residuo $r_{n}$ sean tan o más pequeños que un valor predeterminado o cuando el residuo $r_{n}$ sea una función monótona de la cual no se puedan extraer más IMFs. A partir de las IMFs obtenidas y el residuo final se puede reconstruir la señal original,

$$
x(t)=\sum_{i=1}^{n} c_{i}+r_{n}
$$

donde $c_{i}$ son los componentes de la señal y $r_{n}$ es el residuo, que puede ser la tendencia media o una constante. Sin embargo esta reconstrucción no es exacta debido a los efectos del ruido y además se presenta mezclado de modos.

La EMD es un método de análisis que ha probado ser muy versátil en una amplia gama de aplicaciones para extraer señales de datos obtenidos de procesos no lineales y no estacionarios con ruido. Sin embargo, el método tiene algunos inconvenientes. El mayor de estos 
inconvenientes es la frecuente aparición de mezclado de modos [102], la cual se manifiesta en una sola IMF, en la cual confluyen señales con escalas u oscilaciones ampliamente dispares, o también se manifiesta como la presencia de una misma señal en distintos componentes IMF. El mezclado de modos es a menudo una consecuencia de la intermitencia de la señal. Para resolver el problema de la separación de escalas, se propuso el EEMD (acrónimo de Ensemble Empirical Mode Decomposition), el cual define los componentes IMF verdaderos como el promedio de un conjunto de ensayos, donde cada ensayo consiste de la señal más ruido blanco de amplitud finita. De manera general, todos los datos que se registran de un fenómeno físico son una amalgama de la señal deseada y ruido, es decir,

$$
x(n)=s(n)+n(n)
$$

donde $x(n)$ son los datos capturados, $s(n)$ es la señal deseada y $n(n)$ es el ruido. Para mejorar la precisión de las medidas registradas, el promedio de conjuntos es una poderosa forma de hacerlo. Para lograr esto, los datos son capturados en observaciones separadas, cada una de las cuales tendrá distinto contenido de ruido. Para implementar esta idea, en el método EEMD se introduce ruido en los datos de la señal, $x(n)$, como si se tratara de distintas observaciones. Este proceso será análogo a realizar un gran número de ensayos experimentales. El ruido blanco que se adiciona a la señal analizada debe tener características de aleatoriedad similares a las que contiene el proceso que se esté analizando. Bajo estas condiciones, la iésima observación artificial será:

$$
x_{i}(n)=x(n)+w_{i}(n)
$$

En el caso de una sola observación, se agregarán distintas realizaciones de ruido las cuales serán no arbitrarias. A pesar de que agregar ruido disminuye la relación señal/ruido, el ruido blanco agregado proporciona una relativamente uniforme distribución de la referencia de escala que facilita la EMD. Por lo tanto, la baja relación señal-a-ruido no afecta el método de descomposición, y de hecho lo mejora al evitar el mezclado de modos. En base a esto el método EEMD se implementa como sigue [103].

- Se agrega ruido a los datos que se pretenden analizar. 
- Se hace la descomposición de los datos en IMFs como se haría con el método EMD.

- Se repiten los pasos 1 y 2 un gran número de veces, pero con diferentes realizaciones de ruido blanco cada vez.

- Por último, se obtiene el promedio de las correspondientes IMFs de cada descomposición como resultado final.

Los efectos de la descomposición usando la EEMD son que el ruido blanco añadido se cancela en el promedio de las correspondientes IMFs. Para asegurar que la descomposición sea correcta y que el ruido blanco agregado sea eliminado durante el promediado y que solo permanecerá la señal original se deben realizar un número suficiente de realizaciones. A pesar de que este algoritmo es una mejora sustancial a la EMD, se crean nuevos problemas como el hecho de que la reconstrucción de la señal de las IMFs obtenidas contendrá ruido residual, además de que distintas realizaciones de la descomposición generaran diferentes números de modos o IMFs.

\subsubsection{CEEMD}

Para resolver los problemas que se presentan en la EMD y en la EEMD, Torres et al. 104 propusieron la CEEMD (acrónimo de Complete Ensemble Empirical Mode Decomposition). En este nuevo algoritmo, el ruido es agregado en cada paso de descomposición, y la descomposición final está basada en un solo residuo para obtener cada modo. Las mejoras que se dan en este algoritmo en comparación con la EMD y la EEMD, son que la reconstrucción de la señal es completa, la descomposición de la señal produce un número menor de modos que en la EEMD, y el número de iteraciones se reduce a la mitad comparada con la EEMD. La CEEMD es un método asistido por ruido que tiene el siguiente procedimiento:

Primero, se agrega ruido blanco gaussiano a los datos que se analizaran con el fin de obtener la primera descomposición de los datos con ruido. La descomposición se repite I veces usando diferentes realizaciones de ruido. Entonces, se calcula el promedio del conjunto, el cual definirá la primera IMF de los datos analizados. Entonces, 


$$
I M F_{1}=\frac{1}{I} \sum_{i=1}^{I} E_{1}\left[x(t)+\varepsilon w_{i}\right]
$$

donde, $I M F_{1}$ es el primer componente de los datos $x(n), w_{i}$ es el ruido blanco gaussiano de media cero con varianza unitaria, $\varepsilon$ es un coeficiente de ajuste, $E_{1}$ produce el i-ésimo componente IMF e I es el número de realizaciones. Entonces el primer residuo $r_{1}$ de la señal se calcula como sigue,

$$
r_{1}=x(t)-I M F_{1}
$$

después, se llevan a cabo las realizaciones de descomposición $r_{1}+\varepsilon E_{1}\left[w_{i}\right], i=1,2, \ldots, I$ hasta que alcancen las condiciones de la primera IMF y se define el promedio de los conjuntos como la segunda función de modo intrínseco, $I M F_{2}$.

$$
I M F_{2}=\frac{1}{I} \sum_{i=1}^{I} E_{2}\left[r_{1}+\varepsilon E_{1}\left[w_{i}\right]\right]
$$

para $k=2,3, . ., K$, el k-ésimo residuo se calcula como: $r_{k}=r_{k-1}-I M F_{k}$, entonces el primer componente $I M F$ de $r_{k}+\varepsilon E_{k}\left[w_{i}\right], I=1,2, \ldots, I$ es extraído y el promedio es calculado una vez más para obtener $I M F_{(k+1)}$ de la señal:

$$
I M F_{k+1}=\frac{1}{I} \sum_{i=1}^{I} E_{k+1}\left[r_{k}+\varepsilon E_{k}\left[w_{i}\right]\right]
$$

El proceso de suavizado continúa hasta que el último residuo no tenga más de dos extremos, produciendo

$$
R=x(t)-\sum_{k=1}^{K} I M F_{K}
$$

donde $R$ es el residuo final y $\mathrm{K}$ es el número total de IMFs. Por lo tanto, la señal original puede expresarse como

$$
x(t)=\sum_{k=1}^{K} I M F_{K}+R
$$

La ecuación anterior define a la CEEMD como un método de descomposición completa, y comparada con la EMD y la EEMD, la CEEMD no solo resuelve el problema del mezclado de modos, sino que además proporciona una reconstrucción exacta de la señal original [105]. 


\section{Capítulo 4}

\section{Experimentación}

\subsection{Análisis en estado estable}

En esta sección se presenta la metodología propuesta para el análisis de las señales provenientes de un motor durante el funcionamiento en estado estable, la validación de la metodología propuesta y la experimentación para la captura de las señales de sonido y vibraciones.

\subsubsection{Metodología}

La Figura 4.1 muestra la metodología seguida para determinar la condición del motor y para evaluar la factibilidad del método propuesto. En primer lugar, en el diagrama de bloques, se presenta la implementación de un banco de pruebas con varios motores de inducción con diferentes casos de estudio: motor sano, motor con dos barras del rotor rotas, defecto en la pista externa del rodamiento y desbalance en la polea. El siguiente paso es la adquisición de señales de vibración y sonido. Las señales de vibración son adquiridas con un acelerómetro triaxial y el sonido con un micrófono. Las señales son capturadas durante la operación del motor en estado estacionario. Las señales de vibración se capturan a una frecuencia de muestreo de $1.5 \mathrm{kHz}$ para un tiempo de adquisición de 2.73 s para obtener 4,096 muestras y la señal de sonido a una frecuencia de muestreo de $2 \mathrm{kHz}$ durante 8.192 segundos para obtener 16,384 muestras. Una vez completado el proceso de adquisición y almacenamiento de señales, se procesan y analizan las señales adquiridas. En esta etapa, se realiza una descomposición 
en tiempo a través de la CEEMD y posteriormente los IMFs obtenidos se transforman al dominio de la frecuencia. Esta transformación al dominio de la frecuencia se realiza con el cálculo del marginal en frecuencia de la representación tiempo-frecuencia de Gabor (TFDG). Finalmente, una vez obtenidos los espectros de los IMFs, se evalúa la condición del motor analizando las frecuencias relacionadas con las fallas y se hace una comparación con trabajos previos para validar la efectividad de la metodología propuesta.

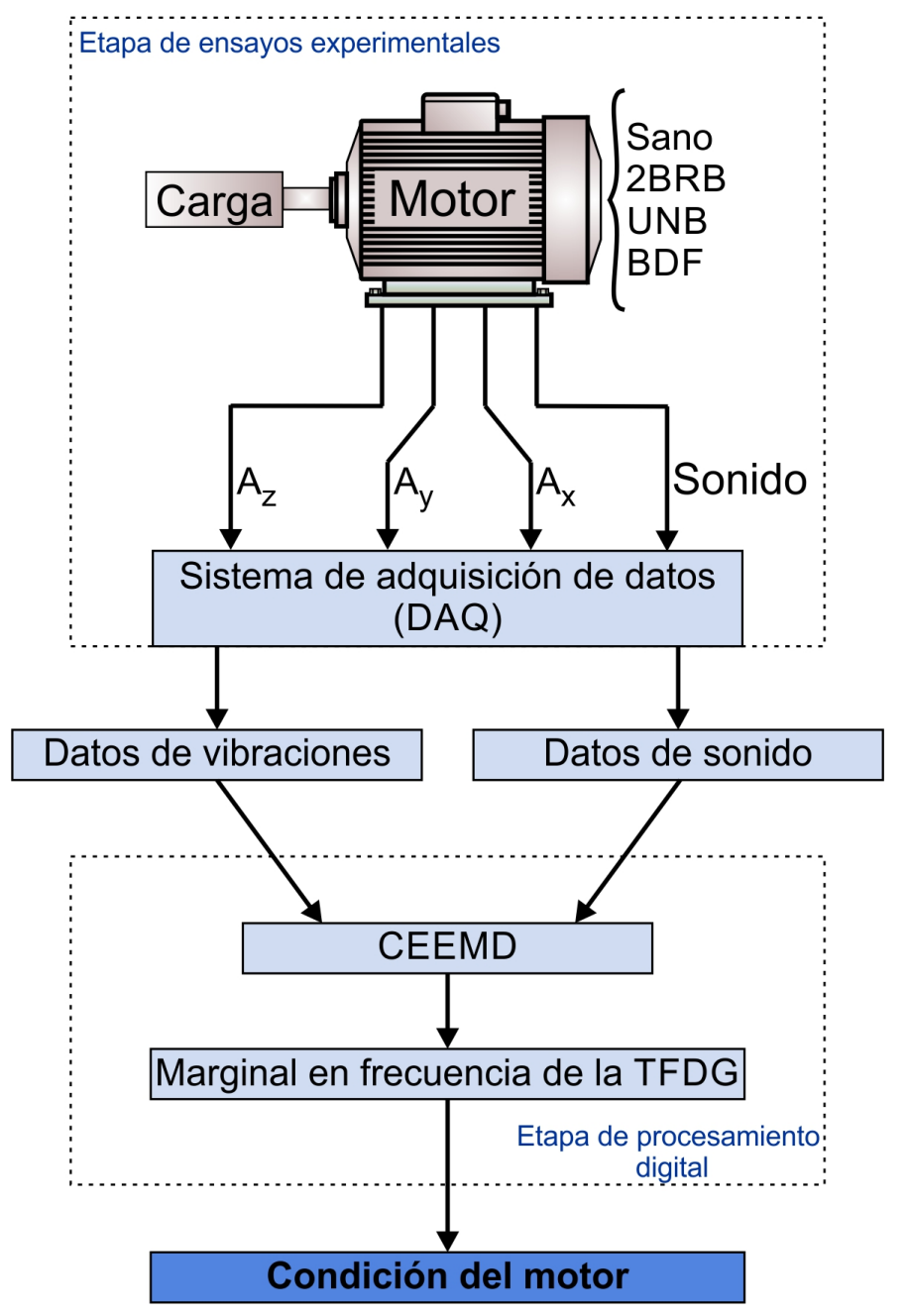

Figura 4.1: Metodología propuesta para el estado estable. 


\subsubsection{Validación de la metodología propuesta}

Esta sección presenta una comparación de efectividad entre la metodología propuesta en este trabajo y la transformada de Fourier, que es la técnica clásica para el análisis espectral de señales estacionarias. Se analiza una señal sintética para validar la metodología propuesta. Esta señal tiene características similares a las señales obtenidas de un motor de inducción durante la operación en estado estacionario, y está compuesta de un conjunto de sinusoides puros de la misma amplitud y con frecuencias constantes iguales a 60, 200, 780 y $800 \mathrm{~Hz}$, respectivamente. Se genera con una frecuencia de muestreo de $f_{s}=2000 \mathrm{~Hz}$ y una duración de 4 segundos. Finalmente, se añade ruido blanco gaussiano $\left(n_{G}\right)$ a la señal para emular el contenido de ruido de las señales capturadas de un motor real. Esto ayudará a estudiar el comportamiento de la metodología con señales con una baja relación señal/ruido. La relación señal/ruido de la señal sintética utilizada corresponde a $S N R=-15 d B$. La ecuación (4.1) define la señal sintética generada:

$$
x(k)=\sin \left(2 \pi f_{1} k\right)+\sin \left(2 \pi f_{2} k\right)+\sin \left(2 \pi f_{3} k\right)+\sin \left(2 \pi f_{4} k\right)+n_{G}
$$

donde $f_{1}=60 \mathrm{~Hz}, f_{2}=200 \mathrm{~Hz}, f_{3}=780 \mathrm{~Hz}, f_{4}=800 \mathrm{~Hz}$ y $k=n / f_{s}$ para $n=0,1, \ldots 8000$ es el tiempo discreto. En la Figura 4.2 (a), se muestra la señal sintética generada. En la figura 4.2 (b), se muestra el espectro de señal ideal. La Figura 4.2 (c) muestra el espectro de la señal obtenida con la transformada rápida de Fourier. En la Figura 4.2 (d), se muestra el espectro obtenido tras el cálculo de la marginal en frecuencia de la distribución de Gabor a partir de los IMFs seleccionados de acuerdo a las frecuencias de interés. En este caso, el IMF 1 se selecciona para identificar funciones sinusoidales con frecuencias de 780 y $800 \mathrm{~Hz}$, el IMF 3 para el componente de $200 \mathrm{~Hz}$ y el IMF 5 para el componente de $60 \mathrm{~Hz}$. Como se muestra en la Figura 1 (d), los componentes espectrales relacionados con las cuatro funciones seno pueden identificarse claramente utilizando la metodología propuesta en este trabajo, a diferencia del espectro obtenido con la FFT en el que no es posible discernir qué componentes espectrales pertenecen al ruido contenido en la señal de los procedentes de los componentes de la función sinusoidal. 


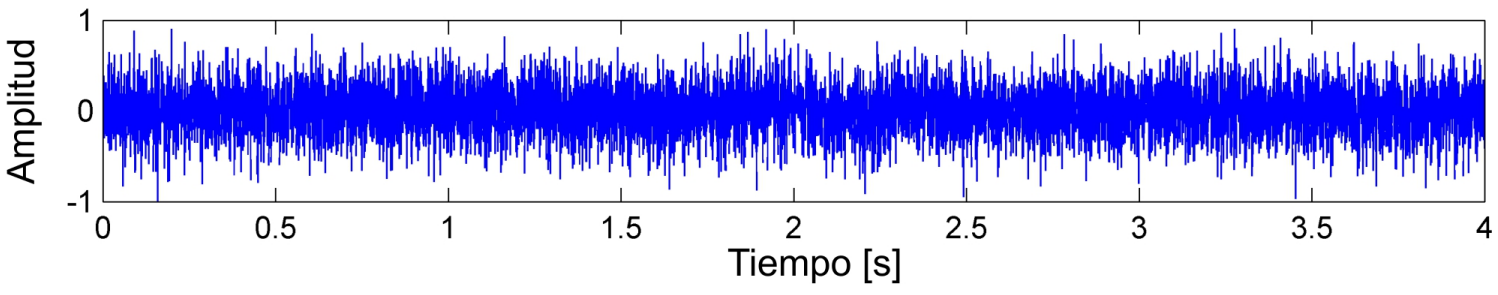

(a)
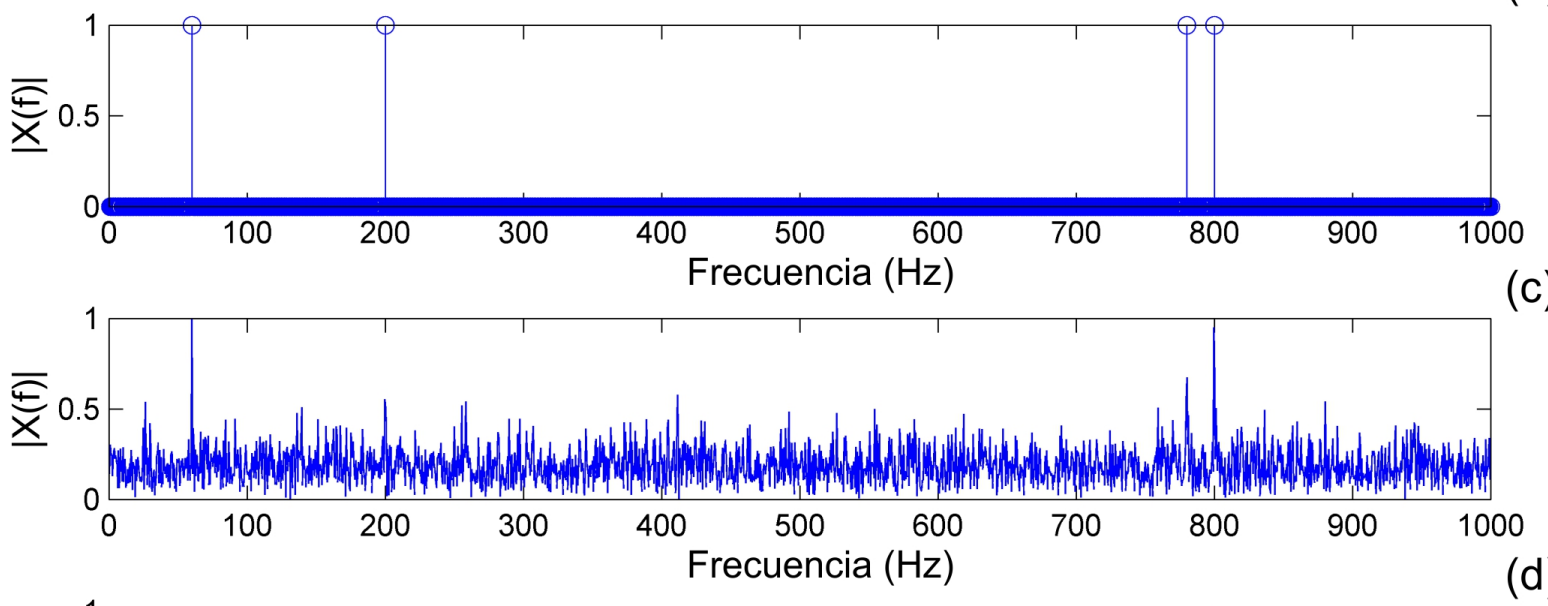

(d)

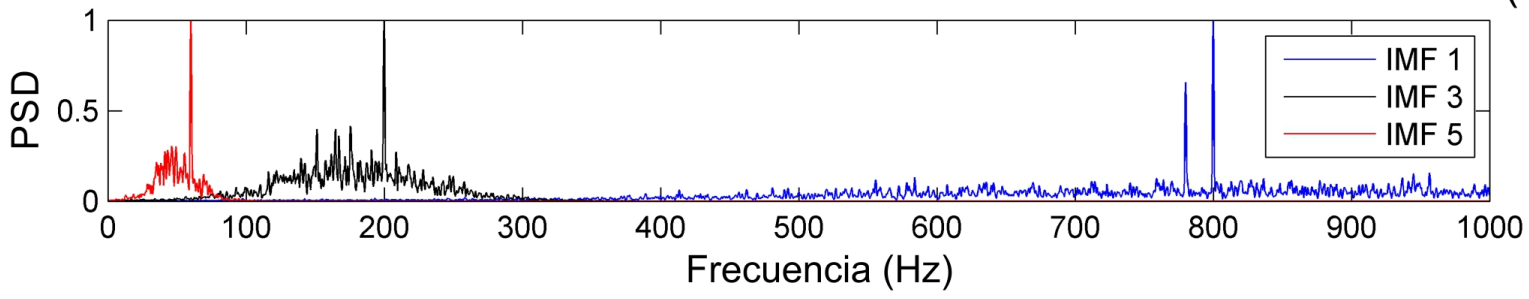

Figura 4.2: Comparación entre la metodología propuesta y la FFT. a) Señal sintética. b) Espectro ideal. c) FFT de la señal. d) Marginal en frecuencia de la TFDG de las IMFs seleccionadas

La elección de los IMFs no suele hacerse a priori debido a las características de la CEEMD, en la que las bandas o modos de descomposición no coinciden con bandas predeterminadas, como en el caso de las Wavelets. Los diferentes modos en que se descompone la señal son variables, adaptativos y totalmente dependientes de la señal [101] Así, basándose en este hecho, la mejor manera de seleccionar los modos de interés es elegirlos a posteriori, haciendo 
un análisis de todos los IMFs resultantes de la descomposición de la señal. Afortunadamente, hay alguna guía para encontrar la frecuencia de interés y para seleccionar un modo particular. El primer IMF contiene las oscilaciones más rápidas (las componentes de la señal de las frecuencias más altas) y el último IMF las oscilaciones más lentas (las componentes de la señal de las frecuencias más bajas).

\subsubsection{Experimentación}

Durante la experimentación en estado estable se captura una señal de sonido para identificar las características dinámicas del motor de inducción. También se obtienen señales de vibración para verificar los resultados obtenidos. Se hacen cuarenta pruebas para el sonido y las vibraciones en cada condición con el fin de validar la metodología propuesta. La Figura 4.3 muestra la configuración del experimento donde se usan diferentes motores de inducción trifásicos (modelo WEG 00136APE48T, 745.7 Watts) para probar el desempeño de la metodología propuesta que identifica las condiciones de falla tratadas en este trabajo. Los motores probados tienen dos polos, 28 barras y se alimentan con una fuente de alimentación de 220 VAC a $60 \mathrm{~Hz}$. La carga mecánica aplicada es la de un alternador ordinario. La señal de sonido se adquiere utilizando un micrófono de condensador modelo JST CX-509, que tiene un patrón de polarización de cardioide. El micrófono se coloca en una posición conveniente donde las vibraciones del motor no tienen ninguna influencia en el micrófono. Por otra parte, la señal de vibración se adquiere usando un acelerómetro triaxial basado en MEMS (modelo LIS3L02AS4) de STMicroelectronics colocado como se muestra en la Figura 4.3 (a). A partir de los tres ejes de aceleración $(x, y$ y $z$ ) de la señal de vibración, se obtienen los mejores resultados con la señal correspondiente al eje $z(\mathrm{Az})$. La señal de audio se amplifica utilizando un amplificador de potencia modelo Marshall MG15CDR. Un convertidor analógico-digital (ADC) de 12 bits de 4 canales en serie de salida (ADS7841) se utiliza para la adquisición de datos de sonido y vibraciones. El sistema de instrumentación utiliza una frecuencia de muestreo de $2 \mathrm{kHz}$ para obtener 16,384 muestras de sonido mientras que se obtienen 4,096 muestras a una frecuencia de muestreo de 1,500 Hz para las señales de vibración durante el estado estacionario del motor de inducción. Las figuras 4.3 (b) y 4.3 (c) muestran la orien- 
tación de los ejes de vibración en el acelerómetro. En este trabajo se tratan tres condiciones de falla diferentes que se explican a continuación.

\section{Barras rotas en el rotor de jaula de ardilla}

La condición de dos barras rotas se produce artificialmente perforando dos agujeros de $7.938 \mathrm{~mm}$ de diámetro sin dañar el eje del rotor. La Figura 4.4 muestra el rotor utilizado con dos barras de rotor rotas. El motor funciona a 3,465 RPM en un sistema de $60 \mathrm{~Hz}$; el motor de inducción ensayado tiene una frecuencia del rotor $f r=57.75 \mathrm{~Hz}$. Por lo tanto, el deslizamiento $s$ es 0.0375 y las frecuencias de banda lateral asociadas con la barra rota del rotor se calculan utilizando la ecuación (2.7).

\section{Desbalance}

La condición de desbalance está presente cuando la carga mecánica del motor de inducción no está distribuida uniformemente, desplazando el centro de masa del eje del motor. La Figura 4.5 muestra una polea con una masa añadida usada para generar desbalance en el motor de inducción. La firma del desequilibrio en una señal de vibración normalmente tiene la forma de amplitud incrementada a lo largo de la frecuencia del rotor, estando situada en este caso a $57.75 \mathrm{~Hz}$.

\section{Defectos en el rodamiento}

Para realizar la prueba del rodamiento con defectos, el rodamiento se daña artificialmente perforando dos agujeros con $1.191 \mathrm{~mm}$ de diámetro en su pista exterior usando una broca de tungsteno. La Figura 4.6 muestra el rodamiento 6203-2ZNR utilizado en este trabajo. La frecuencia característica del defecto en el espectro de vibraciones de la pista exterior que soporta el elemento rodante se calcula utilizando la ecuación (2.8). El motor de inducción ensayado tiene una frecuencia de rotación del rotor $f r=57.75 \mathrm{~Hz}$ y el rodamiento de prueba tiene ocho bolas con un diámetro de $6.5 \mathrm{~mm}$ y el diámetro de la jaula del rodamiento es de 28 $\mathrm{mm}$ con un ángulo de contacto $\theta=0$, por lo tanto, la frecuencia de fallo de la pista externa del rodamiento $\left(f_{B P O F}\right)$ se localiza a $177.7 \mathrm{~Hz}$.

UG - UVa

Paulo Antonio Delgado Arredondo 


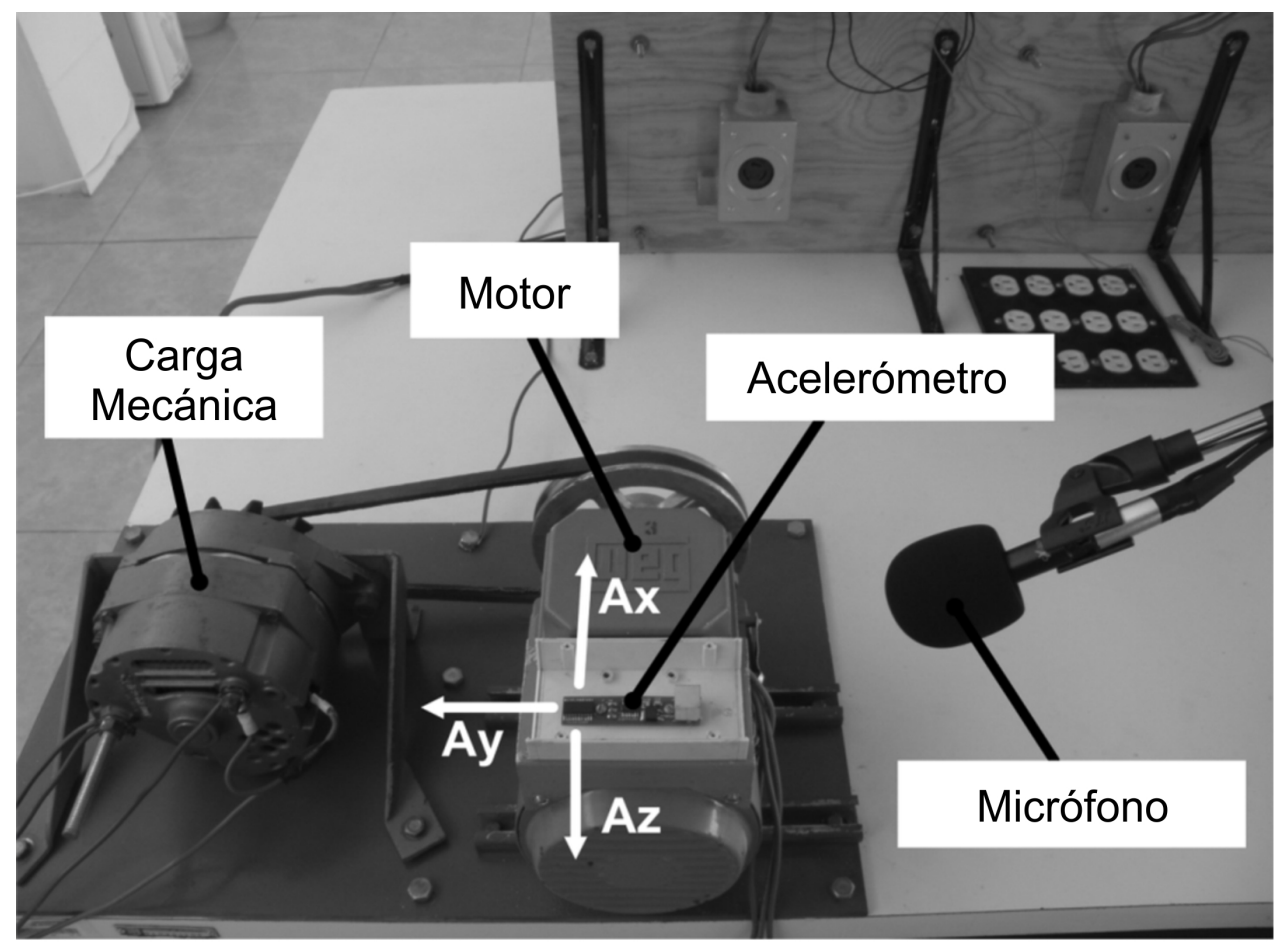

a)

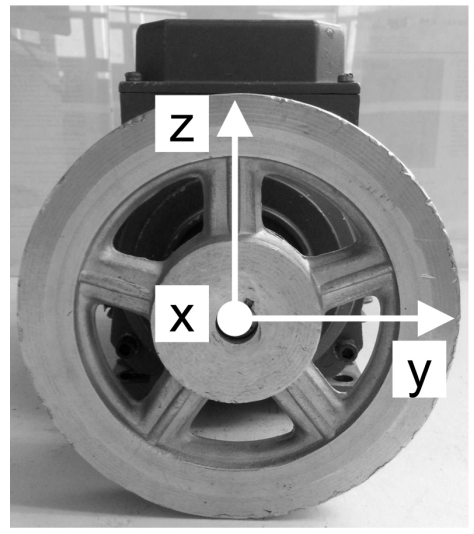

b)

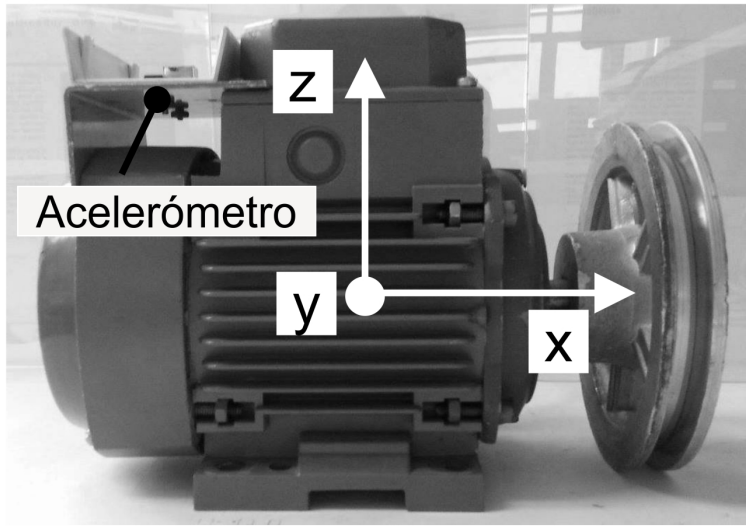

c)

Figura 4.3: Banco de pruebas usado para los ensayos en estado estable. a) Vista general. b) Vista frontal del motor donde se muestra la orientación de los ejes del acelerómetro. c) Vista lateral del motor donde se muestra la orientación de los ejes del acelerómetro. 


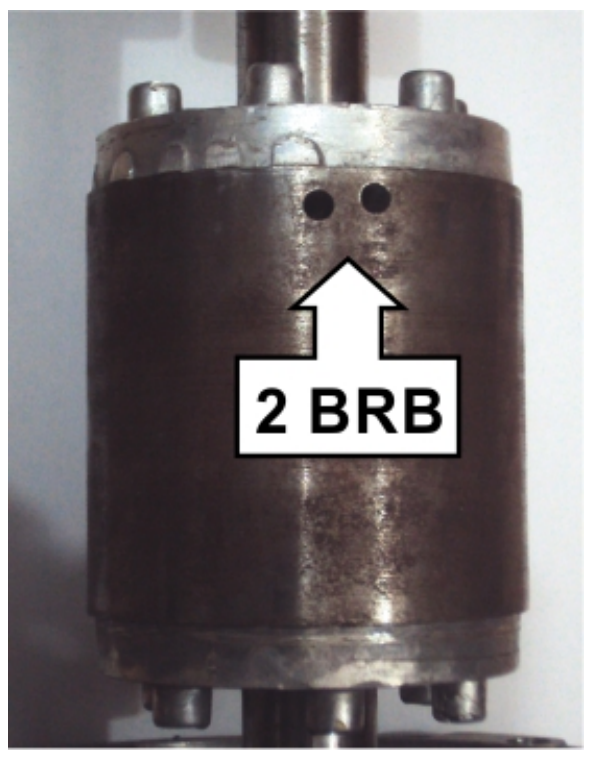

Figura 4.4: Rotor con 2 barras rotas.

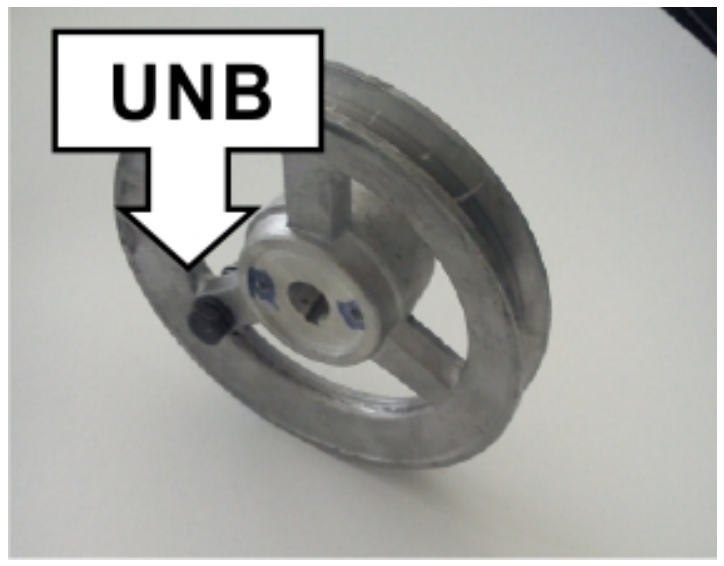

Figura 4.5: Polea con desbalance. 


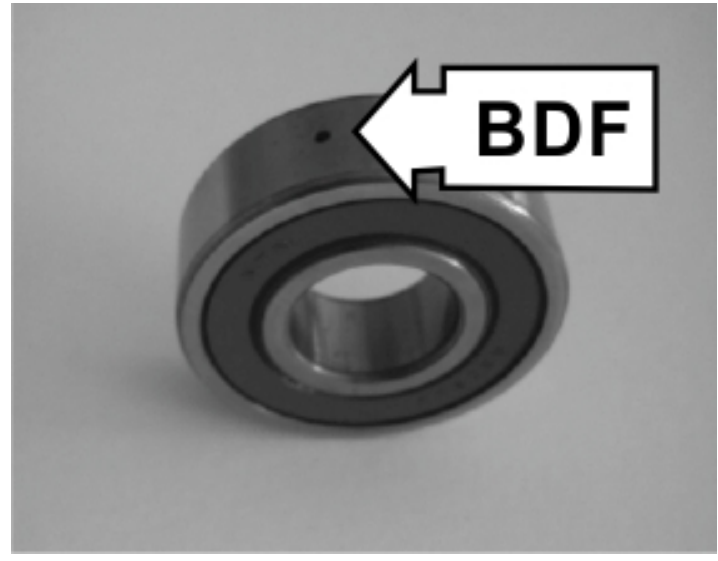

Figura 4.6: Rodamiento dañado. 


\subsection{Análisis en el transitorio de arranque}

En esta sección se presenta la metodología propuesta para el análisis de las señales provenientes de un motor durante el transitorio de arranque, la validación de la metodología propuesta y la experimentación para la captura de las señales de vibraciones.

\subsubsection{Metodología}

La Figura 4.7 representa un diagrama de bloques de la metodología propuesta para la captura y análisis de las señales de vibración de un motor de inducción durante el transitorio de arranque. En primer lugar, el banco de pruebas se ajusta con el motor de inducción en diferentes condiciones: sano, una y dos barras del rotor rotas, desbalance y defecto en la pista exterior del rodamiento. A continuación, se inicia el proceso de adquisición de datos de las vibraciones a través del acelerómetro triaxial, esto durante el transitorio de arranque. Durante el transitorio de arranque, se obtienen 4,096 muestras a $1.5 \mathrm{kHz}$ durante un tiempo de adquisición de 2.73 s. Una vez completado el proceso de adquisición del transitorio de arranque, se

procesan y analizan las señales de vibración adquiridas. En esta etapa se aplican las metodologías de descomposición tiempo-frecuencia TFDG, TFMS y MUSIC, y se comparan con la STFT. Finalmente, a partir de los resultados de las descomposiciones tiempo-frecuencia se determina la condición del motor. Asimismo, se realiza una evaluación cualitativa y cuantitativa de las distribuciones utilizadas para evaluar su desempeño, en comparación con la STFT.

\subsubsection{Validación de la metodología propuesta}

Para validar la metodología propuesta se genera una señal sintética como se indica en la ecuación (4.2) con el objetivo de emular componentes espectrales presentes en las señales de vibración reales del transitorio de arranque de un motor eléctrico. La señal sintética tiene tres señales sinusoidales puras con frecuencia constante a 80, 670 y $700 \mathrm{~Hz}$, más una señal sinusoidal con una frecuencia variable que oscila entre $0 \mathrm{~Hz}$ y $56.7 \mathrm{~Hz}$ de 0 a 2 s y luego permanece con una frecuencia constante. También se añade ruido blanco gaussiano a la señal. 


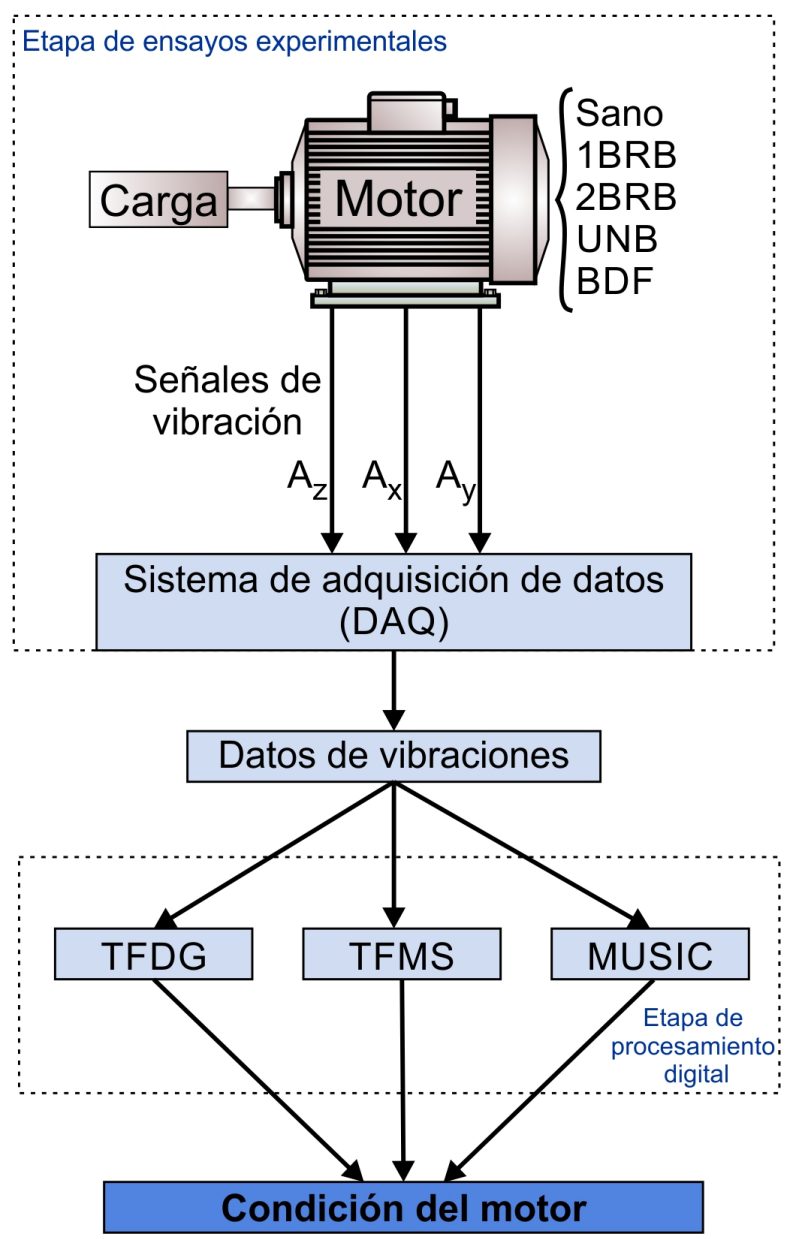

Figura 4.7: Metodología propuesta para el transitorio de arranque.

Los componentes de frecuencia constante situados en 670 y $700 \mathrm{~Hz}$ se utilizan para evaluar el rendimiento del método para discriminar componentes cercanos. La sinusoide con frecuencia variable emula un transitorio de arranque que alcanza el estado estacionario a $56.7 \mathrm{~Hz}$, que se encuentra cerca de una componente de frecuencia constante a $80 \mathrm{~Hz}$. Finalmente, se añade ruido gaussiano $\left(n_{G}\right)$ para evaluar el comportamiento del método propuesto en señales con una relación señal/ruido (SNR) baja. La señal sintética se cuantifica a una frecuencia de muestreo de $1.5 \mathrm{kHz}$, que comprende 4,096 muestras para un tiempo de funcionamiento total 
de 2.73 segundos. También la señal sintética tiene una $S N R=-3.6 d B$.

$$
\begin{aligned}
& x(k)=\sin \left(\omega_{1} k\right)+\sin \left(\omega_{2} k\right)+\sin \left(\omega_{3} k\right)+\sin \left(\omega_{4} k\right)+n_{G}, \\
& \omega_{h}=2 \pi f_{h}, \quad \text { para } h=1,2,3,4
\end{aligned}
$$

donde $x(k)$ es la señal sintética, $f_{1}=80, f_{2}=670, f_{3}=700$ y

$$
f_{4}=\left\{\begin{array}{cc}
\frac{56.7}{2} k & 0 \leq k \leq 2 \\
56.7 & 2<k
\end{array}\right.
$$

La Figura 4.8 muestra los resultados del proceso de validación para la señal sintética en el dominio del tiempo y la descomposición tiempo-frecuencia obtenida con los métodos tratados. En la Figura 4.8 a) se representa la señal sintética en el dominio del tiempo. La descomposición tiempo-frecuencia teórica se muestra en la Figura 4.8 b). Las descomposiciones tiempo-frecuencia de los métodos tratados se representan en la Figura 4.8 c) para la STFT, Figura 4.8d) para la TFDG, Figura 4.8 e) para la TFMS, y Figura 4.8f) para MUSIC.

Como se puede ver en la Figura 4.8 c), el comportamiento de la STFT con una ventana de 128 datos, muestra que no es un buen método para la descomposición tiempo-frecuencia cuando la relación señal-a-ruido es baja. También es muy difícil distinguir las dos frecuencias cercanas en 670 y $700 \mathrm{~Hz}$, mientras que la sinusoide de frecuencia variable es apenas visible y en el estado estacionario es indistinguible del componente espectral de $80 \mathrm{~Hz}$. Por otra parte, los otros métodos propuestos son capaces de distinguir claramente los componentes espectrales del ruido de fondo.

\subsubsection{Configuración experimental}

En la Figura 4.9 se muestra el banco de pruebas utilizado para realizar pruebas a los motores con diferentes tipos de fallas y el sistema de adquisición de datos utilizado para capturar las señales. Se utilizan varios motores de inducción de jaula de ardilla, modelo WEG 00136APE48T, de 1hp (745.7 W) para ensayar las condiciones sanas y defectuosas tratadas en el motor. Los motores tienen dos polos, 28 barras, se alimentan con una tensión de 220 VAC a $60 \mathrm{~Hz}$, y la carga aplicada es un alternador convencional. Las señales de vibración 
son adquiridas utilizando un acelerómetro triaxial basado en sistemas microelectromecánicos (modelo LIS3L02AS4) de STMicroelectronics. Para la adquisición de datos (DAS) se utiliza un convertidor analógico-digital de 12 bits de cuatro canales (ADS7841). Este sistema de adquisición de datos utiliza una frecuencia de muestreo $f_{s}$ de $1.5 \mathrm{kHz}$ y se obtienen 4,096 muestras en 2.73 segundos durante el transitorio de arranque.

\section{Barras rotas en el rotor de jaula de ardilla}

Como ya se ha mencionado anteriormente, las frecuencias especificas a las barras del rotor rotas dependen de la oscilación de velocidad con frecuencia $2 s f_{s}$. La condición de una y dos barras rotas se produce de igual forma a lo descrito anteriormente, haciendo una perforación en ellas. La Figura 4.10 muestra el rotor utilizado con una barra del rotor rota y en la Figura 4.4 se muestra el rotor con dos barras rotas. El motor funciona a 3,402 rpm en un sistema de

$60 \mathrm{~Hz}$. Los armónicos, específicos del fallo de barras rotas, se obtienen incorporando primero los valores reales de velocidad del motor en (2.4) para encontrar el valor de deslizamiento $(\mathrm{s}=$ 0.055) que se puede utilizar en (2.7) para obtener las frecuencias y sus armónicos relacionados con esta falla.

\section{Desbalance}

Al igual como sucede con el caso de desbalance mostrado en la sección de análisis en estado estable, en el transitorio de arranque, el desbalance se manifestará a una frecuencia igual a la frecuencia de rotación del rotor, en este caso la frecuencia de rotación del rotor alcanza un valor igual a $56.7 \mathrm{~Hz}$ hacia el final de los $2.73 \mathrm{~s}$ de duración de la captura de las señales. El desbalance generado en la polea se muestra en la Figura 4.5.

\section{Defectos en el rodamiento}

Para llevar a cabo esta falla y generar un defecto en la pista externa del rodamiento se procede igual que en el caso del análisis en estado estable. La Figura 4.6 muestra el rodamiento utilizado en los ensayos con esta falla. En este caso la velocidad de rotación del rotor es igual $56.7 \mathrm{~Hz}$, sustituyendo este valor y los valores de la geometría del rodamiento en la ecuación 
(2.8) se obtiene que la frecuencia asociada al defecto en la pista externa del rodamiento se manifestará a $174.1 \mathrm{~Hz}$ o sus armónicos. 


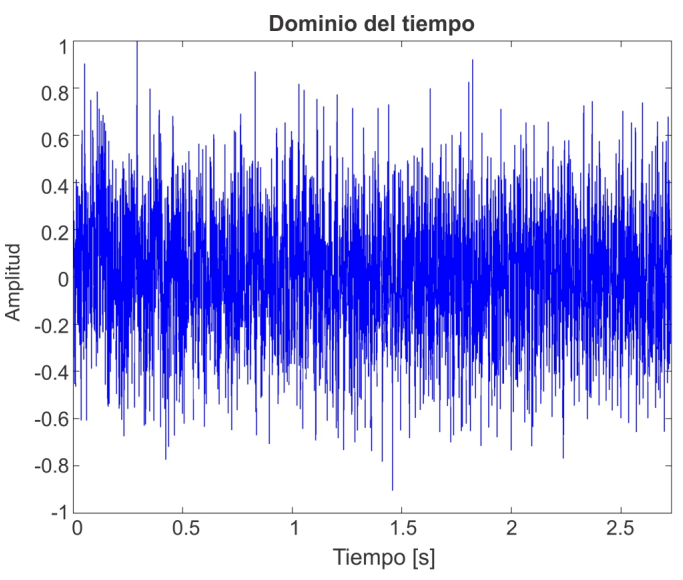

a)

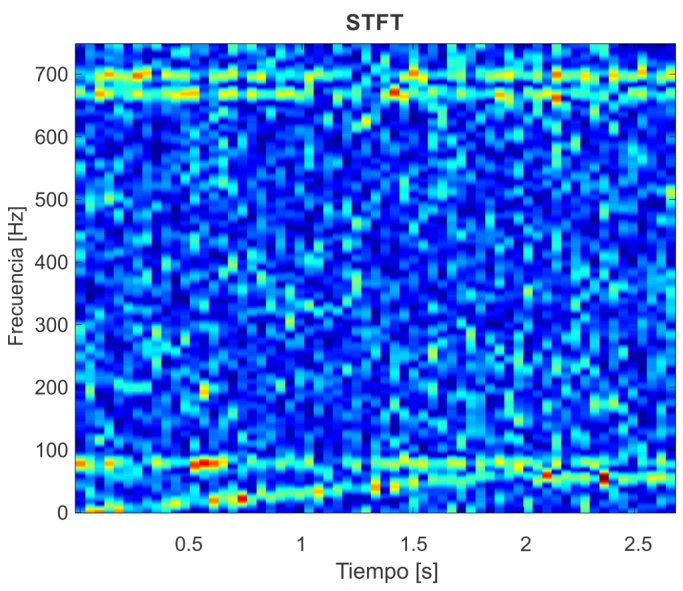

c)

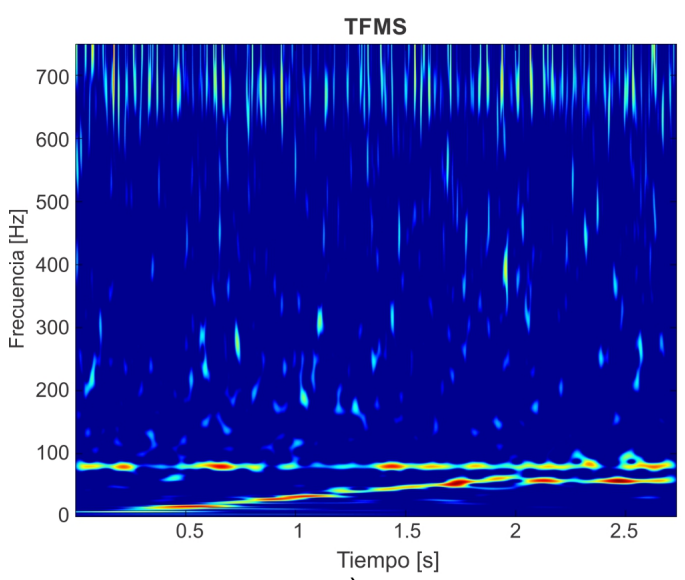

e)

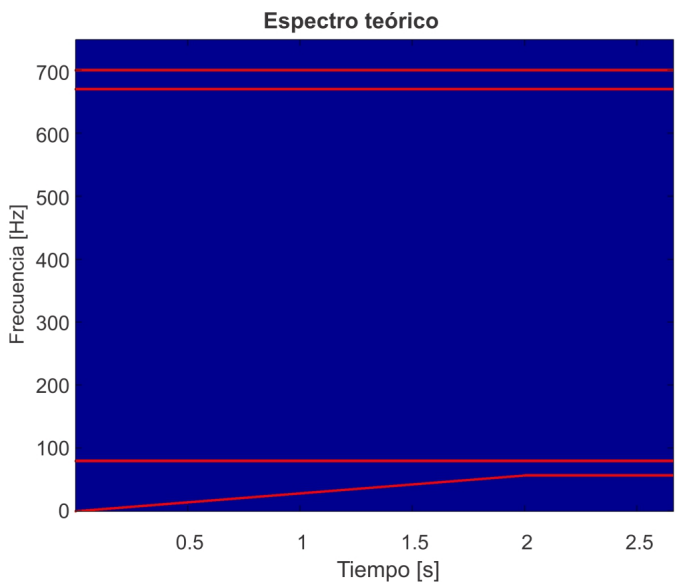

b)

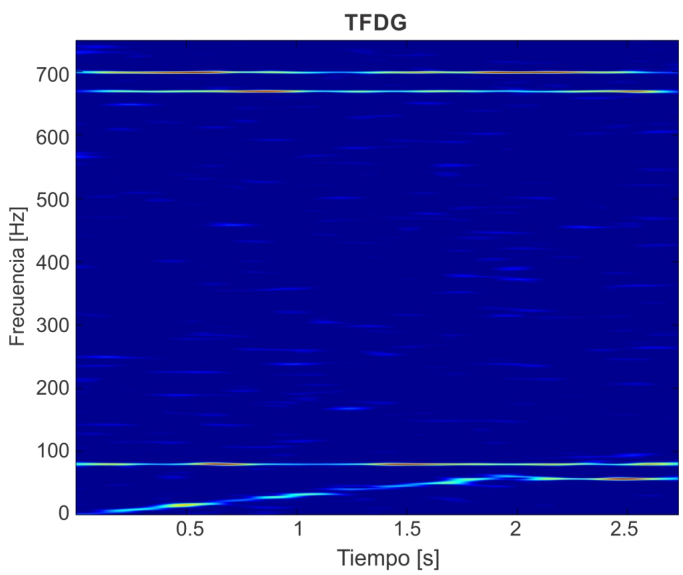

d)

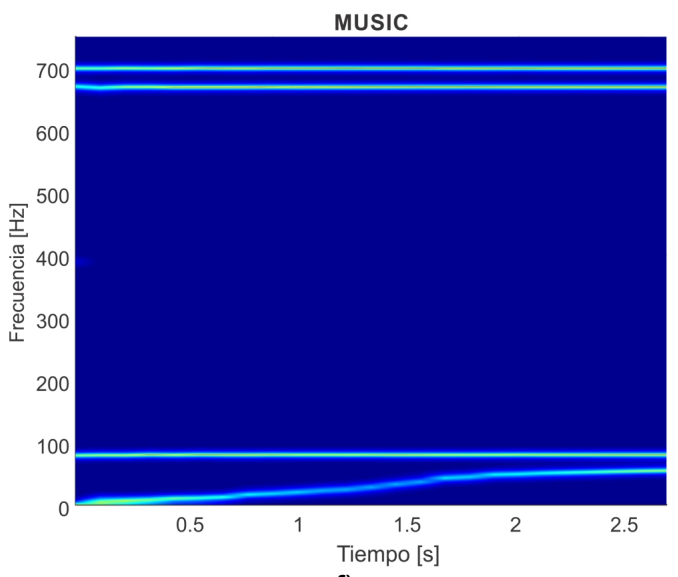

f)

Figura 4.8: Validación de las técnicas propuestas. 


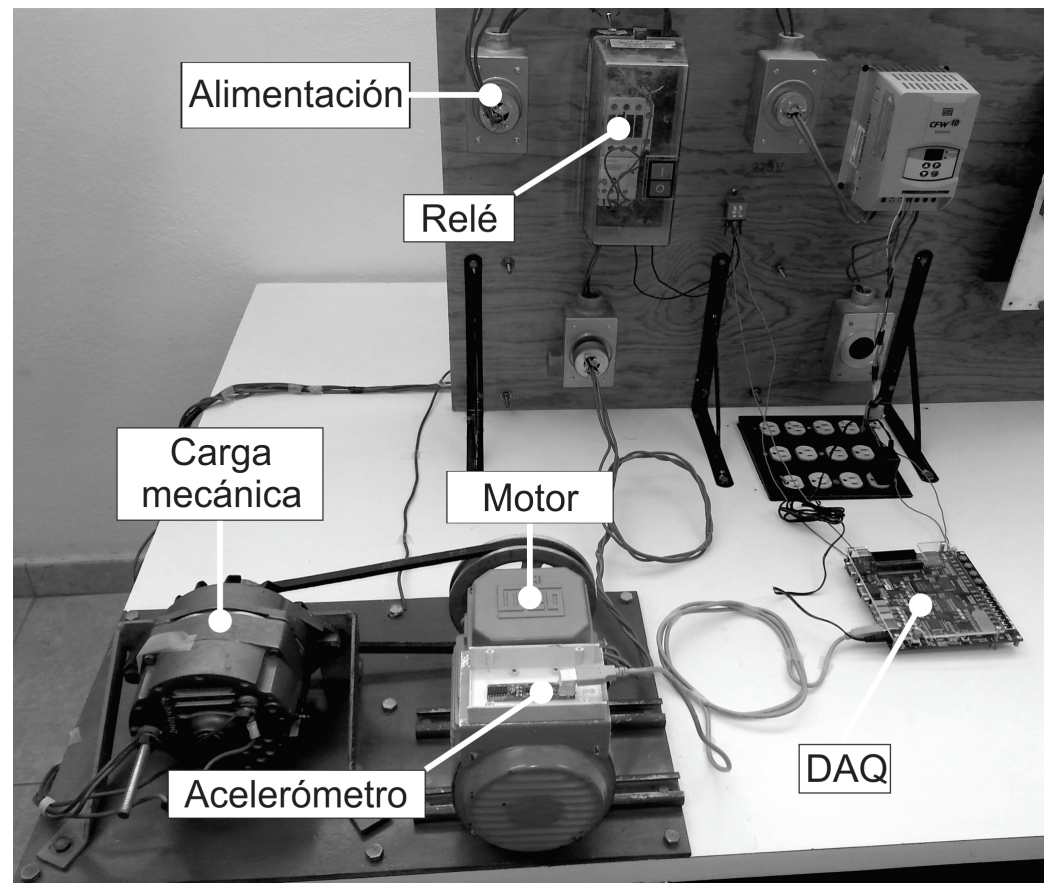

Figura 4.9: Banco de pruebas usado para los ensayos durante el transitorio de arranque.

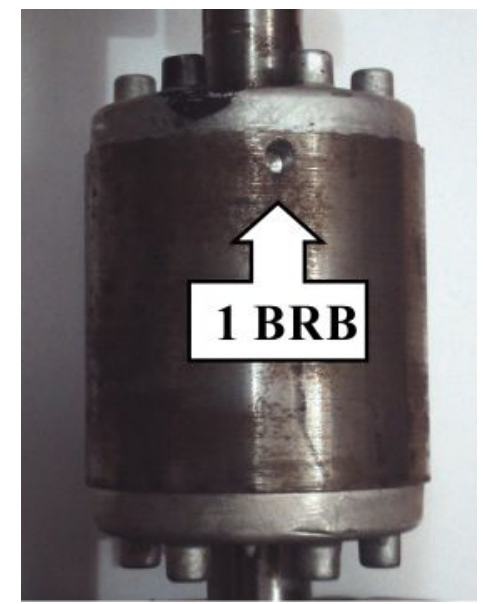

Figura 4.10: Rotor con una barra rota. 


\section{Capítulo 5}

\section{Resultados y discusión}

\subsection{Resultados del análisis en estado estable}

En esta sección, se presentan los resultados del análisis en estado estable para las señales de sonido y vibraciones. El sonido y las señales de vibración se analizan con la CEEMD y el marginal en frecuencia de la representación tiempo-frecuencia de Gabor. Estas señales fueron capturadas durante el funcionamiento en estado estacionario de los motores y bajo cuatro condiciones diferentes: sano, dos barras del rotor rotas, desbalance y defectos en el rodamiento.

Para el análisis en estado estable los parámetros que se utilizan para la CEEMD son: para la desviación estándar, $S D=0.2$, este valor se elige de acuerdo a lo propuesto por los autores del algoritmo, el número de realizaciones en las cuales la señal se contamina con ruido es de 300 y el límite de iteraciones para obtener las IMFs es de 500. Para el cálculo del marginal en frecuencia, la transformada de Gabor se calcula a través de $256(\mathrm{~N})$ coeficientes de Gabor de las 16384 muestras disponibles en la señal de sonido y de las 4096 muestras disponibles en la señal de vibraciones, además la ventana es del tipo Gaussiana de tamaño $N+1$.

Las IMFs fueron seleccionadas tras analizar las IMFs resultantes de la descomposición de las señales de sonido y vibración del motor sano y de los motores con fallas. Las IMFs que 
se seleccionan contienen las frecuencias relacionadas con la falla buscada o sus armónicos, es decir, contienen las frecuencias o armónicos asociados a la falla de las barras del rotor rotas, el desbalance y los defectos en el rodamiento. En el caso de la señal de sonido, las tres primeras IMFs contienen la información de la frecuencia deseada para el diagnóstico de las fallas, y en el caso de las señales de vibración, las cuatro primeras IMFs contienen la información deseada. Para cada falla, las IMFs se eligen de acuerdo a su contenido espectral. En algunos casos, se seleccionaron dos IMFs, debido a que la frecuencia de interés se encontraba en una región de frecuencia intermedia entre dos IMFs consecutivas. Para las vibraciones los mejores resultados se obtienen a partir de la señal de vibración en el eje $z$, Az, y esto se debe a que las vibraciones en un motor de inducción son típicamente vibraciones radiales (eje vertical o eje Az). Estas fuerzas radiales, que actúan sobre el estátor y el rotor, están asociadas con los flujos magnéticos que entran o salen de las superficies del entre-hierro en el motor de inducción y la presencia de componentes de flujo espectral producidos por las distribuciones de densidad de corriente y el ranurado del estátor y el rotor. La saturación magnética también introduce componentes adicionales no deseados en las fuerzas radiales [60]. Los resultados se presentan divididos en cuatro casos de estudio, y se realiza un análisis cualitativo de la condición de operación del motor.

\subsubsection{Caso de estudio: motor sano}

En la Figura 5.1, se muestran las tres primeras IMFs para la señal de sonido del motor sano. En la figura 5.2, se muestra el espectro de la señal de sonido, donde se pueden observar tres componentes de frecuencia mayor, uno localizado en el rango de 230-235 Hz. Este componente de frecuencia está asociado con el cuarto armónico de la frecuencia de rotación del rotor, que se sitúa a $231 \mathrm{~Hz}$. También hay otro componente de mayor magnitud situado a una frecuencia aproximada de $660 \mathrm{~Hz}$ y un tercer componente de frecuencia situado a $787 \mathrm{~Hz}$. La aplicación del CEEMD a esta señal da catorce IMFs. Sólo el espectro de las tres primeras IMFs se calcula a través del marginal en frecuencia de la TFDG porque tienen el contenido de frecuencia de interés y la mayor parte del contenido energético de la señal. 

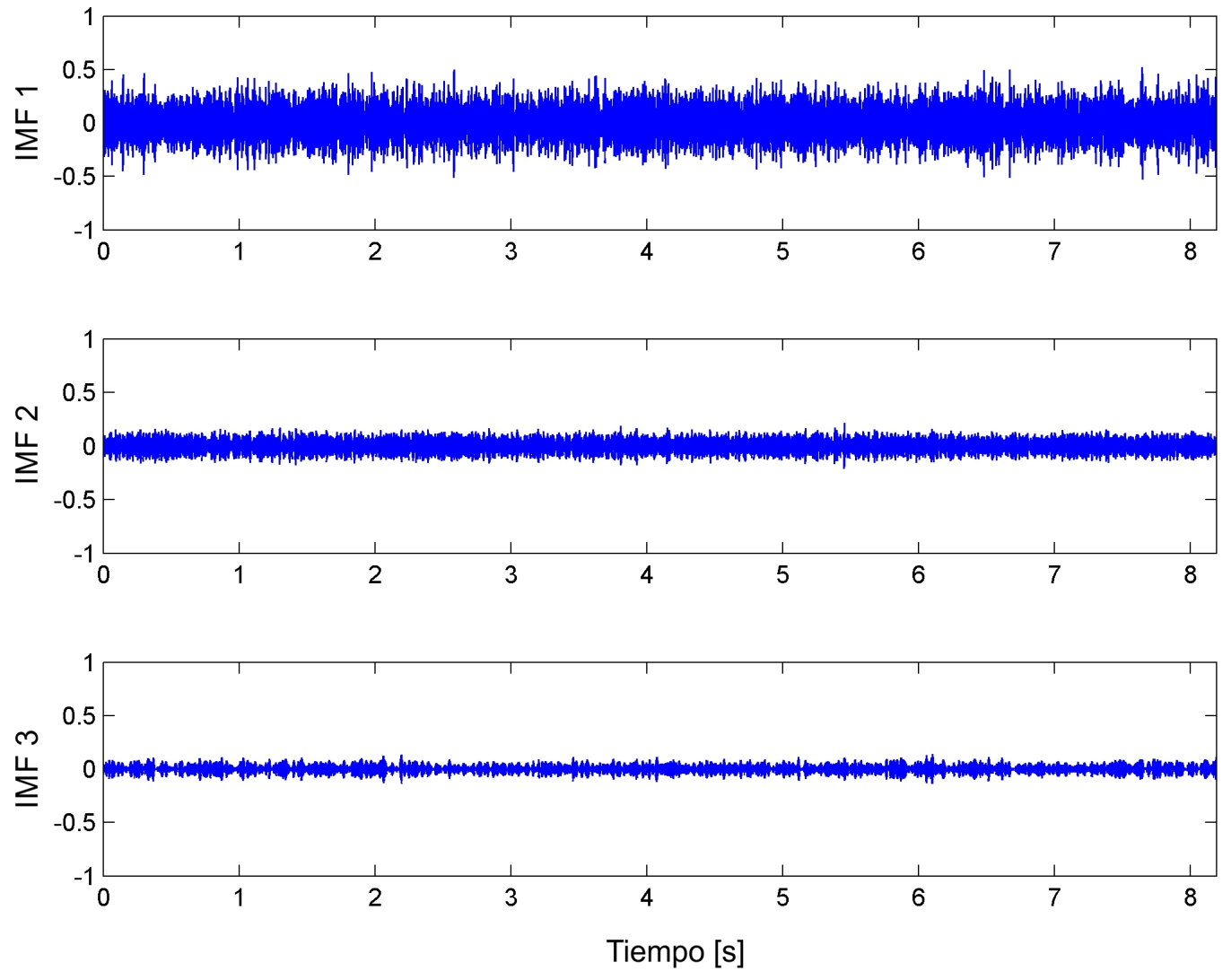

Figura 5.1: Primeras tres IMFs obtenidas de la señal de sonido para el motor sano.

La Figura 5.3 muestra las cuatro primeras descomposiciones de la señal de vibración del motor sano. El contenido espectral, mostrado en la Figura 5.4, tiene sólo un componente situado a $668 \mathrm{~Hz}$. Al aplicar la CEEMD a esta señal, su descomposición generó catorce IMFs, pero sólo las primeras cuatro se consideraron para obtener el espectro a través del cálculo del marginal en frecuencia de la TFDG. El estudio del motor sano no produjo otros componentes de frecuencia significativa, y estas representaciones (Figuras 5.3 y 5.4) serán la referencia para comparar con las condiciones con falla del motor. 


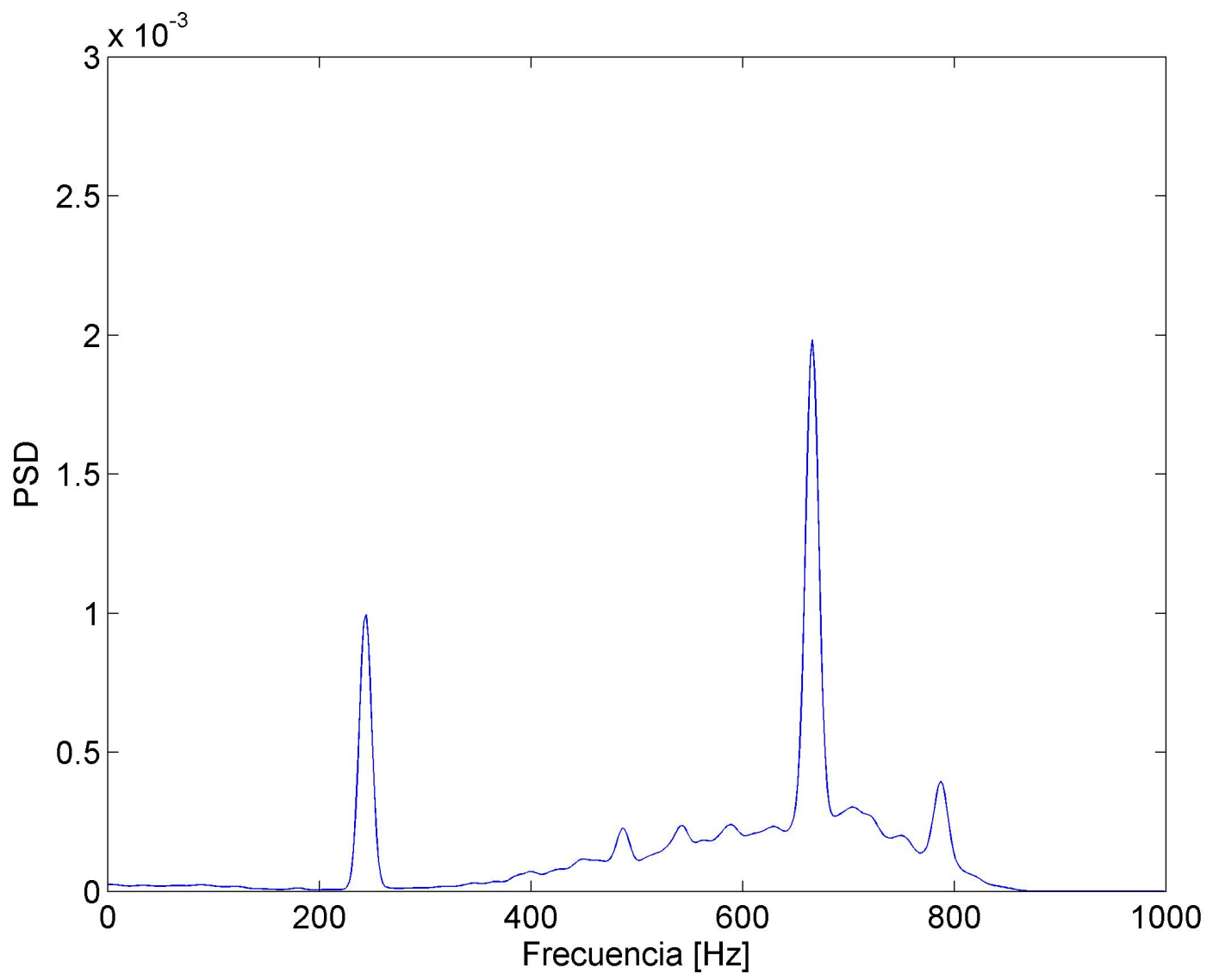

Figura 5.2: Espectro de las primeras tres IMFs obtenidas de la señal de sonido para el motor sano.

\subsubsection{Caso de estudio: Dos barras del rotor rotas (2BRB)}

Los valores de frecuencia que se calculan a partir de la ecuación (2.7) podrán aparecer o no en el espectro de vibraciones y sonido. En este caso, para las señales analizadas los valores de frecuencia que se pueden identificar en el espectro de sonido son los que se muestran en la tabla 5.1 .

La Figura 5.5 muestra las tres primeras IMFs de la señal de sonido de la falla 2BRB. El espectro se calcula considerando la primera IMF solamente, que es la que contiene la infor- 

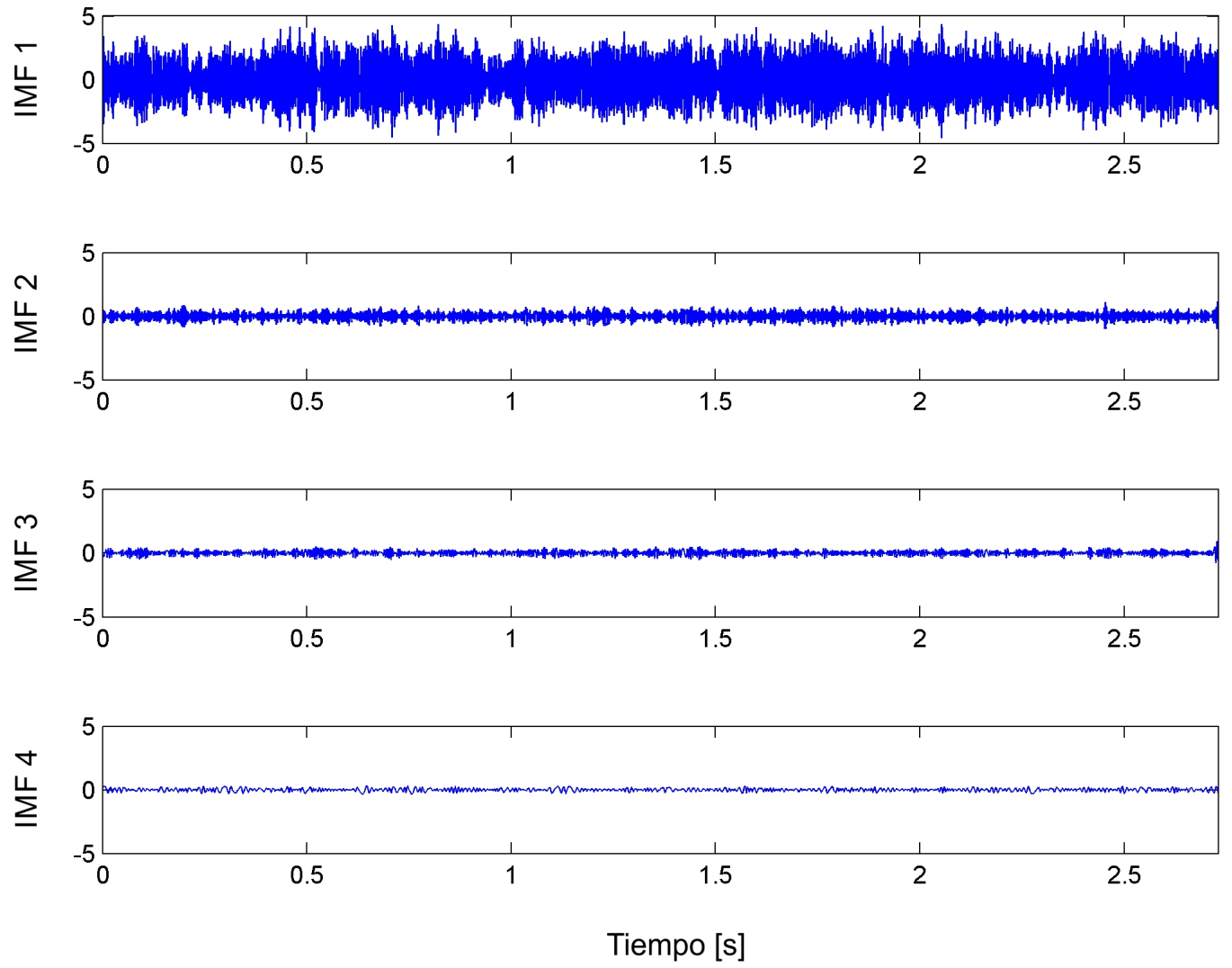

Figura 5.3: Primeras cuatro IMFs obtenidas de la señal de vibraciones para el motor sano.

mación relacionada con esta falla. La Figura 5.6 (b) representa el espectro obtenido, donde hay varios componentes espectrales que no están presentes en el espectro cuando el motor se encuentra sano (Figura 5.6 (a)). El primer componente se encuentra aproximadamente a $448.5 \mathrm{~Hz}\left(f_{S 1}\right.$ en la figura), correspondiente al octavo armónico de $f r$. También se observan otros dos pares de componentes situados aproximadamente a $582 \mathrm{~Hz}\left(f_{S 2}\right.$ en la figura) y 612.5 $H z$ ( $f_{S 3}$ en la figura), correspondientes al décimo armónico de $f r$ y al undécimo armónico de $f r$, respectivamente. Finalmente, se observa otro conjunto de armónicos con una amplitud mayor que el conjunto anterior. Estos se encuentran a $711 \mathrm{~Hz}\left(f_{S 4}\right.$ en la figura) y $728.2 \mathrm{~Hz}$ 


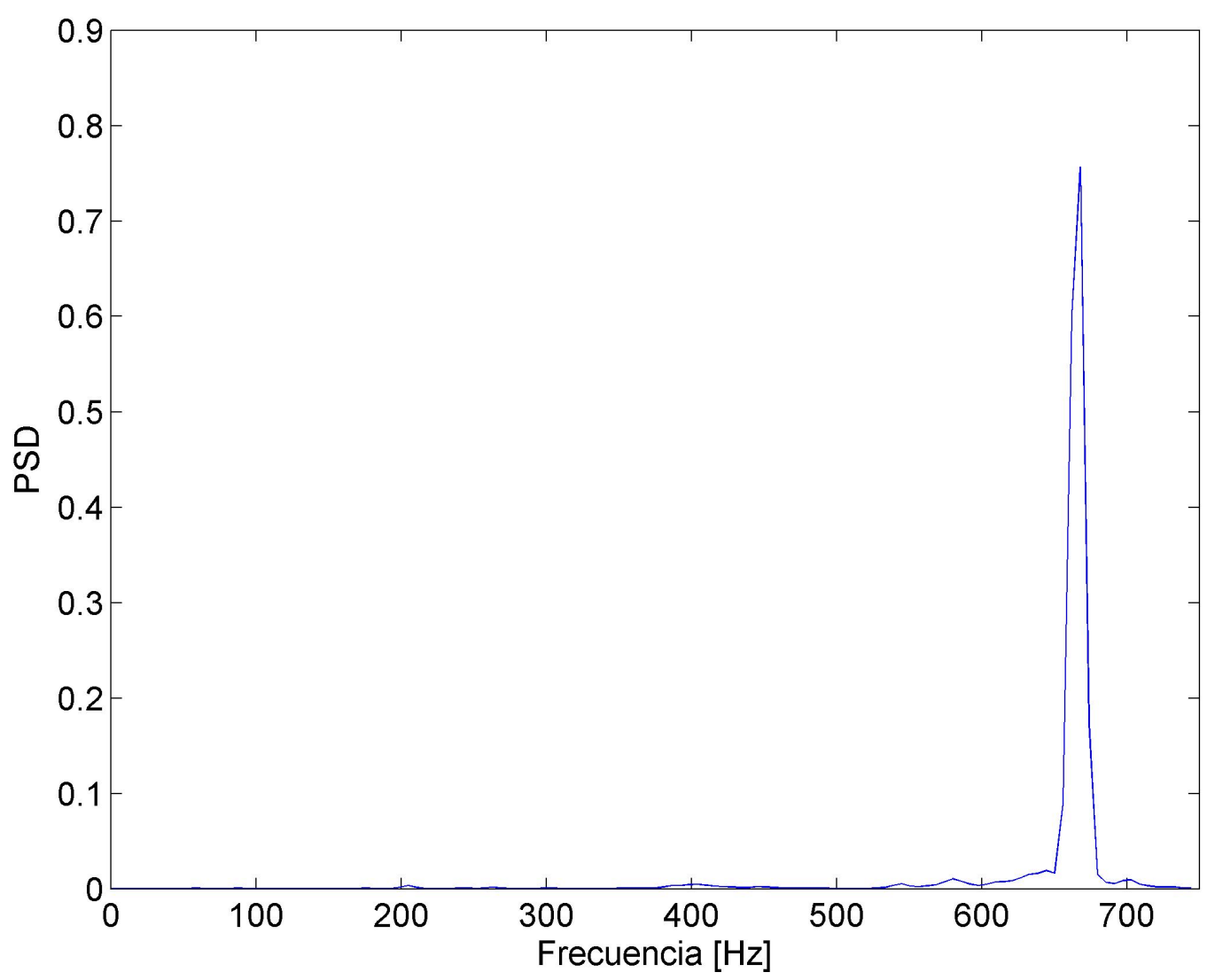

Figura 5.4: Espectro de las primeras tres IMFs obtenidas de la señal de vibraciones para el motor sano.

( $f_{S 5}$ en la figura) y corresponden al duodécimo armónico de $f r$ y al decimotercero armónico de $f r$, respectivamente.

En el espectro de vibraciones se pueden identificar los componentes mostrados en la tabla 5.2. La Figura 5.7 muestra las cuatro primeras IMFs de la señal de vibración para la falla 2BRB y la Figura 5.8 el espectro correspondiente de la primera IMF. En la Figura 5.8 (b), se pueden observar tres componentes espectrales asociados con la falla 2BRB. La primera es 
Tabla 5.1: Valores de frecuencias de falla detectadas en la señal de sonido para 2BRB.

\begin{tabular}{|c|c|c|}
\hline Armónico de $f r$ & Valor de $n$ en la ecuación 2.7 & Frecuencia asociada a la falla (Hz) \\
\hline \hline 8 & 3 & 448.5 \\
\hline 10 & 1 & 582 \\
\hline 11 & 5 & 612.5 \\
\hline 12 & 4 & 711 \\
\hline 13 & 5 & 728.2 \\
\hline
\end{tabular}

la frecuencia de $484.5 \mathrm{~Hz}\left(f_{V 1}\right.$ en la figura), que no está presente en la señal del motor sano (Figura 5.8 (a)). El segundo componente espectral, situado a $586.5 \mathrm{~Hz}$ ( $f_{V 2}$ en la figura), tiene la mayor amplitud de energía. Finalmente, hay otro componente a la frecuencia de 728.2 $H z$ ( $f_{V 3}$ en la figura). El componente situado a $668 \mathrm{~Hz}$ es el único presente en la señal de vibración para el motor sano (Figura 5.8 (a)).

Tabla 5.2: Valores de frecuencias de falla detectadas en la señal de vibraciones para 2BRB.

\begin{tabular}{|c|c|c|}
\hline Armónico de $f r$ & Valor de $n$ en la ecuación $[2.7$ & Frecuencia asociada a la falla $(\mathrm{Hz})$ \\
\hline \hline 8 & 5 & 484.5 \\
\hline 10 & 2 & 586.5 \\
\hline 13 & 5 & 728.2 \\
\hline
\end{tabular}

\subsubsection{Caso de estudio: Desbalance (UNB)}

La Figura 5.9 muestra las tres primeras IMFs proporcionadas por el análisis CEEMD de la señal de sonido. En este caso de estudio, sólo se consideran las dos primeras IMFs, ya que son las que contienen información relevante para esta falla. La frecuencia fundamental de esta falla es la frecuencia de rotación del rotor, que en este caso es $57.75 \mathrm{~Hz}$, y en la tabla 5.3 se muestran las frecuencias asociadas a esta falla que se pueden identificar en el espectro de sonido. La Figura 5.10 muestra el espectro obtenido calculando el marginal en frecuencia de la TFDG de las dos primeras IMFs. En la figura 5.10 (b) se pueden ver claramente cuatro 

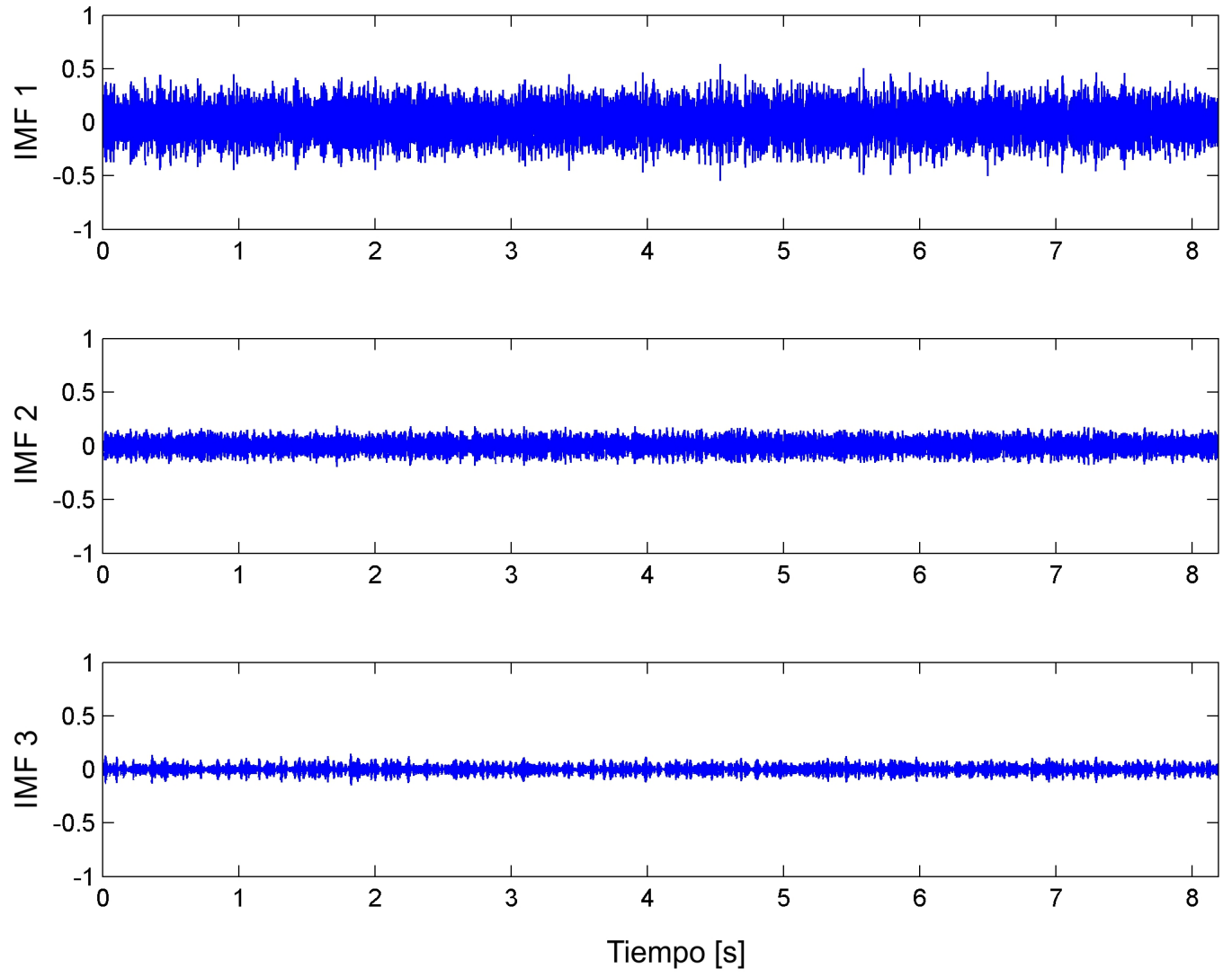

Figura 5.5: Primeras tres IMFs de la señal de sonido para el motor con dos barras rotas.

componentes espectrales de frecuencia en la región de interés. El primer componente es a $340 \mathrm{~Hz}$ correspondiente al sexto armónico de la frecuencia de rotación del rotor. El segundo componente, situado a $395 \mathrm{~Hz}$, corresponde al séptimo armónico. El tercer componente tiene una amplitud menor que los componentes anteriores, y está situado a $450 \mathrm{~Hz}$. Esta frecuencia corresponde al octavo armónico. Finalmente, se puede identificar el noveno armónico localizado a $510 \mathrm{~Hz}$.

La Figura 5.11 representa las cuatro primeras IMFs obtenidas con la CEEMD a partir 
Tabla 5.3: Valores de frecuencias de falla detectadas en la señal de sonido para el desbalance.

\begin{tabular}{|c|c|}
\hline Armónico de $f r$ & Frecuencia asociada a la falla (Hz) \\
\hline \hline 6 & 346.5 \\
\hline 7 & 404.2 \\
\hline 8 & 462 \\
\hline 9 & 519.7 \\
\hline
\end{tabular}

de la señal de vibración del motor con desbalance. La cuarta IMF se utiliza para calcular el espectro de la señal de vibración porque es el único que contiene la información de interés, en la señal de vibraciones el único componente relacionado con esta falla que se identificó fue el de la frecuencia de rotación. En la Figura 5.12 (b), se muestra un componente a $57.75 \mathrm{~Hz}$, que no está presente en el espectro de la señal de vibración para el motor sano (Figura 5.12 (a)), revelando claramente la presencia del desbalance.

\subsubsection{Caso de estudio: Defectos en el rodamiento (BDF)}

Las primeras tres IMFs, obtenidas con la CEEMD aplicada a la señal de sonido, se muestran en la Figura 5.13. En este caso, la información relevante sobre la falla está en las dos primeras descomposiciones. Como se ha mencionado anteriormente, un defecto en la pista exterior del rodamiento provoca un impulso cada vez que un elemento rodante contacta con el defecto. Así, la frecuencia asociada a esta falla está asociada con la rotación del eje y la frecuencia de falla $\left(f_{B P O F}\right)$, se sitúa a $177.7 \mathrm{~Hz}$. En la tabla 5.4 se muestran los valores de las frecuencias asociadas a la falla BDF detectados en la señal de sonido, además para la señal de vibraciones, las frecuencias asociadas al tercero y cuarto armónico también aparecen en el espectro. Para este caso de estudio, el espectro obtenido de la señal de sonido se muestra en la Figura 5.14. En esta figura, se ven tres armónicos asociados con la falla: el tercer armónico localizado a $533.1 \mathrm{~Hz}$; el cuarto armónico situado a $710.8 \mathrm{~Hz}$ y otro armónico con menor amplitud situado a $888.5 \mathrm{~Hz}$, asociado al quinto armónico. Estos tres componentes espectrales no se observan en el espectro de la señal de sonido del motor sano (Figura 5.14 
(b)), demostrando la presencia de la falla.

Tabla 5.4: Valores de frecuencias de falla detectadas en la señal de sonido para BDF.

\begin{tabular}{|c|c|}
\hline Armónico de $f r$ & Frecuencia asociada a la falla $(\mathrm{Hz})$ \\
\hline \hline 3 & 533.1 \\
\hline 4 & 710.8 \\
\hline 5 & 888.5 \\
\hline
\end{tabular}

En la Figura 5.15, se muestran las cuatro primeras IMFs, obtenidas de la aplicación de la CEEMD a la señal de vibración de un motor con defectos en el rodamiento. En este caso, la información de interés está en la primera IMF. En la Figura 5.16 (a), se muestra el espectro de la primera IMF de la señal de vibración del motor sano y en la Figura 5.16 (b), se muestra el espectro de la primera IMF para el motor con defectos en el rodamiento. En este último se observan dos componentes espectrales asociados a esta falla: uno correspondiente al tercer armónico de $f_{B P O F}$ y otro correspondiente a su cuarto armónico. Comparando con la Figura 5.16 (a), se observa que estos componentes no están presentes en el espectro de las IMFs del motor sano.

Como conclusión de todos los casos estudiados, el análisis de señales sonoras mediante el uso de la CEEMD en combinación con el marginal en frecuencia de la TFDG es una metodología viable para la detección de fallas en motores de inducción. Esto ha sido corroborado por el análisis de las señales de vibración. La CEEMD facilitó la detección de las fallas eliminando componentes espectrales indeseables presentes en la señal. En la mayoría de los casos estudiados, se localizaron varios componentes espectrales asociados con la falla. La falla de las barras del rotor rotas produce una firma más débil en el espectro de la señal de sonido que en el espectro de la señal de vibración. Esto se debe probablemente a su menor contribución a la generación de vibraciones mecánicas, que también afecta a la producción de sonido. En las otras fallas analizadas (UNB y BDF), las frecuencias relacionadas con la falla presentaron un mayor contenido energético, haciéndolas más detectables, y esto se debe a que se manifiestan principalmente como una perturbación mecánica, lo que provoca un au- 
mento de vibraciones y, por lo tanto, un aumento en el nivel de sonido emitido por el motor. La Tabla 1 muestra una comparación de detectabilidad entre la técnica propuesta en este trabajo y las técnicas Wigner-Ville y MUSIC. Los resultados para las técnicas Wigner-Ville y MUSIC fueron presentados en [37]. La detectabilidad se calcula como la relación de amplitud (en dB) entre la frecuencia asociada a la falla sobre la frecuencia en el espectro del motor sano.

Se ha demostrado que la metodología propuesta para analizar las señales de sonido y vibración de los motores de inducción durante el funcionamiento en estado estacionario es claramente mejor que la FFT. La combinación de la CEEMD y el marginal en frecuencia de la TFDG ofrece una ventaja en señales con ruido contra la técnica clásica de análisis espectral, es decir, la FFT. La CEEMD elimina componentes de señal no deseados, y el marginal en frecuencia de la TFDG muestra tolerancia al ruido y proporciona suavizado del espectro resultante.

Tabla 5.5: Detectabilidad en dB para las fallas analizadas

\begin{tabular}{|l|c|c|c|c|}
\hline Condición del motor & Marginal en frecuencia de la TFDG & FFT & Wigner-Ville & MUSIC \\
\hline \hline 2BRB & 28 & 1 & 5 & 20 \\
\hline UNB & 37 & 0 & - & 30 \\
\hline BDF & 39 & 8 & 15 & 20 \\
\hline
\end{tabular}

FFT: Fast Fourier Transform, MUSIC: Multiple Signal Classification. 

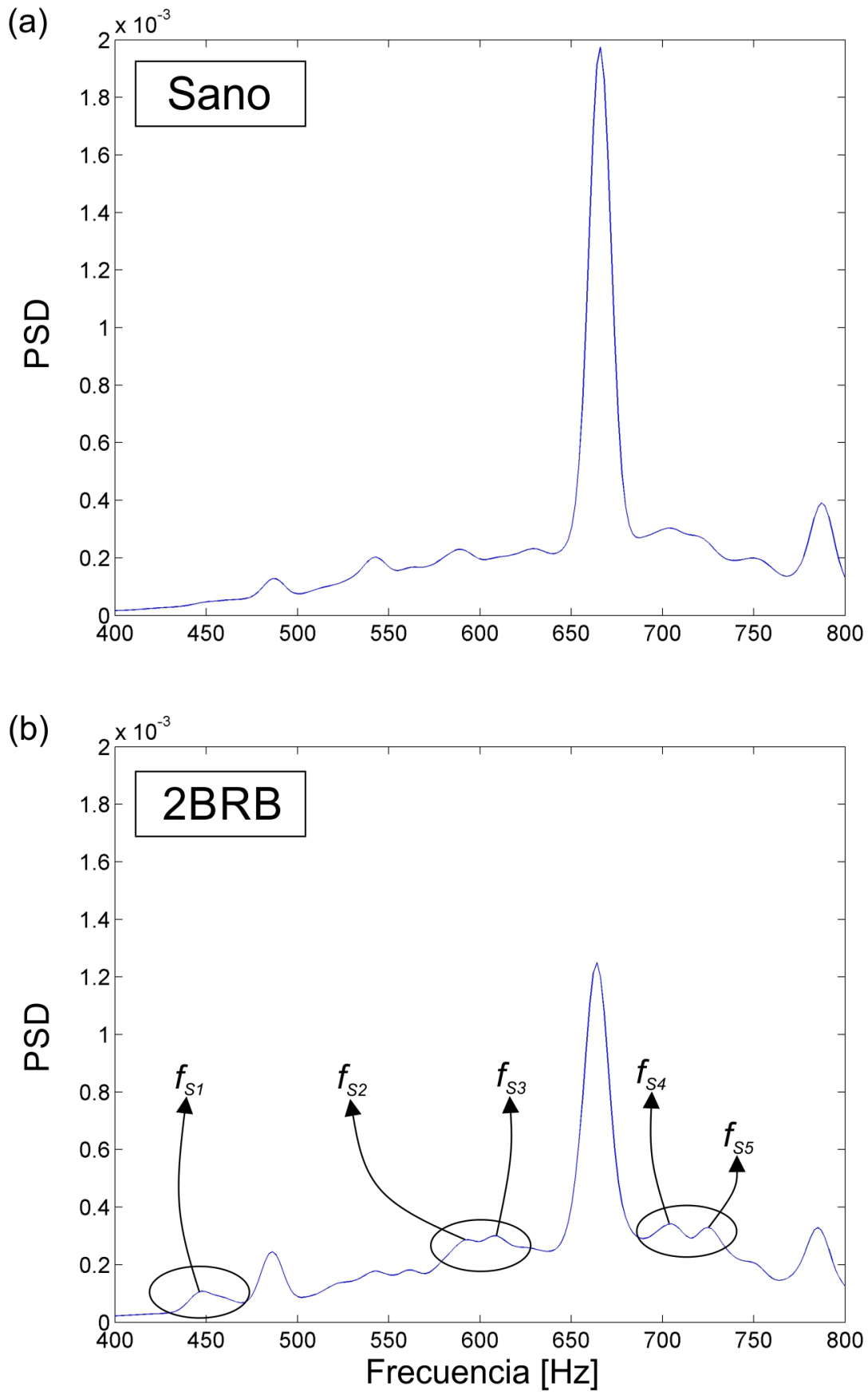

Figura 5.6: Espectro de la señal de sonido para a) el motor sano y b) el motor con dos barras rotas. 

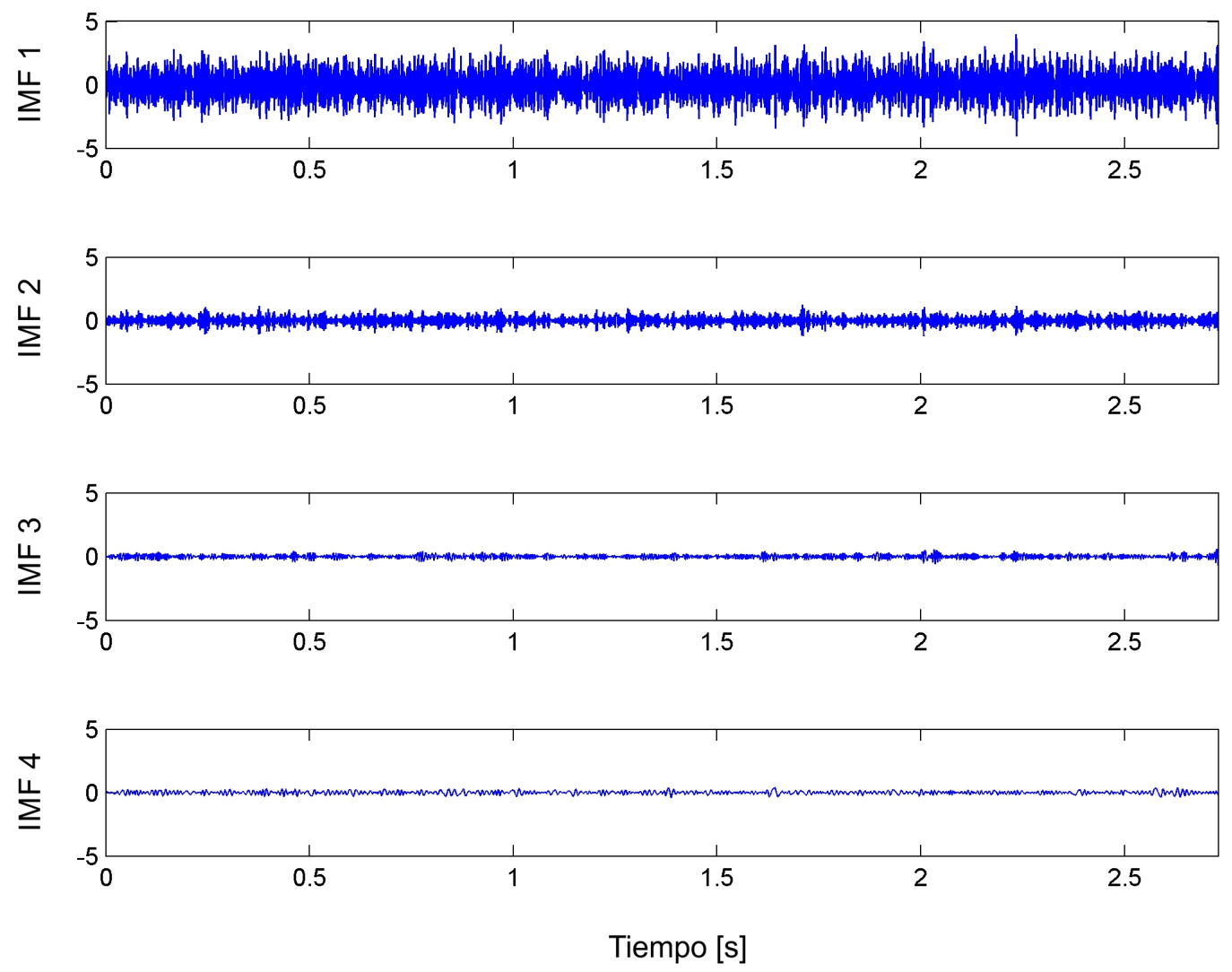

Figura 5.7: Primeras cuatro IMFs de la señal de vibracines para el motor con dos barras rotas. 
(a)

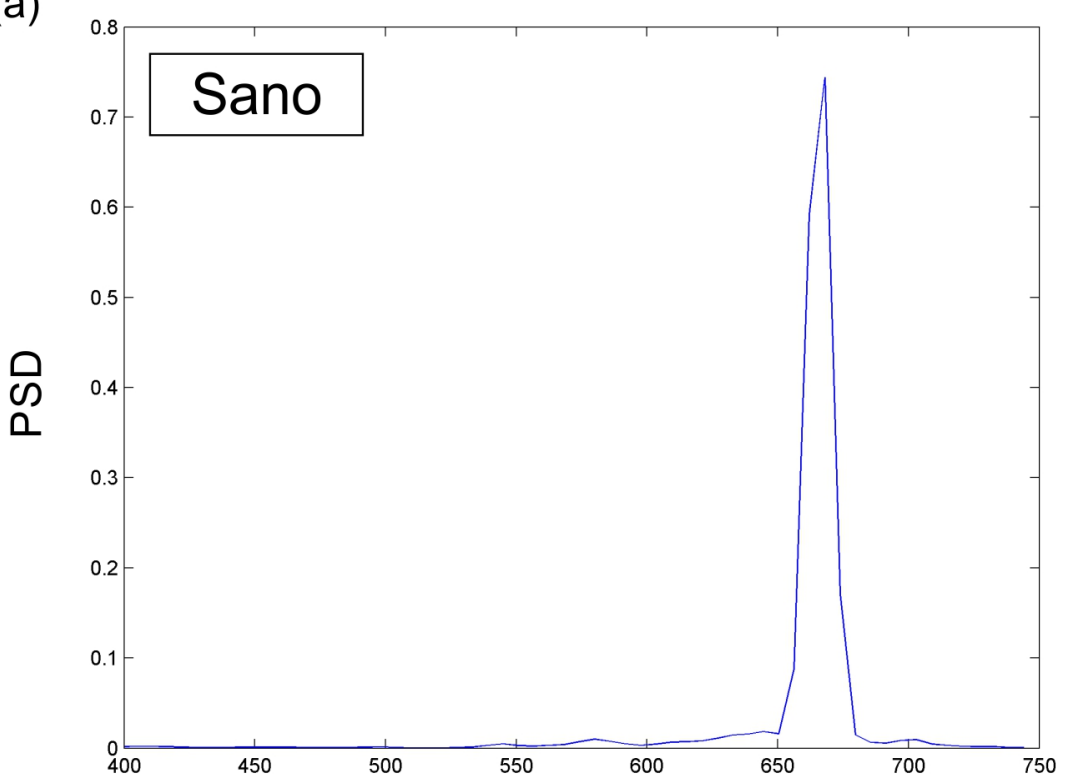

(b)

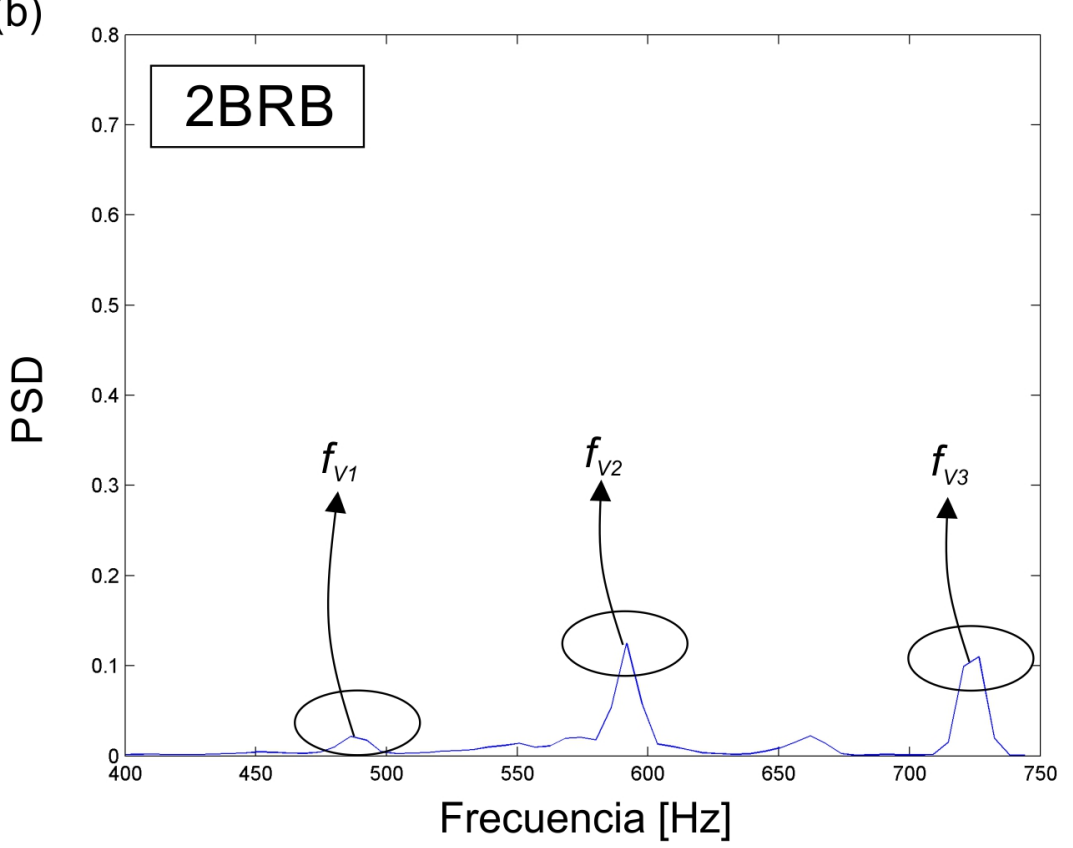

Figura 5.8: Espectro de la señal de vibraciones para a) el motor sano y b) el motor con dos barras rotas. 

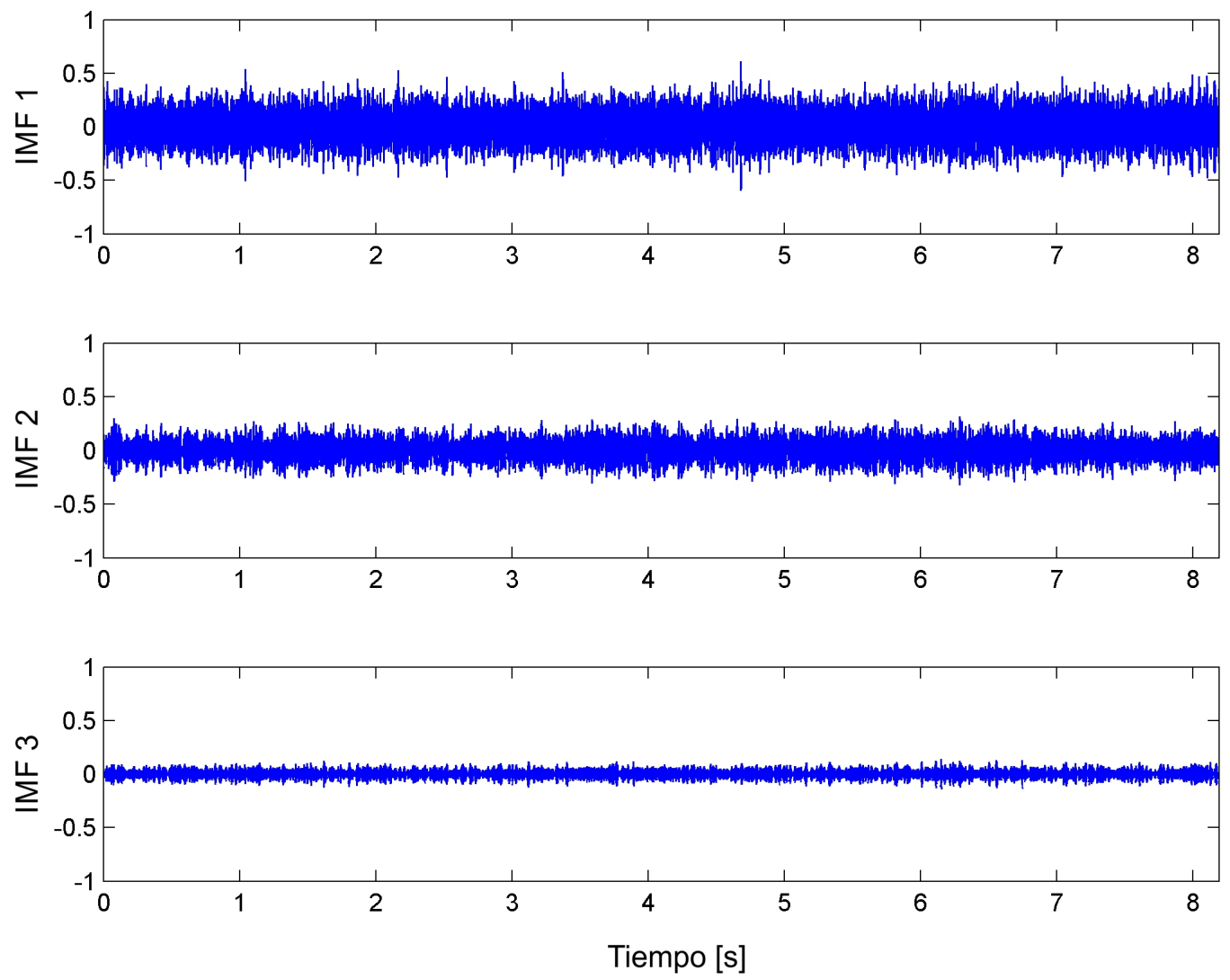

Figura 5.9: Primeras tres IMFs de la señal de sonido para el motor con desbalance. 

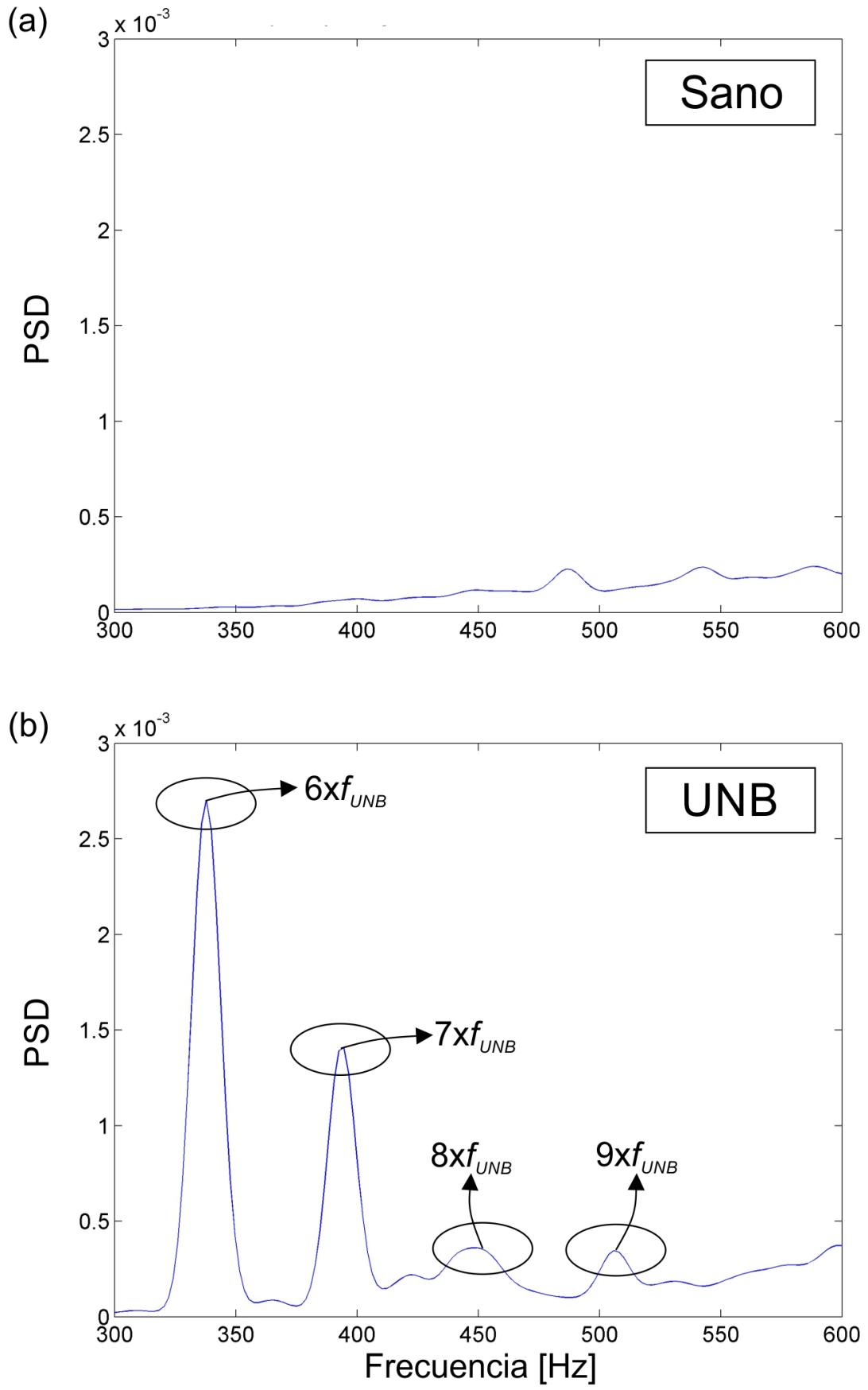

Figura 5.10: Espectro de la señal de sonido para a) el motor sano y b) el motor con desbalance. 

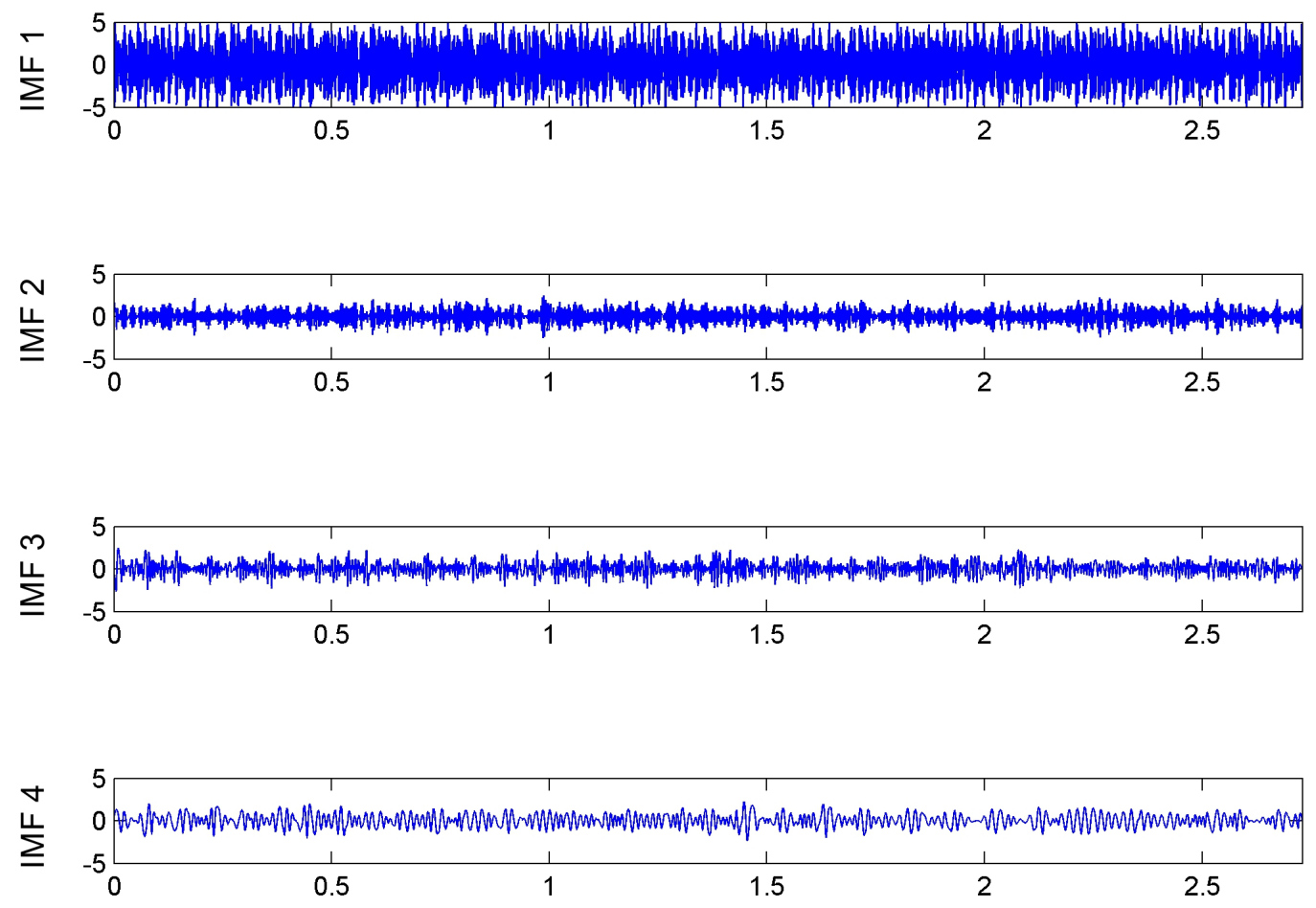

Tiempo [s]

Figura 5.11: Primeras cuatro IMFs de la señal de vibracines para el motor con desbalance. 
(a)

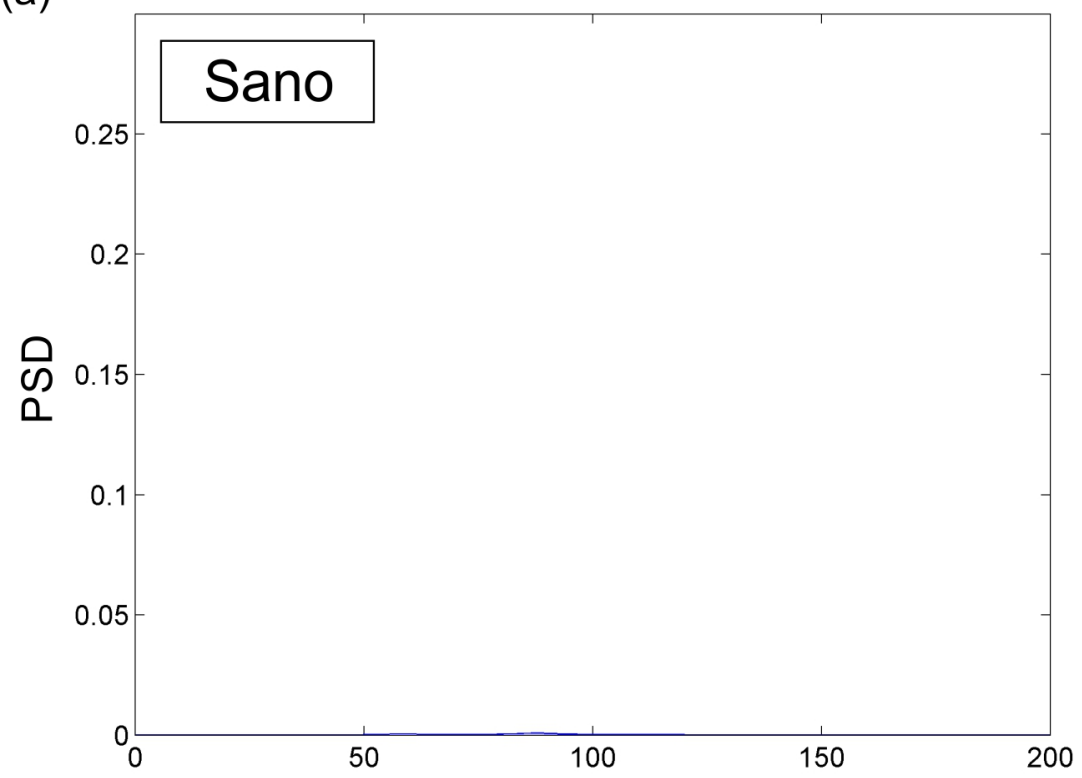

(b)

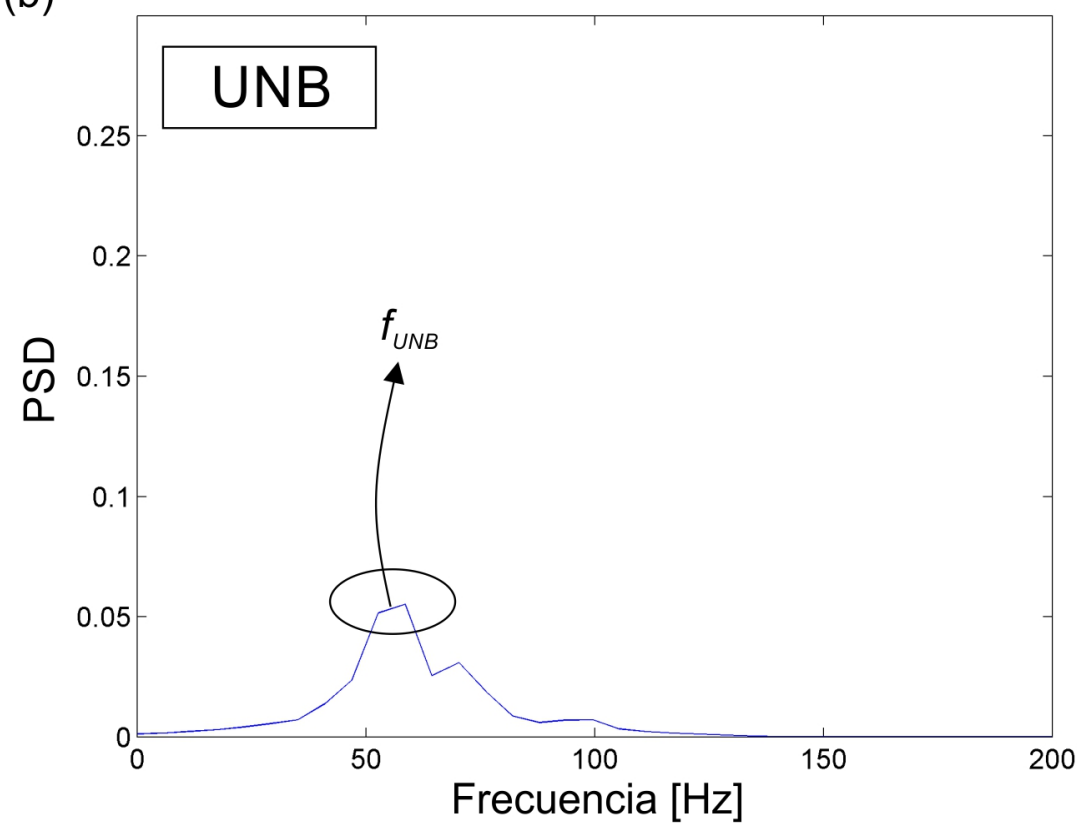

Figura 5.12: Espectro de la señal de vibraciones para a) el motor sano y b) el motor con desbalance. 

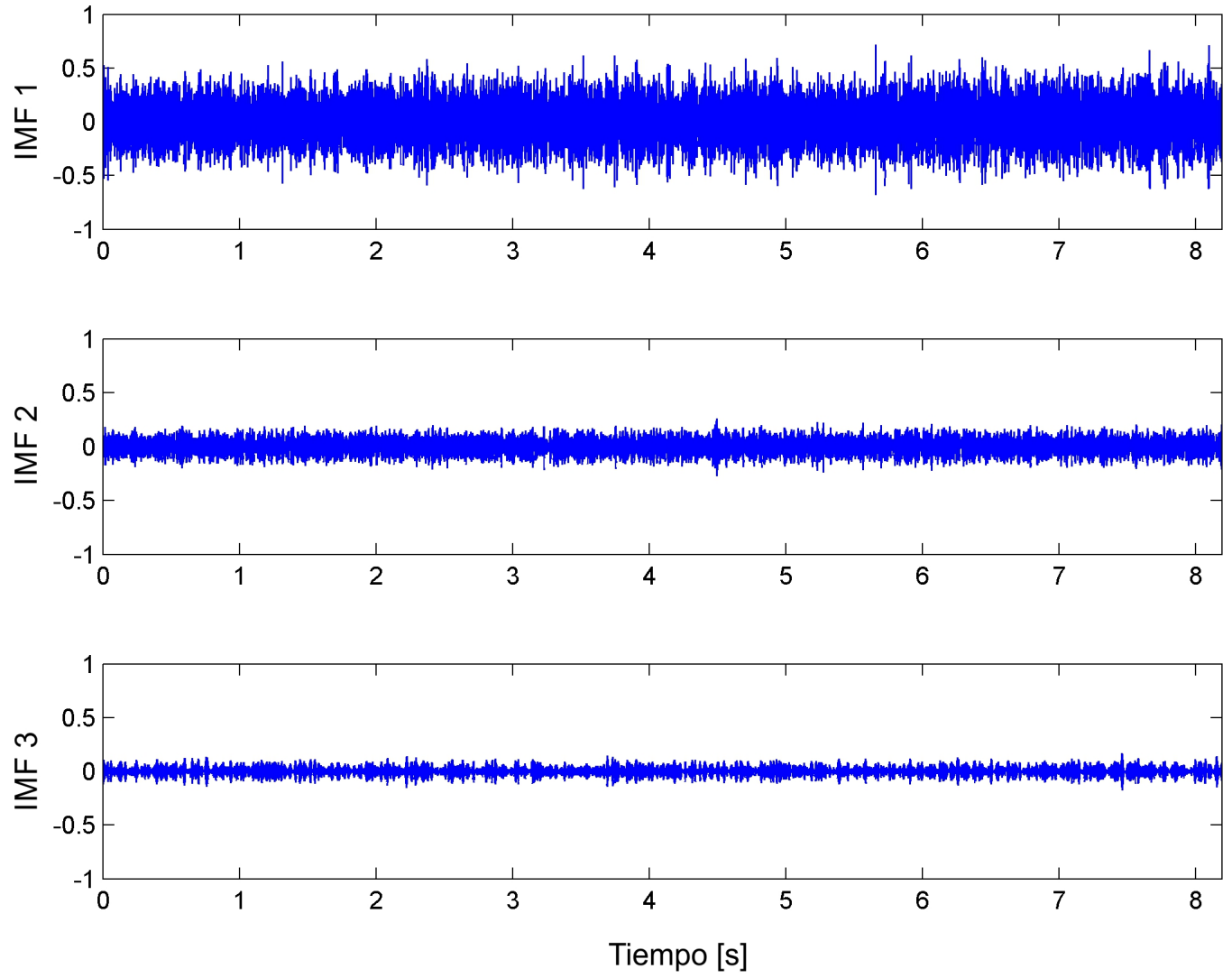

Figura 5.13: Primeras tres IMFs de la señal de sonido para el motor con defectos en el rodamiento. 
(a)

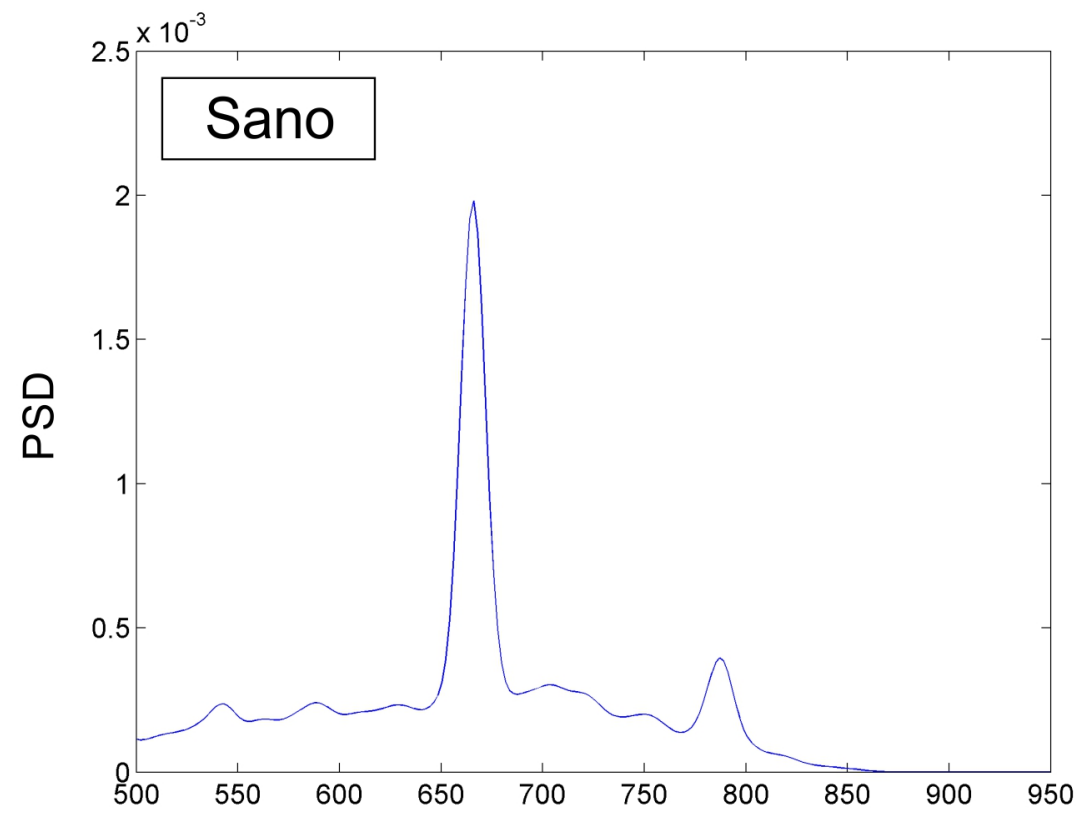

(b) $2.5 \times 10$

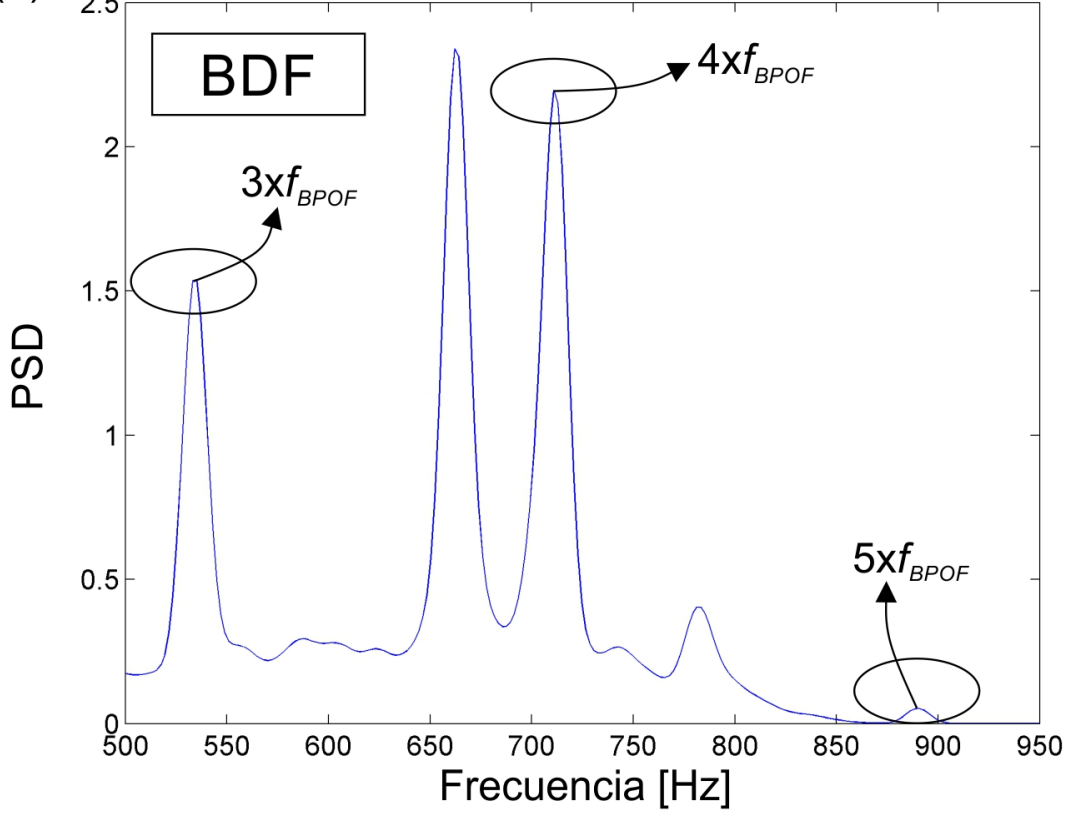

Figura 5.14: Espectro de la señal de sonido para a) el motor sano y b) el motor con defectos en el rodamiento. 

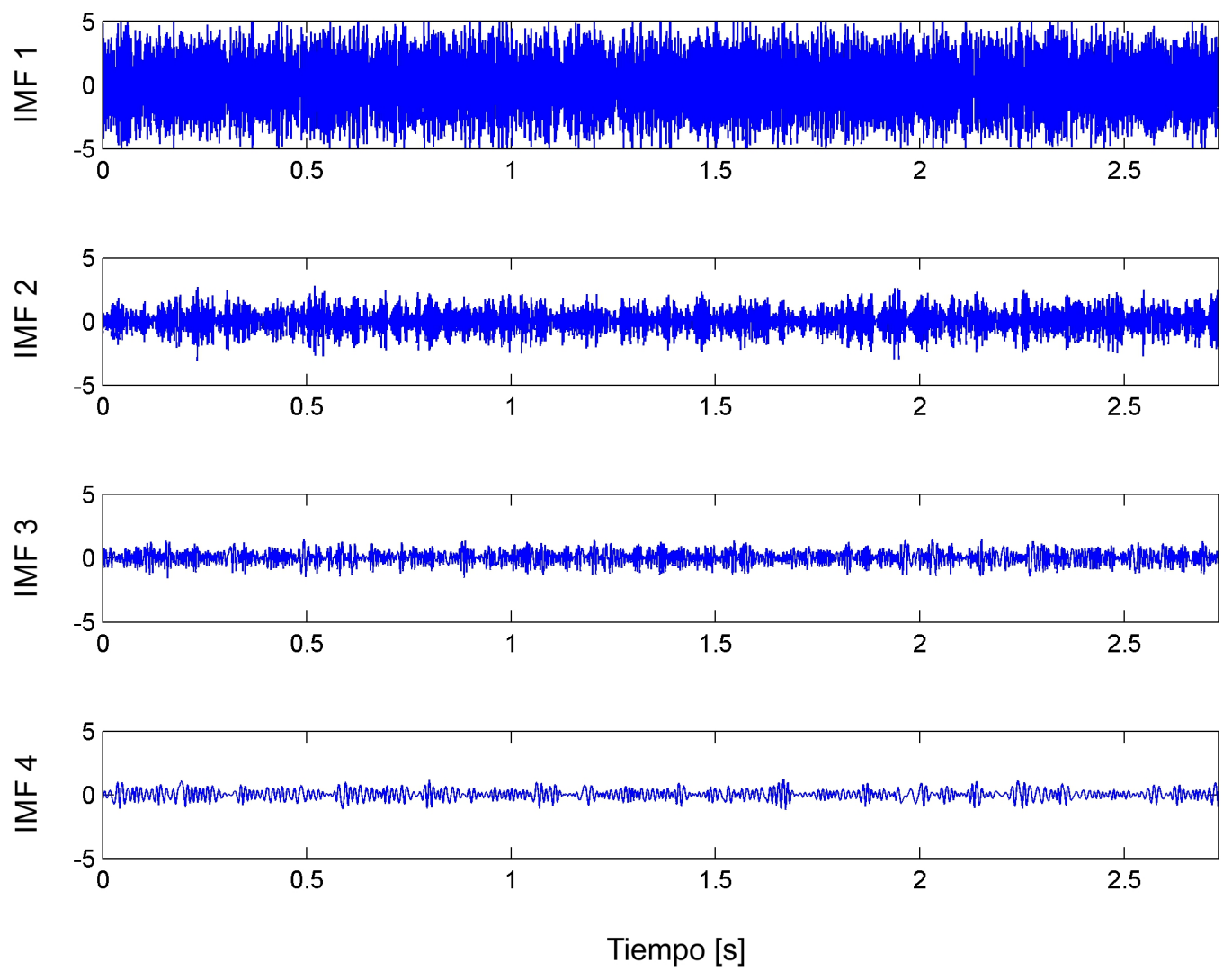

Figura 5.15: Primeras cuatro IMFs de la señal de vibracines para el motor con defectos en el rodamiento. 
(a)

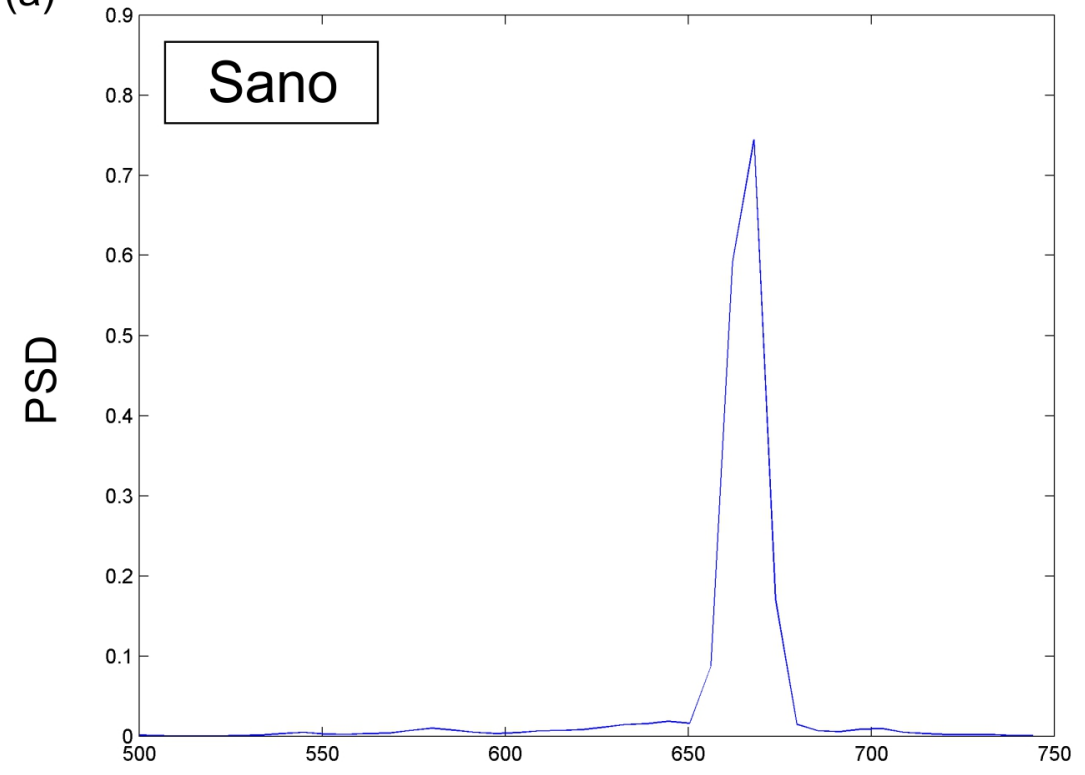

(b)

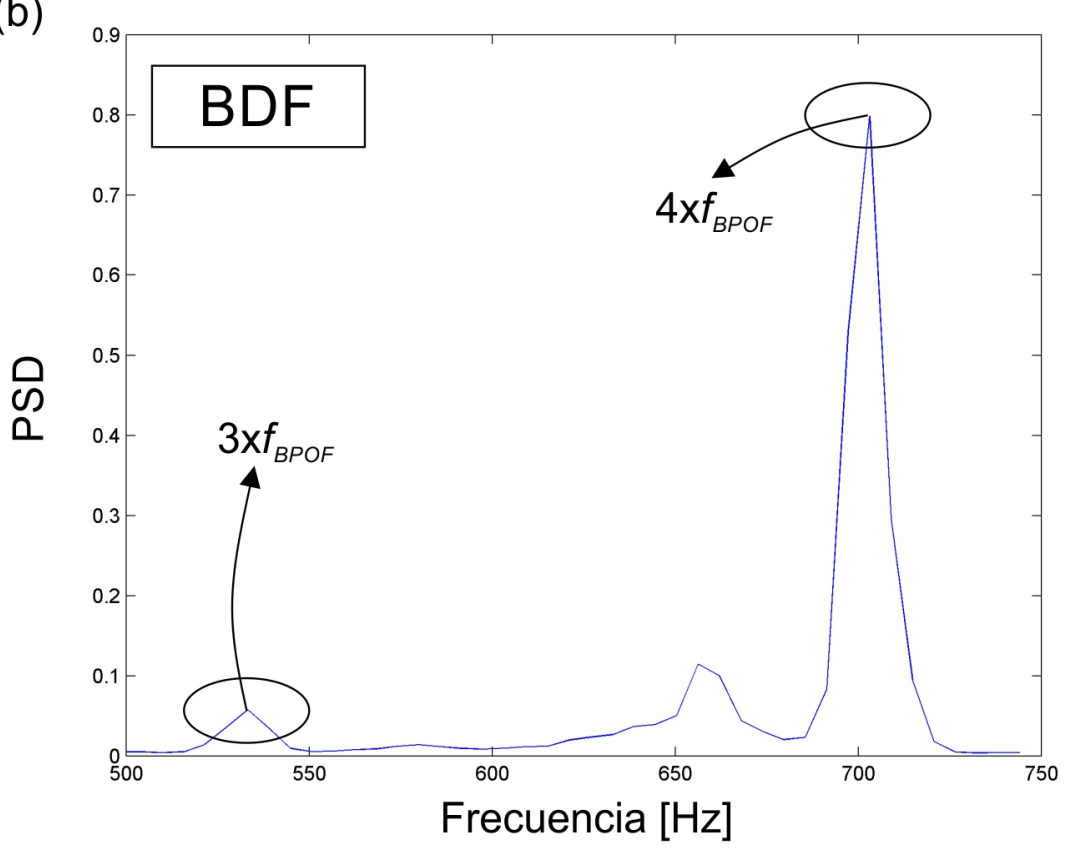

Figura 5.16: Espectro de la señal de vibraciones para a) el motor sano y b) el motor con defectos en el rodamiento. 


\subsection{Resultados del análisis en el transitorio de arran- que}

En esta sección se presentan los resultados obtenidos después de analizar las señales de vibración con las técnicas de descomposición de tiempo-frecuencia TFDG, TFMS y MUSIC, incluyendo una comparación con la STFT. Para la implementación de las técnicas durante el transitorio de arranque se consideraron los siguientes parámetros para las representaciones tiempo-frecuencia. Para la STFT la ventana empleada es del tipo Hamming con un tamaño de 128 muestras, también se usan 128 muestras para el cálculo de la FFT y se usa un traslape de 64 muestras. Para la transformada de Gabor se usaron un número de coeficientes igual a $2048(\mathrm{~N})$ y el ancho de la ventana Gaussiana es igual a $N+1$. Para MUSIC se emplearon secciones de la señal de 500 muestras, con ventanas de 150 muestras y traslape de 100 muestras entre las ventanas, además el orden utilizado en MUSIC es igual a 10. Para el escalograma de Morlet, la wavelet madre es la de Morlet, el producto tiempo-ancho de banda es igual a 2.5 y se emplea el total de las muestras de la señal para el cálculo de la representación.

Las ventanas que se usaron en el análisis con la STFT y la TFDG, son la ventana Hamming y la ventana gaussiana, respectivamente. La figura 5.17 muestra el espectrograma de una ventana Hamming de 512 puntos multiplicada por una señal sinusoidal de $32 \mathrm{~Hz}$. La figura 5.18 muestra el espectrograma de una ventana gaussiana de 512 puntos y desviación estándar, $S D=64$, y la figura 5.19 muestra el espectrograma de una ventana gaussiana de 512 puntos y desviación estándar, $S D=4$. Como podemos observar en la descomposición tiempo-frecuencia de la ventana Hamming (figura) y de la ventana gaussiana con $S D=64$ (figura), sus espectros son muy similares, sin embargo, la ventana gaussiana con $S D=4$ hace que se concentre la energía en un punto en el espectro. La longitud de las ventanas que se usó en las pruebas para la TFDG se eligió en base a analizar las señales con distintos valores de SD y elegir el valor que mostrará mejor los espectros.

Las señales de vibración fueron adquiridas durante el transitorio de arranque de los motores bajo las cinco condiciones de operación distintas, es decir, el motor sano, una barra del 

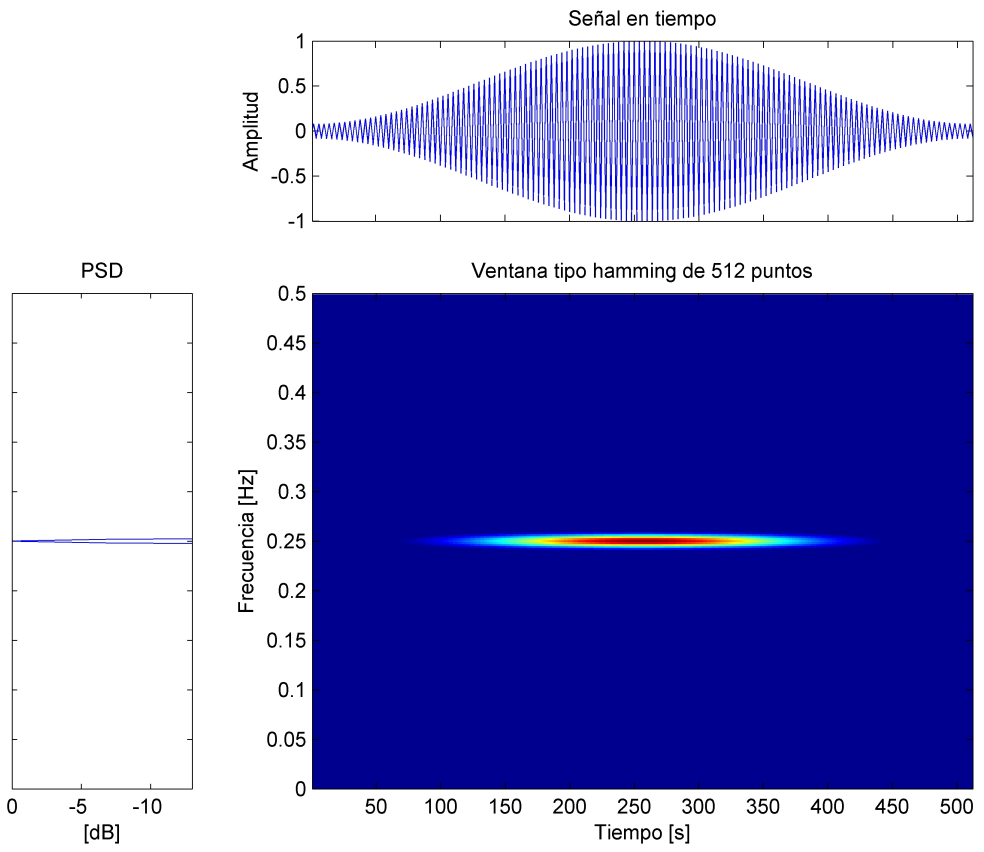

Figura 5.17: Ventana Hamming de 512 puntos.

rotor rota, dos barras del rotor rotas, desbalance y defectos en el rodamiento. Los mejores resultados se obtienen a partir de la señal de vibración en el eje $z$, Az, y esto se debe, como se ha comentado anteriormente, a que las vibraciones en un motor de inducción son típicamente vibraciones radiales. Los resultados se presentan en cuatro casos de estudio y se realiza un análisis cualitativo sobre la condición de operación del motor.

\subsubsection{Caso de estudio: motor sano}

La Figura 5.20 presenta la descomposición tiempo-frecuencia STFT, TFDG, TFMS y MUSIC de la señal de vibración en el eje z para el motor sano. Para este caso de estudio, la banda de frecuencias entre $400 \mathrm{~Hz}$ y $700 \mathrm{~Hz}$ tiene algunas variaciones apenas perceptibles asociadas con excentricidades preexistentes en el motor, las cuales sólo son perceptibles después del transitorio de arranque cuando se alcanza el régimen de estado estable. No hay otros 

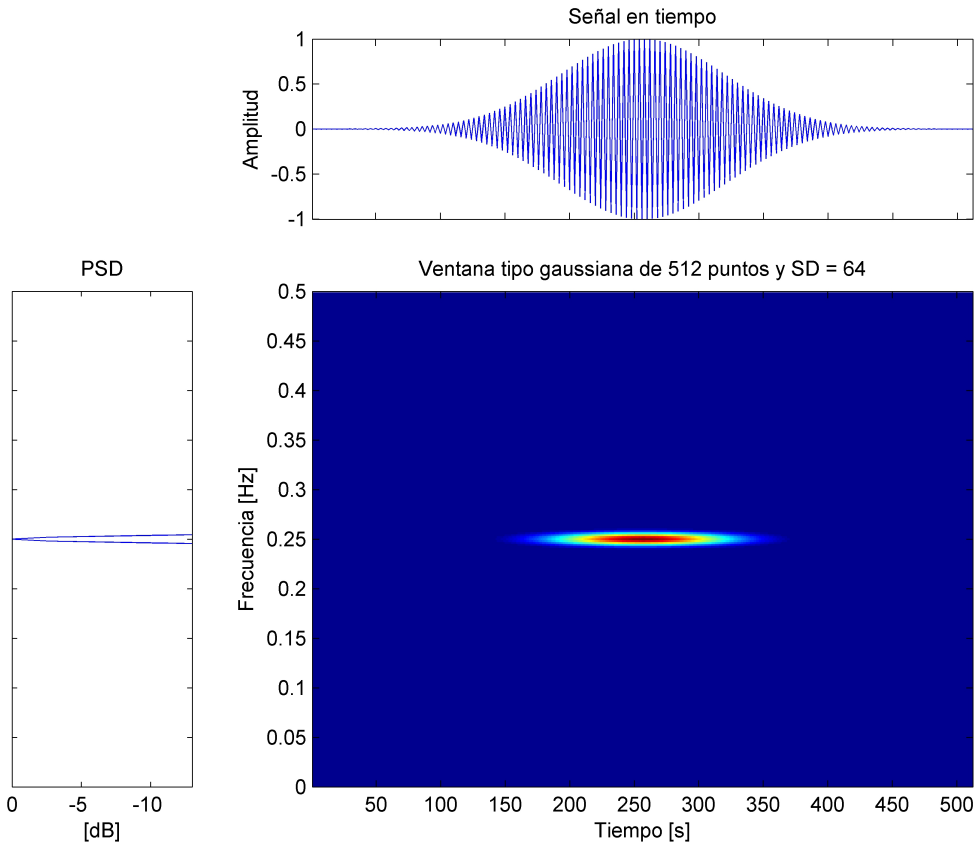

Figura 5.18: Ventana gaussiana de 512 puntos.

componentes de frecuencia significativa en los espectros para el caso de estudio del motor sano, y estas representaciones tiempo-frecuencia de las vibraciones del motor sano serán la referencia para determinar las condiciones de falla en el motor.

\subsubsection{Caso de estudio: Barras rotas}

Como ya se ha mencionado, la falla de barras rotas se puede detectar observando la evolución de la frecuencia asociada a esta falla en la gráfica tiempo-frecuencia. A partir de la ecuación (2.7), y los espectros obtenidos; se observa que para $k=11$, y $n=1$ existe un armónico $(617.1 \mathrm{~Hz})$ asociado a la falla de barras rotas cercano a la frecuencia de 640 $\mathrm{Hz}$ que se observa en los espectros del caso del motor sano. La Figura 5.21 (a) muestra el espectro obtenido con la STFT, además hay un ancho de banda de frecuencias de amplitud significativa alrededor de 620-655 Hz; pero el armónico $(617.1 \mathrm{~Hz}$ ) de la falla de barras rotas 

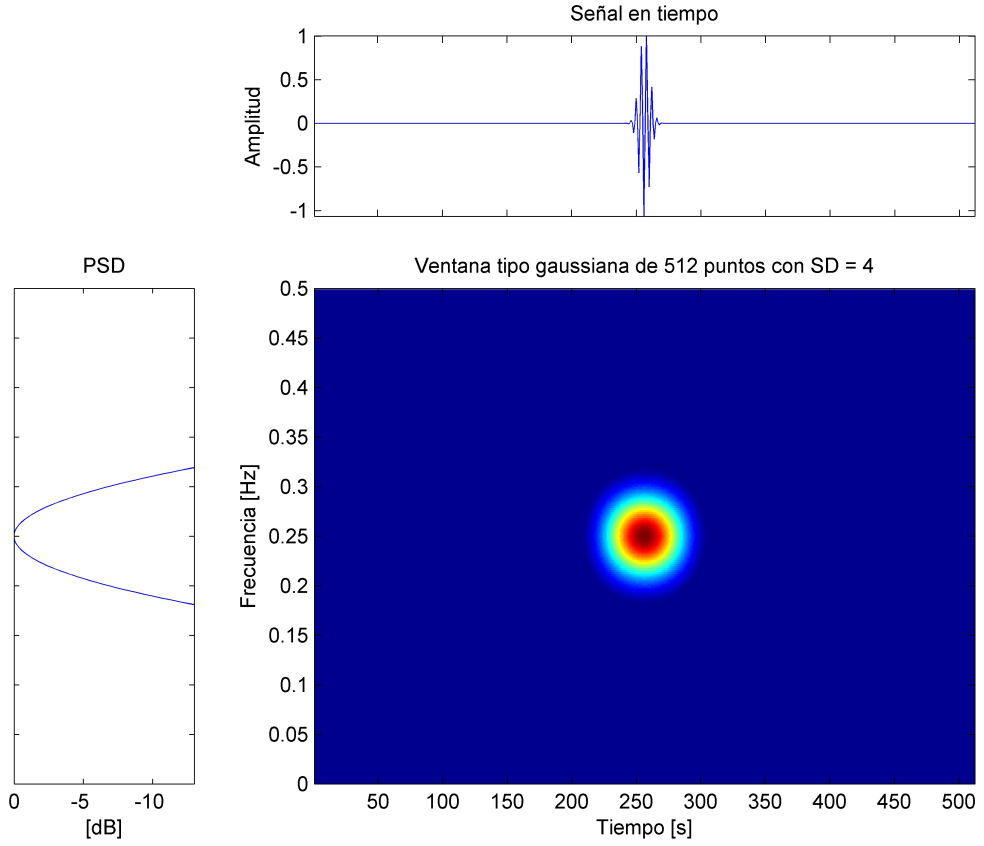

Figura 5.19: Ventana gaussiana de 512 puntos.

no se detecta con claridad. La Figura 5.21 (b) presenta el espectro obtenido con la TFDG, y la frecuencia más significativa es de alrededor de $640 \mathrm{~Hz}$; por lo que no se detecta el armónico. La Figura 5.21 (c) muestra el espectro obtenido con la TFMS, este es el peor método porque el ancho de banda de frecuencia con gran amplitud está situado alrededor de 610-690 $\mathrm{Hz}$; por lo que no se detecta la frecuencia asociada a las barras rotas. La Figura 5.21 (d) muestra el espectro calculado con MUSIC en el que el armónico con valor de $617.1 \mathrm{~Hz}$ asociado a la falla de barras rotas se detecta claramente hacia el final del transitorio de arranque y el comienzo del estado estacionario (marcado con una flecha punteada en blanco).

La Figura 5.22 muestra los espectros de la descomposición tiempo-frecuencia de la STFT, TFDG, TFMS y MUSIC para el caso de dos barras del rotor rotas. Para este caso de estudio, empleando la ecuación (2.7), la frecuencia de falla cuando $k=2$ y $n=4$ es igual a $87 \mathrm{~Hz}$ y el otro armónico $k=5$, y $n=3$ es igual a $263.7 \mathrm{~Hz}$, ambos armónicos están presentes 

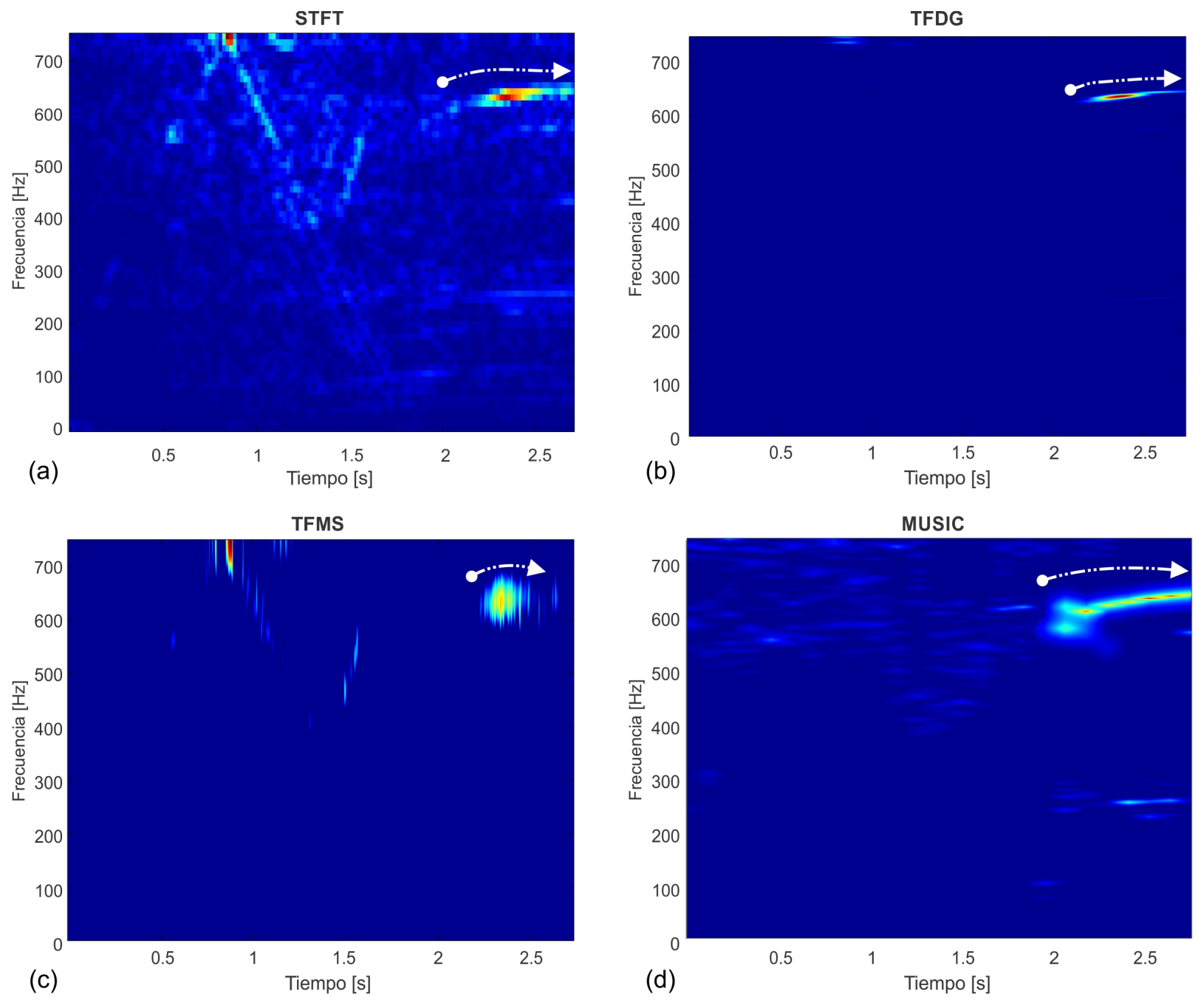

Figura 5.20: Descomposiciones tiempo-frecuencia para un motor sano. A) STFT. B) TFDG. C) TFMS. D) MUSIC.

en la mayoría de los espectros obtenidos (marcados con una flecha punteada en blanco). Sin embargo, estos armónicos no se observan claramente en las representaciones obtenidas con la STFT (Figura 5.22 (a)) y la TFMS (Figura 5.22 (c)) como en los otros dos métodos. La Figura 5.22 (b) muestra el espectro obtenido con la TFDG; en la representación obtenida con este método, ambas frecuencias se observan mejor con menos interferencia de ruido, y estas 

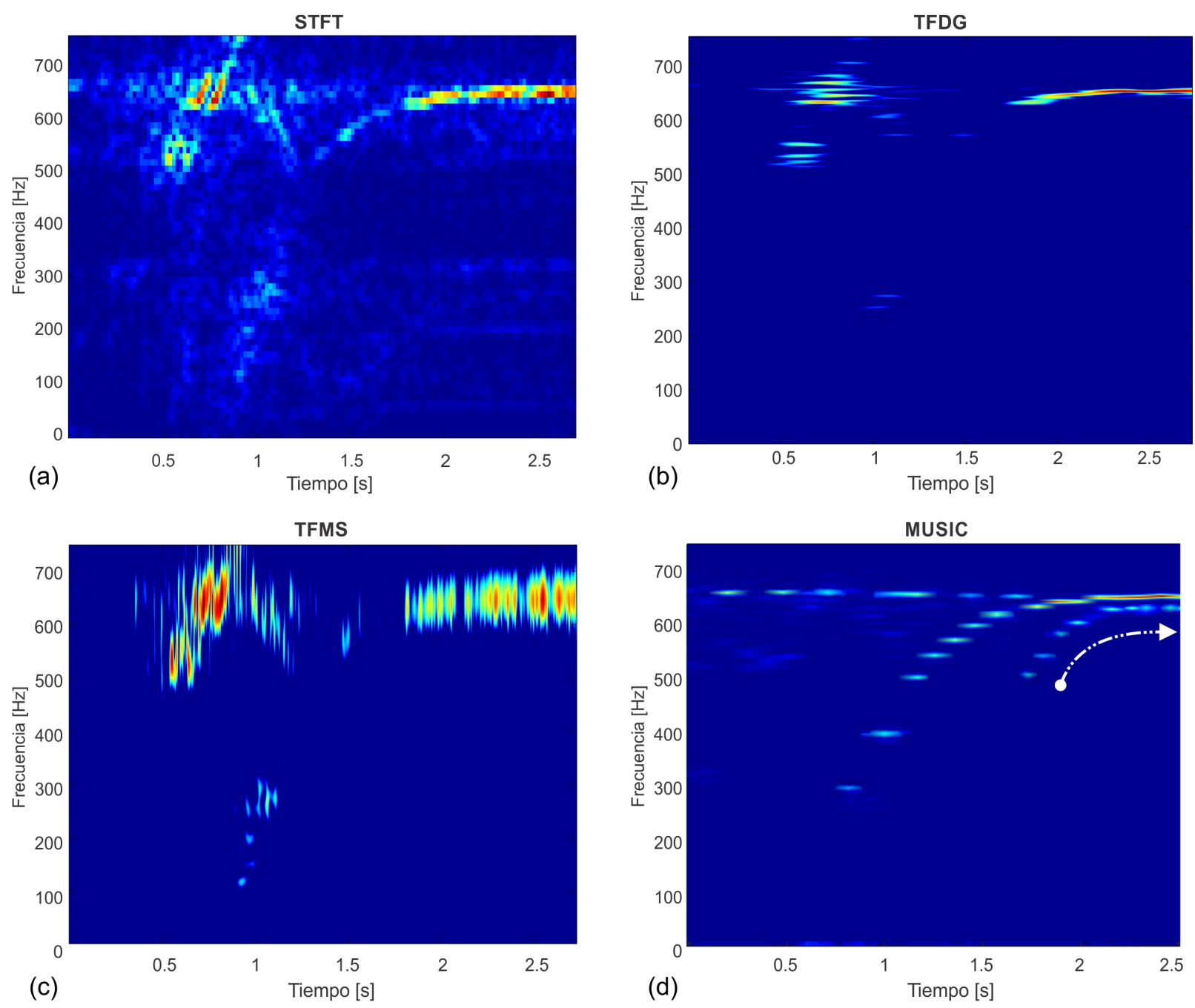

Figura 5.21: Descomposiciones tiempo-frecuencia para una barra del rotor rota. A) STFT. B) TFDG. C) TFMS. D) MUSIC.

frecuencias muestran una mejor resolución que en la STFT. La Figura 5.22 (c) muestra el espectro obtenido con la TFMS; donde la frecuencia de falla de barras rotas sólo se observa ligeramente durante el inicio del estado estacionario. La Figura 5.22 (d) presenta el espectro obtenido con MUSIC, este método es el que proporciona la mejor visualización para el caso estudiado, la frecuencia de falla y sus armónicos $(87 \mathrm{~Hz}$ y $263.7 \mathrm{~Hz}$ ) son claramente observados 
durante el transitorio de arranque y en estado estable. Pero además el armónico situado en 617.1 $\mathrm{Hz}$ se detecta ligeramente durante el comienzo del régimen de estado estacionario.
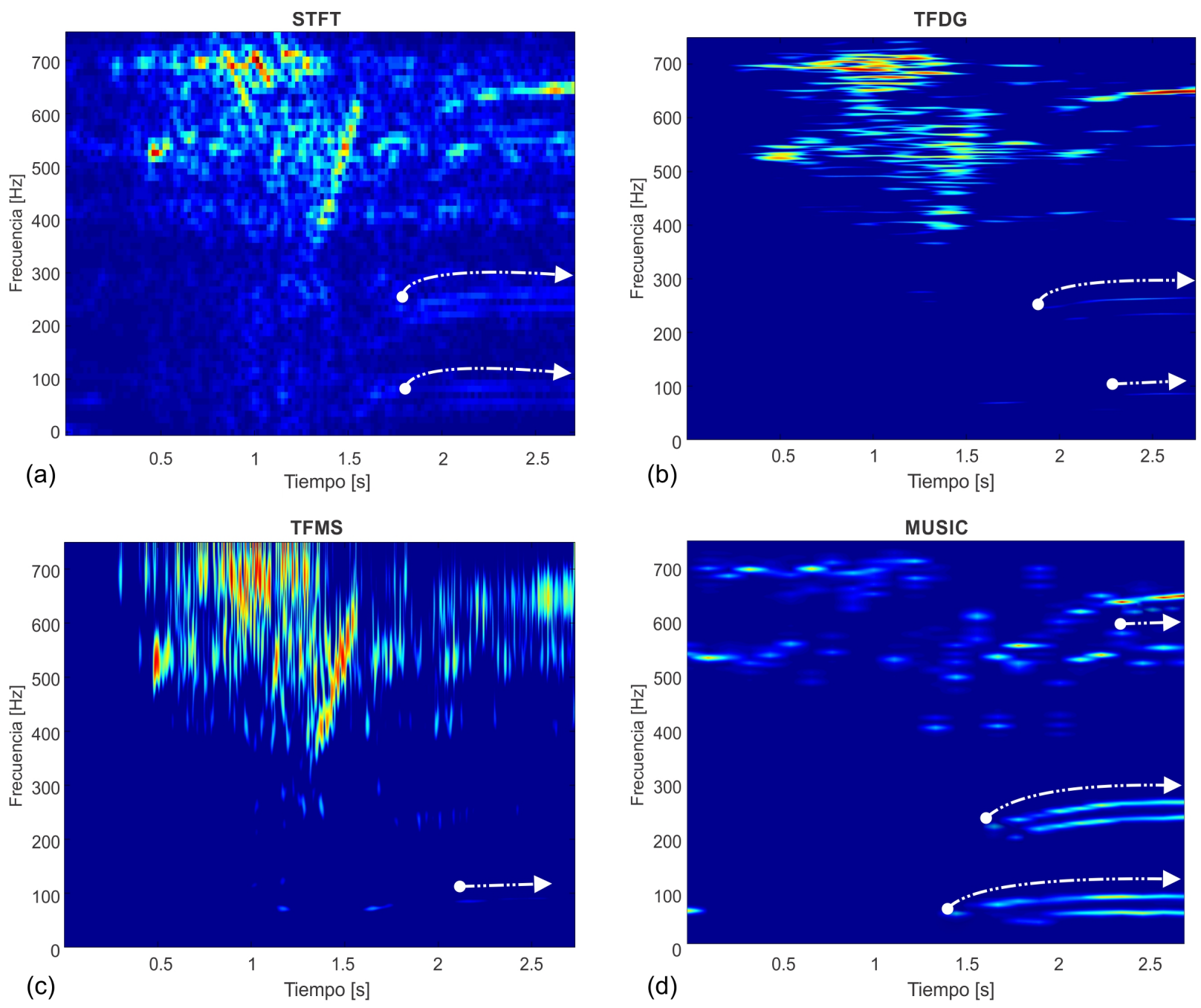

Figura 5.22: Espectros obtenidos con las descomposiciones tiempo-frecuencia para dos barras del rotor rotas. A) STFT. B) TFDG. C) TFMS. D) MUSIC. 


\subsubsection{Caso de estudio: Desbalance}

La Figura 5.23 muestra el análisis realizado con la STFT, TFDG, TFMS y MUSIC para el caso del motor con desbalance. En este caso, la velocidad del motor en revoluciones por minuto corresponde a un componente de frecuencia situado en $f_{r}=56.7 \mathrm{~Hz}$.
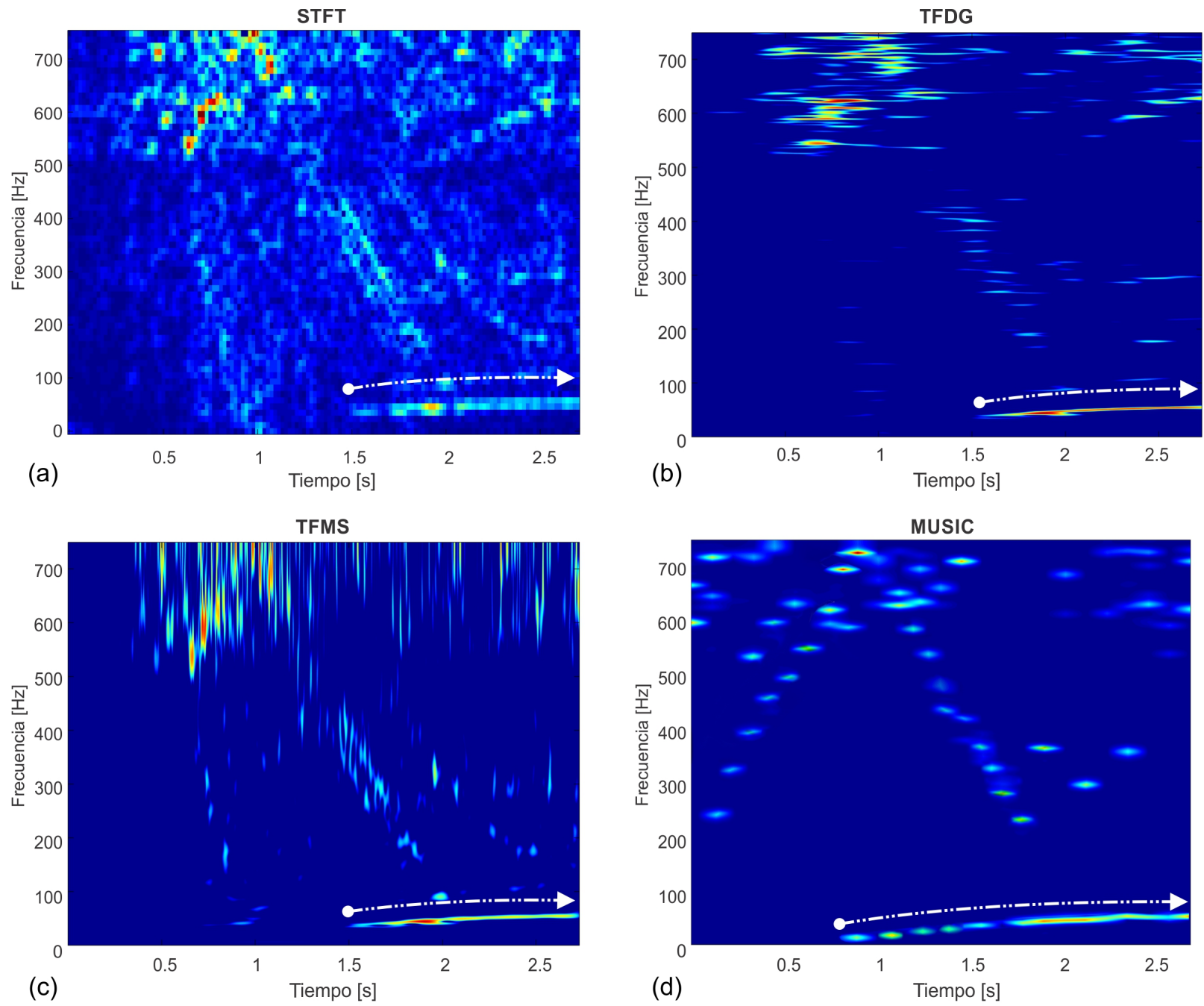

Figura 5.23: Espectros obtenidos con las descomposiciones tiempo-frecuencia para el caso de desbalance. A) STFT. B) TFDG. C) TFMS. D) MUSIC. 
Como se ve en la Figura 5.23, hay un componente de frecuencia alrededor de $56.7 \mathrm{~Hz}$ que está claramente definido. Este componente de frecuencia está asociado con la velocidad del rotor y su valor incrementado apunta hacia la presencia de desbalance en la condición de funcionamiento. Se puede observar que en el caso sano no hay componentes de frecuencia significativa a $56.7 \mathrm{~Hz}$ en los espectros tiempo-frecuencia, lo que demuestra la presencia de esta falla. Para todos los métodos de análisis: STFT, TFDG, TFMS y MUSIC, la frecuencia asociada con este fallo es claramente visible (marcada con una flecha punteada color blanco). Esta falla también contribuye a la formación de picos en la banda de frecuencias asociada con la excentricidad del motor, pero lo que hace posible la identificación es un aumento en la amplitud del componente espectral que está asociado con la rotación del rotor, y MUSIC proporciona la mejor visualización de la componente de frecuencia relacionada con la falla.

\subsubsection{Caso de estudio: Defectos en el rodamiento}

La Figura 5.24 representa los espectros de descomposición tiempo-frecuencia STFT, TFDG, TFMS y MUSIC para el caso de defectos en el rodamiento. Como se ha mencionado anteriormente, un defecto en la pista exterior provoca un impulso cada vez que un elemento rodante contacta con el defecto. Por lo tanto, la frecuencia relacionada con esta falla está asociada a la rotación del eje, y la frecuencia de falla, $f_{B P O F}$, se sitúa en $174.1 \mathrm{~Hz}$. MUSIC es el único método capaz de detectar esta frecuencia en el tercer armónico $(522 \mathrm{~Hz})$ al final del transitorio de arranque y el comienzo del régimen de estado estacionario.

La Tabla 5.6 muestra la comparación de detectabilidad en dB para todas las metodologías estudiadas. La detectabilidad se calcula como la relación de amplitud (en dB) entre la frecuencia asociada a la falla sobre la frecuencia en el espectro correspondiente al motor sano. El estudio comparativo se resume en la Tabla 5.6. El estudio de estas técnicas proporciona una evaluación de su efectividad para la detección de fallas en motores de inducción. La Tabla 5.6 muestra que MUSIC proporciona una mejor detectabilidad para las tres fallas estudiadas en un motor de inducción durante su transitorio de arranque. 

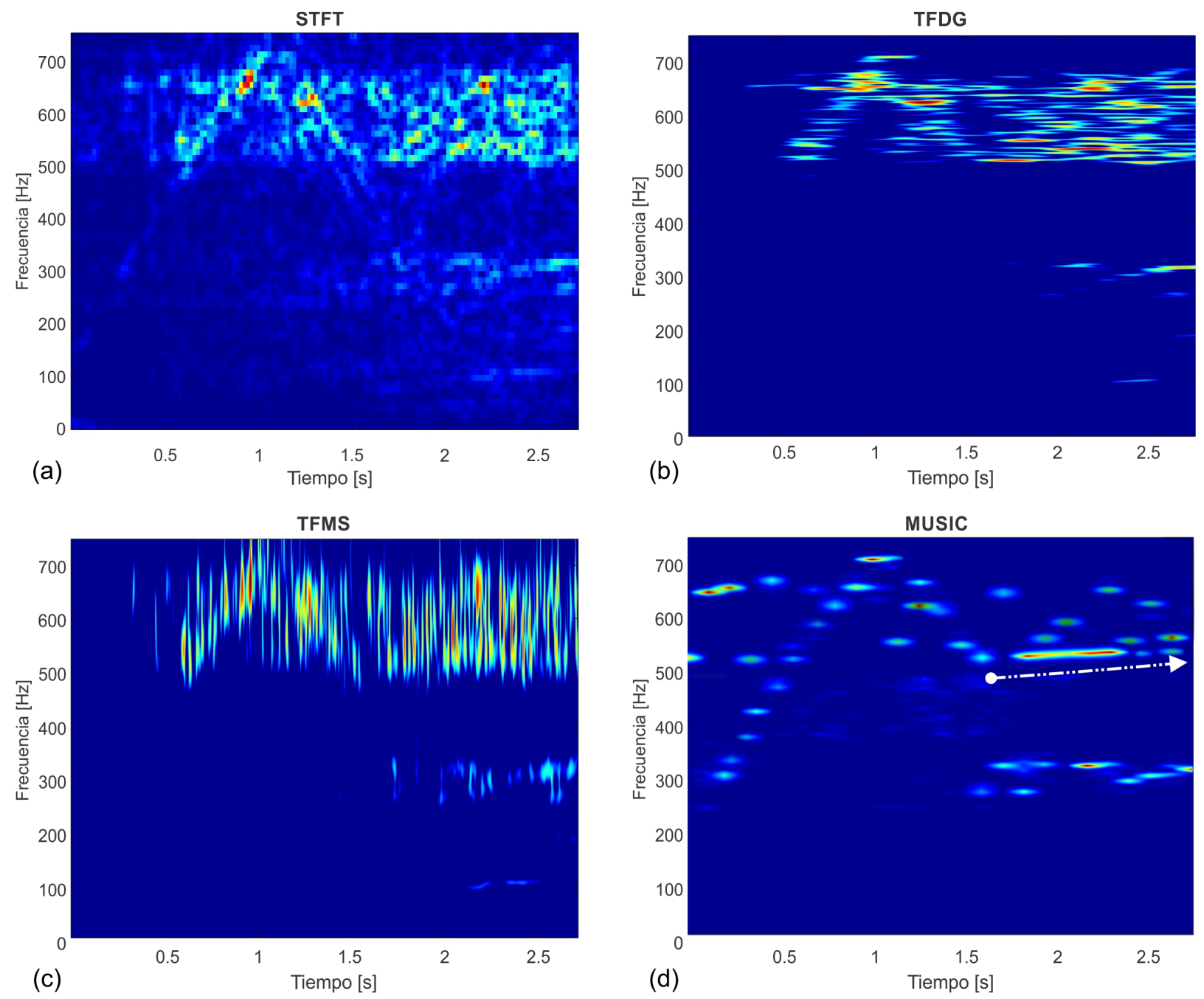

Figura 5.24: Espectros obtenidos de la descomposición tiempo-frecuencia para el caso de defectos en el rodamiento. A) STFT. B) TFDG. C) TFMS. D) MUSIC. 
Tabla 5.6: Detectabilidad en dB para el estudio comparativo de técnicas para la identificación de fallas en un motor de inducción.

\begin{tabular}{|l|c|c|c|c|}
\hline Condición del motor & STFT & TFDG & TFMS & MUSIC \\
\hline \hline Sano & 10 & 7 & 11 & 12 \\
\hline BRB & 12 & 9 & 13 & 21 \\
\hline UNB & 15 & 10 & 6 & 28 \\
\hline BDF & 16 & 12 & 21 & 32 \\
\hline
\end{tabular}




\section{Capítulo 6}

\section{Conclusiones y prospectivas}

En esta tesis se presentó una nueva metodología para el diagnóstico de fallas en motores de inducción. Esta metodología se aplica a señales capturadas durante el funcionamiento del motor en estado estable. Además, se presentó un estudio de técnicas de análisis espectral tiempo-frecuencia aplicadas al diagnóstico de motores de inducción durante el transitorio de arranque. En las siguientes dos secciones se detallan las conclusiones y aportaciones de las metodologías desarrolladas.

\subsection{Conclusiones del análisis en el estado estable}

En la presente tesis se proporcionó una nueva metodología de diagnóstico de motores de inducción analizando señales de sonido capturadas durante el funcionamiento en estado estable del motor. Los resultados obtenidos con el análisis de las señales de sonido se corroboraron con el análisis de señales de vibración. Esta corroboración de resultados se justifica debido a la gran aceptación que tiene el análisis de vibraciones y que constituye una de las metodologías más ampliamente utilizada en campo para el diagnóstico de fallas en motores. Las aportaciones hechas con el análisis en estado estable y con la metodología propuesta en este trabajo son las siguientes:

- Se demostró la viabilidad de detectar fallas, tales como una y dos barras del rotor rotas, defectos en la pista externa de rodamientos y desbalance 


\section{mecánico, en motores de inducción que operan en estado estacionario ana- lizando señales de sonido.}

Los resultados que se obtuvieron analizando señales de vibración corroboran los resultados que se obtuvieron analizando señales de sonido. En este trabajo se demostró que analizar señales de sonido es una técnica para el diagnóstico de motores tan efectiva como lo es el análisis de señales de vibración. Este hecho representa una aportación significativa debido a que esta metodología resulta extremadamente útil en los casos en que las señales de sonido son las únicas señales disponibles. Asimismo, el análisis de las señales sonoras es una técnica no invasiva y es muy fácil de hacer una implementación para la captura de las señales de sonido, lo que resulta en una gran ventaja ya que no es necesario intervenir de ninguna manera con el funcionamiento del motor.

- Otra de las aportaciones es el uso de la CEEMD en combinación con el calculo del marginal en frecuencia de la TFDG para separar la señal en IMFs.

Gracias a la CEEMD es posible seleccionar aquellas IMFs específicas que contienen la información necesaria para detectar las fallas y eliminar, al mismo tiempo, componentes de la señal no deseados. La CEEMD se utiliza como etapa de preprocesamiento y mejora la estimación del espectro obtenido al hacer el cálculo del marginal en frecuencia.

- La aplicación del cálculo del marginal en frecuencia de la representación de Gabor para obtener el espectro de las señales.

La elección del cálculo del marginal en frecuencia de la representación de Gabor es novedoso y se justificó por los siguientes hechos: (i) tiene una mejor tolerancia al ruido que la FFT; (ii) el espectro resultante es suavizado, lo que facilita su interpretación; (iii) no produce frecuencias espurias, como si lo hace MUSIC, la cual es una desventaja presente en ese método. Como resultado, las características de los espectros obtenidos facilitan la observación e identificación de componentes espectrales asociados con las fallas analizadas.

UG - UVa

Paulo Antonio Delgado Arredondo 
Sin embargo, y a pesar de las ventajas, la metodología propuesta tiene algunas limitaciones. La primera está asociada con el uso de la CEEMD. En esta descomposición no es posible conocer a priori el número de modos en los que se descompone la señal y qué modo o IMF contendrá también las componentes espectrales de interés. Todo esto hace necesario analizar cada IMF, o la mayoría de ellas, para identificar el contenido espectral. Hay otra limitación que también está presente en muchos trabajos relacionados con la detección de fallas en motores de inducción. La localización de las frecuencias asociadas a la falla depende del conocimiento de la velocidad del motor o del deslizamiento. En aplicaciones de campo, la velocidad del motor no siempre está disponible y debe estimarse mediante la identificación de los armónicos de ranura o de los componentes de frecuencia asociados a la excentricidad mixta.

\subsection{Conclusiones del análisis en el transitorio de arran- que}

En esta tesis también se presentó un análisis y validación de técnicas para su utilización en la detección de fallos en motores de inducción con distintos tipos de fallas y sano durante su funcionamiento en el transitorio de arranque. Este análisis se hizo en señales de vibración. La evaluación de las técnicas se hizo a partir de la localización de componentes espectrales asociados a las fallas después de la descomposición tiempo-frecuencia de la señal de vibraciones. En este sentido, las investigaciones realizadas anteriormente no son numerosas y la aportación del estudio de las metodologías aplicables a la detección de fallas en motores en el transitorio de arranque es significativa. Las aportaciones hechas con el estudio de estas metodologías se detallan a continuación:

- Se demostró la viabilidad de detectar fallas en motores de inducción durante el transitorio de arranque utilizando las técnicas de análisis espectral tiempo-frecuencia propuestas en este trabajo, aplicadas a señales de vibración.

UG - UVa

Paulo Antonio Delgado Arredondo 
Este punto es importante debido a que amplía el horizonte de técnicas que pueden implementarse durante el transitorio de un motor de inducción y además de que este estudio aumenta la posibilidad de aplicaciones ya que puede ser utilizable para analizar cualquier fenómeno que sea transitorio.

- Las técnicas propuestas en este trabajo son aplicables cuando no se cuenta con señales de corriente y sólo se tienen señales de vibración.

Este conjunto de técnicas de análisis es importante debido a que representa una opción más en la detección de fallos en motores de inducción, esto debido a que en ocasiones la única información con la que se cuenta son las vibraciones de un motor.

- Las técnicas propuestas en este trabajo son aplicables durante el transitorio de arranque de motores de inducción.

Además de las aportaciones anteriores, este análisis es en el transitorio de arranque del motor lo cual aumenta las posibilidades de diagnosticar un motor cuando se dé el caso de contar sólo con este tipo de información, ya que la gran mayoría de metodologías de diagnóstico se enfocan al diagnóstico de motores durante su funcionamiento en el estado estable.

- Las técnicas estudiadas para el análisis de señales transitorias de motores de inducción son aplicables a cualquier fenómeno transitorio.

A la par de su aplicación para el diagnóstico de motores, las técnicas que se estudian son aplicables al análisis de señales transitorias sin importar cuál sea su origen, hecho que aumenta sustancialmente el campo de aplicación y utilización del trabajo realizado en esta tesis.

- Este conjunto de técnicas de descomposición tiempo-frecuencia también tiene aplicaciones en motores que no se alimentan directamente de la red eléctrica.

En tales casos, cuando los motores se alimentan con inversores, no se recomienda el análisis de las señales de corriente debido a la mala calidad de la señal de corriente que se obtiene 
como consecuencia del uso de controladores de velocidad lo cual hace que disminuya sustancialmente la relación señal/ruido. Del mismo modo, con el uso de estas técnicas aplicadas a señales de vibración se pueden detectar fallas como se lograría con la técnica MCSA aplicada para el diagnóstico de motores de inducción. La técnica de descomposición tiempo-frecuencia que proporciona los mejores resultados es MUSIC, que tiene mayor resolución en frecuencia y es capaz de detectar las frecuencias y armónicos asociados a las fallas en señales de vibración con alto contenido de ruido. Sin embargo tiene el inconveniente de la aparición de frecuencias espurias.

\subsection{Prospectivas}

Con el trabajo realizado en esta tesis se abre una amplia gama de posibilidades para trabajos futuros. Entre las posibles líneas de investigación y prospectivas de aplicaciones que se derivan del trabajo realizado se encuentran las siguientes:

- La metodología propuesta para el análisis en estado estable y las técnicas de descomposición tiempo-frecuencia que se analizan en este trabajo pueden ser aplicadas para el diagnóstico de otro tipo de fallas presentes en motores de inducción como por ejemplo la presencia de contaminación en los rodamientos.

- La metodología propuesta para el análisis en estado estable y las técnicas de descomposición tiempo-frecuencia que se analizan en este trabajo también pueden ser aplicadas para el diagnóstico de fallas en motores de inducción alimentados con inversor.

- La metodología que se propuso para el análisis de señales de sonido puede ser aplicada para identificar fallas en otro tipo de máquinas o sistemas en los cuales el sonido se emita como consecuencia de las condiciones de operación.

- Mejorar la aportación de este trabajo justificando desde un punto de vista teórico las frecuencias de las componentes de avería en un espectro de sonido. Esto permitiría asentar que no son específicas de este caso y que el método es general. 
- Las técnicas para la descomposición tiempo-frecuencia que se estudiaron en este trabajo pueden ser aplicadas a cualquier fenómeno transitorio sin importar su origen.

- Para un mayor desarrollo de la metodología y técnicas que en este trabajo se proponen, también se puede incluir un sistema de clasificación para la detección automática de fallas.

- Además, el análisis aplicado a la fusión de datos de los sensores de aceleración, sonido y corriente podría producir una metodología mejorada para la detección de fallas en motores de inducción. 


\section{Contribuciones}

Esta tesis es el resultado del trabajo realizado en los programas de doctorado en Ingeniería Eléctrica en la Universidad de Guanajuato y en el programa de doctorado en Ingeniería Industrial en la Universidad de Valladolid. El desarrollo de los programas se hizo bajo un acuerdo de cotutela en la modalidad de doble titulación celebrado entre ambas universidades.

De las actividades realizadas durante el desarrollo de los programas de doctorado, se verificó la validez de algunas técnicas de análisis espectral para la localización de fallas en señales de vibración obtenidas de un motor, de este trabajo se obtuvo como resultado la publicación de un articulo. Este artículo fue publicado en el journal Shock and Vibration el cual está indexado al JCR (acrónimo de Journal Citation Report) y tiene un factor de impacto de 0.88 (Q3), la información completa del artículo es la siguiente:

\section{P.A. Delgado-Arredondo, A. Garcia-Perez, D. Moriñigo-Sotelo, R.A. Osornio- Rios, J.G. Avina-Cervantes, H. Rostro-Gonzalez and R.J. Romero-Troncoso. Comparative study of time-frequency decomposition techniques for fault detec- tion in induction motors using vibration analysis during startup transient. Shock and Vibration, 2015(2015):1-14, 2015.}

Además, se destaca la realización de una estancia de investigación en la Universidad de Valladolid de duración de un año, durante el periodo comprendido entre el mes de septiembre del año 2015 al mes de septiembre del año 2016. Durante este periodo se concluyó con el diseño de un banco de pruebas y con la experimentación para la captura de señales y se 
verificó la validez de una metodología para la localización de fallas analizando señales de sonido y vibración obtenidas de un motor, de este trabajo se obtuvo como resultado la publicación de otro artículo. Este artículo fue publicado en el journal Mechanical Systems and Signal Processing el cual esta indexado al JCR y tiene un factor de impacto de 2.771 (Q1). La información completa del artículo es la siguiente:

P.A. Delgado-Arredondo, D. Moriñigo-Sotelo, R.A. Osornio-Rios, J.G. AvinaCervantes, H. Rostro-Gonzalez and R.J. Romero-Troncoso. Methodology for fault detection in induction motors via sound and vibration signals. Mechanical Systems and Signal Processing 83(2017):568-589, 2017. 


\section{Referencias}

[1] H. Falkner and H. Shane. Walking the Torque: Proposed Work Plan for EnergyEfficiency Policy Opportunities for Electric Motor-Driven Systems. IEA Energy Papers. OECD Publishing, Paris, 2011.

[2] A.V. Oppenheim, A.S. Willsky, S.H. Nawab, and G.M. Hernández. Señales y sistemas. Pearson Educación. Pearson Educación, 1998.

[3] S. Nandi, H. A. Toliyat, and X. Li. Condition monitoring and fault diagnosis of electrical motors - A review. IEEE Transactions on Energy Conversion, 20(4):719-729, 2005.

[4] W. Deleroi. Broken bar in a squirrel-cage rotor of an induction motor - Description by superimposed fault-currents. Archiv für electrotechnik, 67:91-99, 1984.

[5] W.T. Thomson and I.D. Stewart. On-line current monitoring for fault diagnosis in inverter fed induction motors. IEE Conference Publication, 1988(291):432-435, 1988.

[6] I. Georgakopoulos, E. Mitronikas, and A. Safacas. Broken bar detection using inverter currents in induction motor drives. 2010 International Symposium on Power Electronics, Electrical Drives, Automation and Motion, SPEEDAM 2010, 2010(4):1030-1035, 2010 .

[7] S.K. Kim and J.K. Seok. High-frequency signal injection-based rotor bar fault detection of inverter-fed induction motors with closed rotor slots. IEEE Transactions on Industry Applications, 47(4):1624-1631, 2011. 
[8] T.W. Chua, W.W. Tan, Z.X. Wang, and C.S. Chang. Hybrid time-frequency domain analysis for inverter-fed induction motor fault detection. IEEE International Symposium on Industrial Electronics, pages 1633-1638, 2010.

[9] J. Pons-Llinares, J. Antonino-Daviu, J. Roger-Folch, D. Morinigo-Sotelo, and O. Duque-Perez. Eccentricity diagnosis in Inverter - Fed Induction Motors via the Analytic Wavelet Transform of transient currents. In The XIX International Conference on Electrical Machines - ICEM 2010, pages 1-6. IEEE, sep 2010.

[10] O. Duque-Perez, D. Morinigo-Sotelo, M. Perez-Alonso, A. Rodriguez-Carrascal, and L.A. Garcia-Escudero. Identification of eccentricity related-faults characteristic frequencies in induction motors using a programmable power source. 8th IEEE Symposium on Diagnostics for Electrical Machines, Power Electronics and Drives, SDEMPED 2011 A4 - IEEE Power Electronic Society (PELS), pages 468-473, 2011.

[11] I.P. Georgakopoulos, E.D. Mitronikas, and A.N. Safacas. Detection of Induction Motor Faults in Inverter Drives Using Inverter Input Current Analysis. IEEE Transactions on Industrial Electronics, 58(9):4365-4373, sep 2011.

[12] D. Ece and M. Basaran. Condition monitoring of speed controlled induction motors using wavelet packets and discriminant analysis. Expert Systems with Applications, 38(7):8079-8086, 2011.

[13] K. Yahia, J.M. Cardoso, S.E. Zouzou, and S. Gueddidi. Broken rotor bars diagnosis in an induction motor fed from a frequency converter: Experimental research. International Journal of Systems Assurance Engineering and Management, 3(1):40-46, 2012.

[14] M. Fernandez-Temprano, P.E. Gardel-Sotomayor, O. Duque-Perez, and D. MorinigoSotelo. Broken bar condition monitoring of an induction motor under different supplies using a linear discriminant analysis. In 2013 9th IEEE International Symposium on Diagnostics for Electric Machines, Power Electronics and Drives (SDEMPED), pages 162-168. IEEE, aug 2013. 
[15] S. Mitra and C. Koley. Vibration signal analysis of induction motors used in process control operation. 2013 IEEE 1st International Conference on Condition Assessment Techniques in Electrical Systems (CATCON), pages 286-291, 2013.

[16] Z. Wang, C.S. Chang, and Y. Zhang. A feature based frequency domain analysis algorithm for fault detection of induction motors. In 2011 6th IEEE Conference on Industrial Electronics and Applications, pages 27-32. IEEE, jun 2011.

[17] R.J. Romero-Troncoso, D. Morinigo-Sotelo, O. Duque-Perez, P.E. Gardel-Sotomayor, R.A. Osornio-Rios, and A. Garcia-Perez. Early broken rotor bar detection techniques in VSD-fed induction motors at steady-state. 2013 9th IEEE International Symposium on Diagnostics for Electric Machines, Power Electronics and Drives, SDEMPED 2013 A4 - 'Generalitat Valenciana-Conselleria d'Educacio, Cultura i Esport'; IEEE Power Electronics Society (PELS); SIEMENS; Universitat Pol, pages 105-113, 2013.

[18] J. Pons-Llinares, J. Antonino-Daviu, J. Roger-Folch, D. Morinigo-Sotelo, and O. Duque-Pérez. Mixed eccentricity diagnosis in Inverter-Fed Induction Motors via the Adaptive Slope Transform of transient stator currents. Mechanical Systems and Signal Processing, 48(1-2):423-435, 2014.

[19] R.J. Romero-Troncoso, A. Garcia-Perez, D. Morinigo-Sotelo, O. Duque-Perez, R.A. Osornio-Rios, and M.A. Ibarra-Manzano. Rotor unbalance and broken rotor bar detection in inverter-fed induction motors at start-up and steady-state regimes by highresolution spectral analysis. Electric Power Systems Research, 133(May):142-148, 2016.

[20] T. Yang, H. Pen, Z. Wang, and C.S. Chang. Feature knowledge based fault detection of induction motors through the analysis of stator current data. IEEE Transactions on Instrumentation and Measurement, 65(3):549-558, 2016.

[21] M.E.H. Benbouzid and G.B. Kliman. What stator current processing-based technique to use for induction motor rotor faults diagnosis? IEEE Transactions on Energy Conversion, 18(2):238-244, 2003. 
[22] G.K. Singh and S. Ahmed Saleh Al Kazzaz. Induction machine drive condition monitoring and diagnostic researchâa survey. Electric Power Systems Research, 64(2):145-158, 2003.

[23] B. Ayhan, H.J. Trussell, M.Y. Chow, and M.H. Song. On the use of a lower sampling rate for broken rotor bar detection with DTFT and AR-based spectrum methods. IEEE Transactions on Industrial Electronics, 55(3):1421-1434, 2008.

[24] W.T. Thomson and M. Fenger. Current signature analysis to detect induction motor faults. IEEE Industry Applications Magazine, 7(4):26-34, 2001.

[25] N.Q. Hu, L.R. Xia, F.S. Gu, and G.J. Qin. A novel transform demodulation algorithm for motor incipient fault detection. IEEE Transactions on Instrumentation and Measurement, 60(2):480-487, 2011.

[26] A. Garcia-Perez, R.J. Romero-Troncoso, E. Cabal-Yepez, and R.A. Osornio-Rios. The Application of High-Resolution Spectral Analysis for Identifying Multiple Combined Faults in Induction Motors. IEEE Transactions on Industrial Electronics, 58(5):20022010, may 2011.

[27] J.J. Rangel-Magdaleno, R.J. Romero-Troncoso, R.A. Osornio-Rios, E. Cabal-Yepez, and L.M. Contreras-Medina. Novel methodology for online half-broken-bar detection on induction motors. IEEE Transactions on Instrumentation and Measurement, 58(5):1690-1698, 2009.

[28] M. Riera-Guasp, J. Antonino-Daviu, J. Rusek, and J. Roger-Folch. Diagnosis of rotor asymmetries in induction motors based on the transient extraction of fault components using filtering techniques. Electric Power Systems Research, 79(8):1181-1191, 2009.

[29] J. Pons-Llinares, J.A. Antonino-Daviu, M. Riera-Guasp, M. Pineda-Sanchez, and V. Climente-Alarcon. Induction Motor Diagnosis Based on a Transient Current Analytic Wavelet Transform via Frequency B-Splines. IEEE Transactions on Industrial Electronics, 58(5):1530-1544, may 2011. 
[30] V. Ghorbanian and J. Faiz. A survey on time and frequency characteristics of induction motors with broken rotor bars in line-start and inverter-fed modes. Mechanical Systems and Signal Processing, 54-55:427-456, 2015.

[31] R. Miceli, Y. Gritli, A. Di Tommaso, F. Filippetti, and C. Rossi. Vibration signature analysis for monitoring rotor broken bar in double squirrel cage induction motors based on wavelet analysis. COMPEL - The international journal for computation and mathematics in electrical and electronic engineering, 33(5):1625-1641, aug 2014.

[32] P.C.M.L Filho, J.N. Brito, V.A.D. Silva, and R. Pederiva. Detection of electrical faults in induction motors using vibration analysis. Journal of Quality in Maintenance Engineering, 19(4):364-380, oct 2013.

[33] A. Prudhom, J.A. Antonino-Daviu, H. Razik, and V. Climente-Alarcon. Timefrequency vibration analysis for the detection of motor damages caused by bearing currents. Mechanical Systems and Signal Processing, pages 1-16, 2015.

[34] V. Climente-Alarcon, J.A. Antonino-Daviu, F. Vedreno-Santos, and R. PuchePanadero. Vibration transient detection of broken rotor bars by PSH sidebands. IEEE Transactions on Industry Applications, 49(6):2576-2582, 2013.

[35] R.K. Pate and V.K. Giri. Induction motor bearing fault diagnosis using cascaded EMD and DWT techniques. International Journal of Applied Engineering Research, 10(11):28317-28330, 2015.

[36] A. Bellini, C. Concari, G. Franceschini, C. Tassoni, and A. Toscani. Vibrations, currents and stray flux signals to asses induction motors rotor conditions. IECON Proceedings (Industrial Electronics Conference), 2(3):4963-4968, 2006.

[37] A. Garcia-Perez, R.J. Romero-Troncoso, E. Cabal-Yepez, R.A. Osornio-Rios, and J.A. Lucio-Martinez. Application of high-resolution spectral analysis for identifying faults in induction motors by means of sound. Journal of Vibration and Control, 18(11):15851594 , oct 2011. 
[38] F. Salazar-Villanueva and O.G. Ibarra-Manzano. Spectral analysis for identifying faults in induction motors by means of sound. In CONIELECOMP 2013, 23rd International Conference on Electronics, Communications and Computing, pages 149-153. IEEE, mar 2013.

[39] H. Akcay and E. Germen. Identification of acoustic spectra for fault detection in induction motors. In 2013 Africon, pages 1-5. IEEE, sep 2013.

[40] A. Glowacz. Diagnostics of DC and Induction Motors Based on the Analysis of Acoustic Signals. Measurement Science Review, 14(5):257-262, jan 2014.

[41] E. Germen, M. Başaran, and M. Fidan. Sound based induction motor fault diagnosis using Kohonen self-organizing map. Mechanical Systems and Signal Processing, 46(1):45-58, 2014.

[42] A.M. Al-Ghamd and D. Mba. A comparative experimental study on the use of acoustic emission and vibration analysis for bearing defect identification and estimation of defect size. Mechanical Systems and Signal Processing, 20(7):1537-1571, oct 2006.

[43] A. Choudhury and N. Tandon. Application of acoustic emission technique for the detection of defects in rolling element bearings. Tribology International, 33(1):39-45, jan 2000 .

[44] Y. He, X. Zhang, and M.I. Friswell. Defect Diagnosis for Rolling Element Bearings Using Acoustic Emission. Journal of Vibration and Acoustics, 131(6):061012, 2009.

[45] S. Al-Dossary, R.I.R Hamzah, and D. Mba. Observations of changes in acoustic emission waveform for varying seeded defect sizes in a rolling element bearing. Applied Acoustics, 70(1):58-81, 2009.

[46] J. Halme and P. Andersson. Rolling contact fatigue and wear fundamentals for rolling bearing diagnostics â state of the art. Proceedings of the Institution of Mechanical Engineers, Part J: Journal of Engineering Tribology, 224(4):377-393, jan 2010. 
[47] M. Elforjani and D. Mba. Accelerated natural fault diagnosis in slow speed bearings with Acoustic Emission. Engineering Fracture Mechanics, 77(1):112-127, jan 2010.

[48] M. Elforjani and D. Mba. Detecting natural crack initiation and growth in slow speed shafts with the Acoustic Emission technology. Engineering Failure Analysis, 16(7):2121-2129, 2009.

[49] W. Caesarendra, B. Kosasih, A. Kiet, H. Zhu, C.A.S. Moodie, and Q. Zhu. Acoustic emission-based condition monitoring methods : Review and application for low speed slew bearing. Mechanical Systems and Signal Processing, 72-73:134-159, 2016.

[50] W. Zhou, T.G. Habetler, and R.G. Harley. Bearing Condition Monitoring Methods for Electric Machines: A General Review. 2007 IEEE International Symposium on Diagnostics for Electric Machines, Power Electronics and Drives, pages 3-6, 2007.

[51] A. Gandhi, T. Corrigan, and L. Parsa. Recent Advances in Modeling and Online Detection of Stator Interturn Faults in Electrical Motors. IEEE Transactions on Industrial Electronics, 58(5):1564-1575, 2011.

[52] H. Su, K.T. Chong, and R. Ravi Kumar. Vibration signal analysis for electrical fault detection of induction machine using neural networks. Neural Computing and Applications, 20(2):183-194, 2011.

[53] S. Nandi, T.C. Ilamparithi, S.B. Lee, and D. Hyun. Detection of eccentricity faults in induction machines based on nameplate parameters. IEEE Transactions on Industrial Electronics, 58(5):1673-1683, 2011.

[54] E. Cabal-Yepez, A.G. Garcia-Ramirez, R.J. Romero-Troncoso, A. Garcia-Perez, and R.A. Osornio-Rios. Reconfigurable Monitoring System for Time-Frequency Analysis on Industrial Equipment Through STFT and DWT. IEEE Transactions on Industrial Informatics, 9(2):760-771, 2013. 
[55] M. Riera-guasp, J.A. Antonino-Daviu, J. Roger-folch, and M.P. Molina-Palomares. The Use of the Wavelet Approximation Signal as a Tool for the Diagnosis of Rotor Bar Failures. IEEE Transactions on Industry Applications, 44(3):716-726, 2008.

[56] J. Cusido, L. Romeral, J.A. Ortega, J.A. Rosero, and A. Garcia-Espinosa. Fault Detection in Induction Machines Using Power Spectral Density in Wavelet Decomposition. IEEE Transactions on Industrial Electronics, 55(2):633-643, 2008.

[57] P.S. Panigrahy, P. Konar, and P. Chattopadhyay. Broken bar fault detection using fused DWT-FFT in FPGA platform. In 2014 International Conference on Power, Control and Embedded Systems (ICPCES), pages 1-6. IEEE, dec 2014.

[58] I.S. Lee. HCI International 2011 - Posters' Extended Abstracts, volume 173 of Communications in Computer and Information Science. Springer Berlin Heidelberg, Berlin, Heidelberg, 2011.

[59] S.K. Ahamed, S. Karmakar, M. Mitra, and S. Sengupta. Diagnosis of induction motor faults due to broken rotor bar and rotor mass unbalance through discrete wavelet transform of starting current at no-load. Journal of Electrical Systems, 6(3):442-456, 2010.

[60] C. Rodriguez-Donate, R. Romero-Troncoso, E. Cabal-Yepez, A. Garcia-Perez, and R. Osornio-Rios. Wavelet-Based General Methodology For Multiple Fault Detection on Induction Motors at The Startup Vibration Transient. Journal of Vibration and Control, 17(9):1299-1309, 2011.

[61] K. Yahia, J.M. Cardoso, A. Ghoggal, and S.E. Zouzou. Induction motors airgapeccentricity detection through the discrete wavelet transform of the apparent power signal under non-stationary operating conditions. ISA transactions, 53(2):603-11, 2014.

[62] V.K. Giri. Broken rotor bar fault detection in induction motors using Wavelet Transform. 2012 International Conference on Computing, Electronics and Electrical Technologies (ICCEET), pages 1-6, 2012. 
[63] A. Saghafinia, S. Kahourzade, A. Mahmoudi, W.P. Hew, and M. Nasir Uddin. On line trained fuzzy logic and adaptive continuous wavelet transform based high precision fault detection of IM with broken rotor bars. In 2012 IEEE Industry Applications Society Annual Meeting, pages 1-8. IEEE, oct 2012.

[64] A. Saghafinia, S. Kahourzade, A. Mahmoudi, W.P. Hew, and M.N. Uddin. Broken Rotor Bar Fault Detection of 3-Phase Induction Motor Using Online Adaptive Continuous Wavelet Transform and Fuzzy Logic. INTERNATIONAL REVIEW OF ELECTRICAL ENGINEERING-IREE, 7(3, A):4383-4394, 2012.

[65] S. Das, C. Koley, P. Purkait, and S. Chakravorti. Wavelet aided SVM classifier for stator inter-turn fault monitoring in induction motors. IEEE PES General Meeting, PES 2010, pages 10-15, 2010.

[66] A. Pilloni, A. Pisano, M. Riera-Guasp, R. Puche-Panadero, and M. Pineda-Sanchez. Fault Detection in Induction Motors. In AC Electric Motors Control, pages 275-309. John Wiley \& Sons Ltd, 2013.

[67] Z. Feng, M. Liang, and F. Chu. Recent advances in time â frequency analysis methods for machinery fault diagnosis : A review with application examples. Mechanical Systems and Signal Processing, 38(1):165-205, 2013.

[68] J.A. Antonino-Daviu, M. Riera-Guasp, M. Pineda-Sanchez, and R.B. Perez. A critical comparison between DWT and Hilbert-Huang-based methods for the diagnosis of rotor bar failures in induction machines. IEEE Transactions on Industry Applications, 45(5):1794-1803, 2009.

[69] Z.K. Peng, P.W. Tse, and F.L. Chu. A comparison study of improved Hilbert-Huang transform and wavelet transform: Application to fault diagnosis for rolling bearing. Mechanical Systems and Signal Processing, 19(5):974-988, 2005.

[70] V. Climente-Alarcon, J.A. Antonino-Daviu, M. Riera-Guasp, and M. Vlcek. Induction Motor Diagnosis by Advanced Notch FIR Filters and the WignerâVille Distribution. IEEE Trans. Ind. Electron., 61(8):4217-4227, 2013. 
[71] S.S. Refaat, H. Abu-Rub, M.S. Saad, E.M. Aboul-Zahab, and A. Iqbal. ANN-based for detection, diagnosis the bearing fault for three phase induction motors using current signal. 2013 IEEE International Conference on Industrial Technology (ICIT), pages 253-258, 2013.

[72] V. Climente-Alarcon, J.A. Antonino-Daviu, M. Riera-Guaspa, R. Puche-Panaderoa, and L. Escobar. Application of the Wigner-Ville distribution for the detection of rotor asymmetries and eccentricity through high-order harmonics. Electric Power Systems Research, 91:28-36, 2012.

[73] V. Climente-Alarcon, J. Antonino-Daviu, M. Riera-Guasp, J. Pons-Llinares, J. RogerFolch, P. Jover-Rodriguez, and A. Arkkio. Transient tracking of low and high-order eccentricity-related components in induction motors via TFD tools. Mechanical Systems and Signal Processing, 25(2):667-679, 2011.

[74] V. Climente-Alarcon, J.A. Antonino-Daviu, M. Riera-Guasp, R. Puche, L. EscobarMoreira, N. Wiener, P. Jover-Rodriguez, and a. Arkkio. Diagnosis of stator shortcircuits through Wigner-Ville transient-based analysis. 2009 35th Annual Conference of IEEE Industrial Electronics, (1):1097-1102, 2009.

[75] M. Blödt, D. Bonacci, J. Regnier, M. Chabert, and J. Faucher. On-line monitoring of mechanical faults in variable-speed induction motor drives using the Wigner distribution. IEEE Transactions on Industrial Electronics, 55(2):522-533, 2008.

[76] S. Rajagopalan, J.M. Aller, J.A. Restrepo, T.G. Habetler, and R.G. Harley. Detection of rotor faults in brushless DC motors operating under nonstationary conditions. IEEE Transactions on Industry Applications, 42(6):1464-1477, 2006.

[77] S. Rajagopalan, J.A. Restrepo, J.M. Aller, T.G. Habetler, and R.G. Harley. Nonstationary Motor Fault Detection Using Recent Quadratic Time â Frequency Representations. IEEE Transactions on Industry Applications, 44(3):735-744, 2008. 
[78] Y. Yang, Z.K. Peng, G. Meng, and W.M. Zhang. Spline-Kernelled Chirplet Transform for the Analysis of Signals With Time-Varying Frequency and Its Application. IEEE Transactions on Industrial Electronics, 59(3):1612-1621, 2012.

[79] A. Garcia-Perez, R.J. Romero-Troncoso, E. Cabal-Yepez, R.A. Osornio-Rios, J.J. Rangel-Magdaleno, and H. Miranda. Startup current analysis of incipient broken rotor bar in induction motors using high-resolution spectral analysis. SDEMPED 2011 8th IEEE Symposium on Diagnostics for Electrical Machines, Power Electronics and Drives, pages 657-663, 2011.

[80] A.G. Garcia-Ramirez, R.A. Osornio-Rios, D. Granados-Lieberman, A. Garcia-Perez, and R.J. Romero-Troncoso. Smart sensor for online detection of multiple-combined faults in VSD-fed induction motors. Sensors (Basel, Switzerland), 12(9):11989-2005, 2012 .

[81] S. Guedidi, S. E. Zouzou, W. Laala, M. Sahraoui, and K. Yahia. Broken bar fault diagnosis of induction motors using MCSA and neural network. SDEMPED 2011 8th IEEE Symposium on Diagnostics for Electrical Machines, Power Electronics and Drives, (1):632-637, 2011.

[82] M. Hernandez-Vargas, E. Cabal-Yepez, A. Garcia-Perez, and R.J. Romero-Troncoso. Novel methodology for broken-rotor-bar and bearing faults detection through SVD and information entropy. Journal of Scientific and Industrial Research, 71(9):589-593, 2012 .

[83] S. Bindu and V.V. Thomas. Diagnoses of internal faults of three phase squirrel cage induction motor - A review. 2014 International Conference on Advances in Energy Conversion Technologies - Intelligent Energy Management: Technologies and Challenges, ICAECT 2014, pages 48-54, 2014.

[84] P. Zhang, Y. Du, T.G. Habetler, and B. Lu. A survey of condition monitoring and protection methods for medium-voltage induction motors. IEEE Transactions on Industry Applications, 47(1):34-46, 2011. 
[85] US Energy Information Administration (USEIA). Today in Energy - Electricity use by machine drives varies significantly by manufacturing industry, 2013.

[86] A. Sadoughi, M. Ebrahimi, and E. Rezaei. A new approach for induction motor broken bar diagnosis by using vibration spectrum. 2006 SICE-ICASE International Joint Conference, pages 4715-4720, 2006.

[87] P.D. McFadden and J.D. Smith. The vibration produced by multiple point defects in a rolling element bearing. Journal of Sound and Vibration, 98(2):263-273, jan 1985.

[88] Z. Kiral and H. Karagülle. Simulation and analysis of vibration signals generated by rolling element bearing with defects. Tribology International, 36(9):667-678, 2003.

[89] W.R. Finley, M.M. Hodowanec, and W.G. Holter. An analytical approach to solving motor vibration problems. IEEE Transactions on Industry Applications, 36(5):217-232, 2000.

[90] M. Salah, K. Bacha, and A. Chaari. Comparative investigation of diagnosis media for induction machine mechanical unbalance fault. ISA Transactions, 52(6):888-899, 2013.

[91] H. P. Bloch. Machinery Failure Analysis and Troubleshooting. Gulf Publishing Company, Houston, Tx, USA, 3rd edition, 1999.

[92] O. Lemoine, P. Flandrin, P. Gonçalves, and A. François. Time-Frequency Toolbox For Use whit MatLab. Technical report, CNRS, Rice University, Paris, France; Houston, Tx, USA, 1996.

[93] F. Hlawatsch and F. Auger. Time-Frequency Analysis. ISTE. Wiley, 2013.

[94] L. Cohen. Time-frequency distributions-a review. Proceedings of the IEEE, 77(7):941981, jul 1989.

[95] L. Cohen. Time-frequency Analysis. Electrical engineering signal processing. Prentice Hall PTR, 1995. 
[96] S.H. Cho, G. Jang, and S.H. Kwon. Time-Frequency Analysis of Power-Quality Disturbances via the Gabor-Wigner Transform. IEEE Transactions on Power Delivery, 25(1):494-499, jan 2010.

[97] P.L. Søndergaard. Efficient Algorithms for the Discrete Gabor Transform with a Long Fir Window. Journal of Fourier Analysis and Applications, 18(3):456-470, 2011.

[98] M.J. Bastiaans and M.C.W. Geilen. On the discrete Gabor transform and the discrete Zak transform. Signal Processing, 49(3):151-166, mar 1996.

[99] S. Mallat. A Wavelet Tour of Signal Processing: The Sparse Way. Elsevier Science, 2008.

[100] M.H. Hayes. Statistical Digital Signal Processing and Modeling. John Wiley \& Sons, Inc., New York, NY, USA, 1st edition, 1996.

[101] N.E. Huang, Z. Shen, S.R. Long, M.C. Wu, H.H. Shih, Q. Zheng, N.C. Yen, C.C. Tung, and H.H. Liu. The empirical mode decomposition and the Hilbert spectrum for nonlinear and non-stationary time series analysis. Proceedings of the Royal Society A: Mathematical, Physical and Engineering Sciences, 454(1971):995, 903, 1998.

[102] P. Flandrin, G. Rilling, and P. Goncalves. Empirical mode decomposition as a filter bank. IEEE Signal Processing Letters, 11(2):112-114, 2004.

[103] Z. Wu and N.E. Huang. Ensemble Empirical Mode Decomposition: A noise-assisted data analysis method. Advances in Adaptive Data Analysis, 01(01):1-41, jan 2009.

[104] M.E. Torres, M.A. Colominas, G. Schlotthauer, and P. Flandrin. A complete ensemble empirical mode decomposition with adaptive noise. In 2011 IEEE International Conference on Acoustics, Speech and Signal Processing (ICASSP), pages 4144-4147. IEEE, may 2011.

[105] A. Garcia-Perez, O. Ibarra-Manzano, and R. J. Romero-Troncoso. Analysis of partially broken rotor bar by using a novel empirical mode decomposition method. IECON 2014 
- 40th Annual Conference of the IEEE Industrial Electronics Society, pages 3403-3408, 2014. 


\section{Glosario y siglas}

\section{Glosario}

Desbalance: El desbalance mecánico se presenta en elementos rotativos cuando el centro geométrico no coincide con el centro de masa.

Eje: Parte del motor de inducción destinado a guiar el movimiento de rotación del rotor.

Estátor: Parte fija de un motor.

Jaula de ardilla: Es la parte que rota, usada comúnmente en un motor de inducción de corriente alterna.

Motor: Máquina que transforma energía eléctrica en energía mecánica por medio de interacciones electromagnéticas.

Rodamiento: Es un elemento mecánico que reduce la fricción entre un eje y las piezas conectadas a éste por medio de una rodadura, que le sirve de apoyo y facilita su desplazamiento.

Rotor: Parte rotativa de un motor.

Variador de frecuencia: Elemento de control para la velocidad de motores eléctricos de c.a. Este dispositivo además recibe otros nombres como variador de velocidad o inversor. 


\section{Siglas}

AI Artificial intelligence

AST Adaptive slope transform

AWT Analitic wavelet transform

ANN Artificial neural network

CEEMD Complete ensemble empirical mode decomposition

c.a. Corriente alterna

c.d. Corriente directa

CWT Continuous wavelet transform

DWT Discrete wavelet transform

EMD Empirical mode decomposition

EEMD Ensemble empirical mode decomposition

FFT Fast Fourier transform

FLC Fuzzy logic controller

FPGA Field programable gate array

HT Hilbert transform

ICA Independent component analysis

IMF Intrinsic mode function

IMFs Intrinsic mode functions

JCR Journal citation report

MCSA Motor current signature analysis

MUSIC Multiple signal classification

PSD Power spectral density

STFT Short-time Fourier transform

TFDG Time-frequency distribution of Gabor

TFMS Time-frequency Morlet scalogram

VFD Variable frequency drive

VSD Variable speed drive

WT Wavelet transform 
WVD Wigner-Ville distribution

ZAM Zhao-Atlas-Marks 